\title{
Central limit theorems for spatial averages of the stochastic heat equation via Malliavin-Stein's method*
}

\author{
Le Chen \\ Emory University \\ le. chen@emory.edu
}

\author{
Davar Khoshnevisan \\ University of Utah \\ davar@math . utah.edu \\ Fei $\mathrm{Pu}$ \\ University of Luxembourg \\ fei.pu@uni.lu
}

\author{
David Nualart \\ University of Kansas \\ nualart@ku.edu
}

August 7, 2020

\begin{abstract}
Suppose that $\{u(t, x)\}_{t>0, x \in \mathbb{R}^{d}}$ is the solution to a $d$-dimensional stochastic heat equation driven by a Gaussian noise that is white in time and has a spatially homogeneous covariance that satisfies Dalang's condition. The purpose of this paper is to establish quantitative central limit theorems for spatial averages of the form $N^{-d} \int_{[0, N]^{d}} g(u(t, x)) \mathrm{d} x$, as $N \rightarrow \infty$, where $g$ is a Lipschitz-continuous function or belongs to a class of locally-Lipschitz functions, using a combination of the Malliavin calculus and Stein's method for normal approximations. Our results include a central limit theorem for the Hopf-Cole solution to KPZ equation. We also establish a functional central limit theorem for these spatial averages.
\end{abstract}

MSC 2010 subject classification: 60H015, 60H07, 60F05.

Keywords: Stochastic heat equation, ergodicity, central limit theorem, Malliavin calculus, Stein's method.

Running head: CLT for SPDEs.

\section{Contents}

1 Introduction $\quad 2$

2 Main results $\quad \mathbf{5}$

${ }^{*}$ Research supported in part by NSF grants DMS-1811181 (D.N.) and DMS-1855439 (D.K.), and FNR grant APOGee (R-AGR-3585-10) at University of Luxembourg (F.P.). 
3.1 Malliavin's calculus and Stein's method . . . . . . . . . . . . . . . . . 10

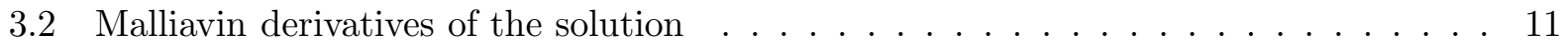

3.3 Malliavin derivatives and shifts of the noise . . . . . . . . . . . . 16

3.4 An abstract ergodicity result . . . . . . . . . . . . . . 17

4 CLT: Proof of Theorem 2.1 20

$5 \quad$ Functional CLT: Proof of Theorem 2.3 26

6 Total Variation Distance for linear $g$ : Proof of Theorem 2.432

7 Parabolic Anderson Model: Proof of Theorem 2.5 36

$\begin{array}{ll}\text { A Association for parabolic SPDEs } & 39\end{array}$

\section{Introduction}

Consider the following stochastic heat equation on $\mathbb{R}^{d}$ :

$$
\frac{\partial u}{\partial t}(t, x)=\frac{1}{2} \Delta u(t, x)+\sigma(u(t, x)) \eta(t, x) \quad \text { for all }(t, x) \in(0, \infty) \times \mathbb{R}^{d},
$$

subject to $u(0, x)=1$ for all $x \in \mathbb{R}^{d}$. The diffusion coefficient $\sigma: \mathbb{R} \rightarrow \mathbb{R}$ is assumed to be nonrandom and Lipschitz continuous. Moreover, we avoid trivialities by always assuming that

$$
\sigma(1) \neq 0 .^{1}
$$

The noise term $\eta$ denotes a centered, generalized Gaussian random field such that

$$
\operatorname{Cov}[\eta(t, x), \eta(s, y)]=\delta_{0}(t-s) f(x-y) \quad \text { for all } s, t \geq 0 \text { and } x, y \in \mathbb{R}^{d},
$$

for a nonnegative-definite tempered Borel measure $f$ on $\mathbb{R}^{d}$ that we fix throughout. More formally, this means that the Wiener-integrals

$$
W_{t}(\phi):=\int_{(0, t) \times \mathbb{R}^{d}} \phi(x) \eta(\mathrm{d} r \mathrm{~d} x) \quad\left[t \geq 0, \phi \in \mathscr{S}\left(\mathbb{R}^{d}\right)\right]
$$

define a centered Gaussian random field with covariance

$$
\operatorname{Cov}\left[W_{s}\left(\phi_{1}\right), W_{t}\left(\phi_{2}\right)\right]=(s \wedge t)\left\langle\phi_{1}, \phi_{2} * f\right\rangle_{L^{2}\left(\mathbb{R}^{d}\right)} \quad \text { for all } s, t \geq 0 \text { and } \phi_{1}, \phi_{2} \in \mathscr{S}\left(\mathbb{R}^{d}\right) .
$$

Note, among other things, that $\left\{W_{t}\right\}_{t \geq 0}$ is an infinite dimensional Brownian motion.

Recall that the Fourier transform $\hat{f}$ of the measure $f$ is a tempered Borel measure on $\mathbb{R}^{d}$. We

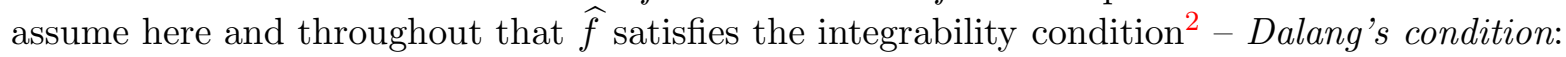

$$
\int_{\mathbb{R}^{d}} \frac{\widehat{f}(\mathrm{~d} z)}{1+\|z\|^{2}}<\infty .
$$

\footnotetext{
${ }^{1}$ Indeed, if $\sigma(1)=0$ then it can be checked that the the solution is degenerate: $u(t, x) \equiv 1$ for all $(t, x) \in \mathbb{R}_{+} \times \mathbb{R}^{d}$.

${ }^{2}$ To be concrete, the Fourier transform is normalized so that $\widehat{h}(z)=\int_{\mathbb{R}^{d}} \mathrm{e}^{i x \cdot z} h(x) \mathrm{d} x$ for all $h \in L^{1}\left(\mathbb{R}^{d}\right)$ and $z \in \mathbb{R}^{d}$.
} 
For some results, we will need the following reinforced version of Dalang's condition:

$$
\int_{\mathbb{R}^{d}} \frac{\widehat{f}(\mathrm{~d} z)}{\left(1+\|z\|^{2}\right)^{1-\alpha}}<\infty, \quad \text { for some } \alpha \in(0,1]
$$

Dalang [15] has proved that, because of (1.5), the stochastic heat equation (1.1) has a mild solution $u=\{u(t, x)\}_{t \geq 0, x \in \mathbb{R}^{d}}$ that is the unique predictable random field such that

$$
\sup _{t \in[0, T]} \sup _{x \in \mathbb{R}^{d}} \mathrm{E}\left(|u(t, x)|^{k}\right)<\infty \quad \text { for every } T>0 \text { and } k \geq 2 .
$$

Moreover, "mild" refers to the fact that $u$ satisfies the evolution equation

$$
u(t, x)=1+\int_{(0, t) \times \mathbb{R}^{d}} \boldsymbol{p}_{t-s}(x-y) \sigma(u(s, y)) \eta(\mathrm{d} s \mathrm{~d} y) \quad \text { for all } t>0 \text { and } x \in \mathbb{R}^{d},
$$

where $\boldsymbol{p}$ denotes the heat kernel on $(0, \infty) \times \mathbb{R}^{d}$; that is,

$$
\boldsymbol{p}_{t}(x)=\frac{1}{(2 \pi t)^{d / 2}} \exp \left(-\frac{\|x\|^{2}}{2 t}\right) \quad\left[t>0, x \in \mathbb{R}^{d}\right]
$$

The mapping $(t, x) \mapsto u(t, x)$ is continuous in $L^{k}(\Omega)$ on $[0, \infty) \times \mathbb{R}^{d}$ for every $k \geq 2$; see Walsh [38] for the case that $f=\delta_{0}$, and Dalang [15] and its method for the general case.

In Ref. [7] we observed that the spatial random field $u(t)=\{u(t, x)\}_{x \in \mathbb{R}^{d}}$ is stationary for every $t \geq 0$, and we proved that $u(t)$ is an ergodic random field for every $t>0$ provided that $\hat{f}\{0\}=0$. In particular, if the spectral measure $\widehat{f}$ does not have an atom at 0 , then the ergodic theorem implies that, for all $t>0$ and all measurable functions $g: \mathbb{R} \rightarrow \mathbb{R}$ such that $\mathrm{E}[|g(u(t, 0))|]<\infty$,

$$
\lim _{N \rightarrow \infty} \frac{1}{N^{d}} \int_{[0, N]^{d}} g(u(t, x)) \mathrm{d} x=\mathrm{E}[g(u(t, 0))] \quad \text { a.s.. }
$$

A natural question is to determine whether (1.9) has a matching central limit theorem (CLT). That is, we would like to establish the convergence in distribution of $N^{d / 2} \mathcal{S}_{N, t}(g)$ to a normal law as $N$ tends to infinity, where

$$
\mathcal{S}_{N, t}(g)=N^{-d} \int_{[0, N]^{d}} g(u(t, x)) \mathrm{d} x-\mathrm{E}[g(u(t, 0))] \quad[N, t>0] .
$$

In a recent paper [8], we have derived such a CLT under the following assumption:

$$
0<f\left(\mathbb{R}^{d}\right)<\infty
$$

when $g \in$ Lip, where "Lip" denotes the collection of all real-valued Lipschitz-continuous functions on $\mathbb{R}$. Our proof rested on Poincaré-type inequalities and Malliavin's calculus, as well as compactness arguments and Lévy's characterization of Brownian motion. The positivity of the total mass of $f$ [in (1.11)] merely ensures nontriviality. And the finite-mass condition on $f$ turns out to be optimal. Moreover, we considered, instead of only CLTs for $N^{-d} \int_{[0, N]} g(u(t, x)) \mathrm{d} x$, functional CLTs for $N^{-d} \int_{\mathbb{R}^{d}} \psi(x / N) g(u(t, x)) \mathrm{d} x$ where $\psi: \mathbb{R}^{d} \rightarrow \mathbb{R}$ ranges over a large class of nice functions.

In the present paper we appeal to the Malliavin-Stein method in order to establish convergence of $N^{d / 2} \mathcal{S}_{N, t}(g)$ to a normal law in the total variation distance, which we denote by $d_{\mathrm{TV}}$ throughout. As was initiated by Nourdin and Peccati [30,31], the combination of Malliavin's calculus with Stein's 
method for normal approximations can provide an effective method for deriving quantitative CLTs for functionals of Gaussian random fields. Moreover, we will establish such central limit theorems not only for $g \in$ Lip, but also for certain locally Lipschitz functions such as

$$
g(u)=\log (u) \text { and } g(u)=u^{\alpha} \quad \text { for } \alpha \neq 0 \text { and } u>0 .
$$

In particular, our results will cover the case when $g(u)=\log (u)\left(\right.$ with $\sigma(u)=u, f=\delta_{0}, d=1$ ), in which case $g(u)$ is called the Hopf-Cole solution or height function of the corresponding KardarParisi-Zhang (KPZ) equation [26].

Huang et al [24] were the first to use the Malliavin-Stein method in order to study spatial averages of parabolic SPDEs. They (ibid.) studied the case that $d=1$ and $f=\delta_{0}$ - that is, the stochastic heat equation in $1+1$ dimension, driven by space-time white noise - and proved that, in the case that $g(u)=u$,

$$
d_{\mathrm{TV}}\left(N^{1 / 2} \mathcal{S}_{N, t}(u), X\right)=O(1 / \sqrt{N}) \quad \text { as } N \rightarrow \infty,
$$

for every fixed $t>0$, where $X=X(t)$ has a centered normal distribution. Huang et al [23,25] study the case that $d \geq 1$ and $f(\mathrm{~d} x)=\|x\|^{-\beta} \mathrm{d} x$ for some $\beta \in(0, d \wedge 2)$. This Riesz-type covariance form does not satisfy condition (1.11). Huang et al (ibid.) establish a CLT though with a nonstandard normalization that depends on the numerical value of the Riesz kernel index $\beta$. More recently, Nualart and Zheng [34] use Wiener-chaos expansions to derive CLTs in the case that $\sigma(u)=u$ and $g(u)=u$.

Let us next describe some of the highlights of this paper. Precise formulations can be found in the next section.

Theorem 2.1 states that if the variance of $N^{d / 2} \mathcal{S}_{N, t}(g)$ converges to a strictly positive real number, then $N^{d / 2} \mathcal{S}_{N, t}(g)$ converges in total variation to a normal law. The proof is based on the Malliavin-Stein approach, as well as on an abstract ergodic theorem for functionals of the underlying Gaussian noise $\eta$. Sufficient conditions for the limit variance to be nonzero are given in Proposition 2.6.

Theorem 2.3 provides a functional version, in the time variable, of some of our recent work [8, Theorem 1.1]. This result is a non-trivial extension of a functional CLT of Huang et al. in [24], valid for SPDEs that are driven by space-time white noise.

We are able to explore the rate of convergence in total variation of $N^{d / 2} \mathcal{S}_{N, t}(g)$ to a normal law in two special cases:

1. In Theorem 2.4 we study the case that $g(v)=v$ for all $v \in \mathbb{R}$, and find bounds on the total variation distance between $\mathcal{S}_{N, t}(g)$, normalized by its standard deviation, and $\mathrm{N}(0,1)$; and

2. In Theorem 2.5 we restrict our attention to a general $g$, but study the rate-of-convergence problem for the so-called parabolic Anderson model, which is (1.1) in the case that $\sigma(z)=z$ for all $z \in \mathbb{R}$.

In Theorems 2.1, 2.3 and 2.5, $g$ can be either a globally Lipschitz function or certain locally Lipschitz function that includes examples in (1.12). The proofs of these theorems can be found respectively in Sections 4, 5, 6 and 7.

In this paper and its companion papers [7-9] we have presented several new ways of establishing CLTs for functionals of infinitely-many particle systems based on Malliavin's calculus, compactness, Poincaré inequalities, the Malliavin-Stein method, and Clark-Ocone formulas. Previous, more established methods, for deriving CLTs for particle systems include:

- The use of martingale CLTs (see, for example, Deuschel [16]);

- CLTs for strongly mixing processes (see, for example, the monograph of Bradley [1]); and 
- Techniques based on association, a notion that we recall in the appendix (see, for example, Newman and Wright [29] with various extensions that can be found in Newman [28], and Rao [36]).

We can compare the techniques of this paper and its companions (ibid.) with the above methods in the context of SPDEs and systems of interacting SDEs as follows:

- Our methods provide significant improvements over those that appeal to martingale CLTs (see [9], and consider adapting the methods of the present paper to the semi-discrete setting [16] for still dramatic improvements such as those hinted at in Corollary 2.7 below);

- We do not know how to establish strong mixing for SPDEs except possibly when $\sigma$ is constant. In that very simple case, the solution to (1.1) is a Gaussian process and one can try to adapt the existing ergodic theory (see for example Helson and Sarason [22], and Dym and McKean [17] for an overview) to the present setting. We warn however that even that effort will likely require a fair amount of work; and

- We can try to implement association techniques to derive CLTs for SPDEs (instead of the present methods). It is known that the solution to (1.1) is associated in a few special instances: Corwin and Quastel [14] (see also Corwin and Ghosal [13]) observed that $u$ is associated when $d=1, f=\delta_{0}$, and $\sigma(u)=u$; and a theorem of Pitt [35] implies that $u$ is associated whenever $\sigma$ is a constant. The strongest association result that we are able to prove requires a good deal more effort, and yet only states that: $u$ is associated provided that $\sigma(u)$ does not change sign; see Theorem A.4. As we shall see, CLTs for (1.1) do not require that $\sigma(u)$ does not change sign. Therefore, we will not pursue association ideas vigorously here.

Throughout, $\mathcal{F}=\left\{\mathcal{F}_{t}\right\}_{t \geq 0}$ denotes the Brownian filtration generated by the infinite dimensional Brownian motion $\left\{W_{t}\right\}_{t \geq 0}$, defined in (1.4). As is customary, we assume that $\mathcal{F}$ is augmented in the usual way. For every $Z \in L^{k}(\Omega)$, we write $\|Z\|_{k}$ instead of the more cumbersome $\|Z\|_{L^{k}(\Omega)}=$ $\left\{\mathrm{E}\left(|Z|^{k}\right)\right\}^{1 / k}$. We use $\mathrm{N}(0,1)$ to denote the standard normal distribution. For every $g: \mathbb{R} \mapsto \mathbb{R}$ we write

$$
\operatorname{Lip}(g)=\sup _{-\infty<a<b<\infty} \frac{|g(b)-g(a)|}{|b-a|} .
$$

Thus, $g \in$ Lip if and only if $\operatorname{Lip}(g)<\infty$.

\section{Main results}

Let $\mathcal{S}_{N, t}(g)$ denote the random variable defined in (1.10) for every $t \geq 0, N>0$, and any measurable function $g: \mathbb{R} \rightarrow \mathbb{R}$. Throughout, we will write, for $t_{1}, t_{2} \geq 0$,

$$
\mathbf{B}_{N, t_{1}, t_{2}}(g):=\operatorname{Cov}\left(N^{d / 2} \mathcal{S}_{N, t_{1}}(g), N^{d / 2} \mathcal{S}_{N, t_{2}}(g)\right)
$$

and for $t_{1}=t_{2}=t$ we put $\mathbf{B}_{N, t}(g)=\mathbf{B}_{N, t, t}(g)$. We have proven in [8, Proposition 5.2 $]^{3}$ that, when $g \in \operatorname{Lip}$,

$$
\lim _{N \rightarrow \infty} \mathbf{B}_{N, t_{1}, t_{2}}(g)=\mathbf{B}_{t_{1}, t_{2}}(g):=\int_{\mathbb{R}^{d}} \operatorname{Cov}\left[g\left(u\left(t_{1}, x\right)\right), g\left(u\left(t_{2}, 0\right)\right)\right] \mathrm{d} x,
$$

and

$$
\int_{\mathbb{R}^{d}}\left|\operatorname{Cov}\left[g\left(u\left(t_{1}, x\right)\right), g\left(u\left(t_{2}, 0\right)\right)\right]\right| \mathrm{d} x<\infty
$$

see [8, Lemma 5.1]. Denote $\mathbf{B}_{t}(g)=\mathbf{B}_{t, t}(g)$.

\footnotetext{
${ }^{3}$ This result is proved in [8] in the case $t_{1}=t_{2}$ and the proof in the case $t_{1} \neq t_{2}$ is analogous.
} 
We are also interested in the cases when the above Lipschitz functions $g$ are replaced by certain locally Lipschitz ones. In particular, assuming $\sigma(0)=0$, the solution $u(t, x)$ is strictly positive almost surely and it turns out that the following set of conditions will be sufficient for this purpose:

$$
\left\{\begin{array}{l}
g \in C^{2}(0, \infty), \\
g^{\prime} \text { is either strictly positive or strictly negative, } \\
g^{\prime \prime} \text { is monotone over }(0, \infty), \\
\mathrm{E}\left(\left|\frac{\mathrm{d}^{i} g}{\mathrm{~d} x^{i}}(u(t, 0))\right|^{k}\right)<\infty, \quad \text { for all } t>0, i \in\{0,1,2\} \text { and } k \in \mathbb{Z} .
\end{array}\right.
$$

It is clear that conditions (2.4a) - (2.4c) are satisfied by examples in (1.12). As for condition (2.4d), thanks to the comparison principle established by Chen and Huang [5] and nonnegative moments proved in Chen and Huang [6, Theorem 1.8] (see also Theorem 5.1 of Conus et al [11] for the case when $d=1$ and $f=\delta_{0}$ ), by assuming both condition $\sigma(0)=0$ and the reinforced Dalang's condition (1.6), all examples in (1.12) satisfy condition (2.4d) (even for all $i \geq 0$ ). One can see that conditions regarding locally Lipschitz $g$ in Theorems 2.1, 2.3, and 2.5 are all special cases of (2.4).

Now we are ready to state our results. The following is the first result of this paper, and shows that the weak convergence theorem of [8, Theorem 1.1] holds in total variation when the limit variance is not zero. This is a strict improvement because manifestly $\lim _{N \rightarrow \infty} N^{d / 2} \mathcal{S}_{N, t}(g)=0$ in $L^{2}(\Omega)$ when the limiting variance is zero.

Theorem 2.1. Let $u$ denote the solution to (1.1) with $u(0) \equiv 1$ and $\sigma(1) \neq 0$ and suppose that (1.11) holds. Suppose that one of the three conditions holds:

(i) $g \in \operatorname{Lip}$,

(ii) $g \in C^{1}(\mathbb{R})$ and for some $k>4$

$$
\mathrm{E}\left(\left|g^{(i)}(u(s, 0))\right|^{k}\right)<\infty, \quad \text { for all } s>0, i \in\{0,1\}
$$

(iii) $\sigma(0)=0, f$ satisfies the reinforced Dalang's condition (1.6), $g \in C^{1}(0, \infty)$ and (2.5) holds.

Then, properties (2.2) and (2.3) are satisfied and, for any $t>0$, provided that $\mathbf{B}_{t}(g)>0$, we have that

$$
\lim _{N \rightarrow \infty} d_{\mathrm{TV}}\left(N^{d / 2} \mathcal{S}_{N, t}(g), \sqrt{\mathbf{B}_{t}(g)} \mathrm{N}(0,1)\right)=0 .
$$

Moreover, in Case (iii), we have the following sufficient condition for $\mathbf{B}_{t}(g) \in(0, \infty)$ :

$$
\sigma(x) \neq 0 \text { for all } x>0 \text { and } g^{\prime} \text { is either strictly positive or strictly negative for all } x>0 \text {, }
$$

It is clear that the parabolic Anderson model $\left(d=1, \sigma(u)=u, f=\delta_{0}\right)$ and the function $g(u)=\log u$ satisfy the conditions (iii) and (2.7) of Theorem 2.1; see [11, Theorem 5.1] for the moment condition (2.5). Therefore, Theorem 2.1 implies the following central limit theorem for the Hopf-Cole solution to the KPZ equation.

Corollary 2.2. Assume $d=1, f=\delta_{0}$ and $\sigma(u)=u$ for all $u \in \mathbb{R}$. Then, for all $t>0$,

$$
\frac{1}{\sqrt{N}} \int_{0}^{N}\{\log u(t, x)-\mathrm{E}[\log u(t, 0)]\} \mathrm{d} x \rightarrow \mathrm{N}\left(0, \sigma_{t}^{2}\right) \quad \text { in distribution as } N \rightarrow \infty,
$$

where $\sigma_{t}^{2}:=\int_{-\infty}^{\infty} \operatorname{Cov}[\log u(t, x), \log u(t, 0)] \mathrm{d} x \in(0, \infty)$. 
Our next result provides a functional version in time of the weak convergence proved in $[8$, Theorem 1.1].

Theorem 2.3. Let $u$ denote the solution to (1.1) with $u(0) \equiv 1$ and $\sigma(1) \neq 0$ and suppose that $f$ satisfies both condition (1.11) and the reinforced Dalang's condition (1.6) for some $\alpha \in(0,1]$. Then we have the following two cases:

(i) For any $g \in C^{1}(\mathbb{R})$ such that $g^{\prime}$ is Hölder continuous of order $\delta \in(0,1]$, it holds that

$$
\left\{N^{d / 2} \mathcal{S}_{N, t}(g)\right\}_{t \in[0, T]} \stackrel{C[0, T]}{\longrightarrow}\left\{\mathcal{G}_{t}\right\}_{t \in[0, T]} \quad \text { as } N \rightarrow \infty, \text { for any } T>0,
$$

where $\left\{\mathcal{G}_{t}\right\}_{t \geq 0}$ is a centered Gaussian process with covariance $\mathrm{E}\left[\mathcal{G}_{t_{1}} \mathcal{G}_{t_{2}}\right]=\mathbf{B}_{t_{1}, t_{2}}(g)[$ see (2.2)].

(ii) If $\sigma(0)=0$, then (2.9) holds for any $g \in C^{2}(0, \infty)$ such that $g^{\prime \prime}$ is monotone over $(0, \infty)$ and

$$
\mathrm{E}\left(\left|\frac{\mathrm{d}^{i} g}{\mathrm{~d} x^{i}}(u(t, 0))\right|^{k}\right)<\infty \quad \text { for all } t \geq 0, k \geq 2 \text {, and } i \in\{0,1,2\} \text {. }
$$

In the special case that $g(v)=v$ for all $v \in \mathbb{R}$, we can use Malliavin-Stein's method to show that the total variation distance between $\mathcal{S}_{N, t}(g)$ and a suitable normal distribution is $O\left(N^{-d / 2}\right)$. The next theorem contains the exact form of this statement, and generalizes the recent work of Huang et al [24].

Theorem 2.4. If $g(v)=v$ for all $v \in \mathbb{R}$, then for two real numbers $\lambda, L>0$ - depending only on $(f, \sigma)$ - it holds that

$$
d_{\mathrm{TV}}\left(\frac{\mathcal{S}_{N, t}(g)}{\sqrt{\operatorname{Var}\left(\mathcal{S}_{N, t}(g)\right)}}, \mathrm{N}(0,1)\right) \leq \frac{L \mathrm{e}^{\lambda t}}{N^{d / 2} \mathbf{B}_{N, t}(g)},
$$

uniformly for all $t>0$ such that $\mathbf{B}_{t}(g)>0$ and all $N>0$ large enough to ensure that $\mathbf{B}_{N, t}(g)>0$.

We are able to study the parabolic Anderson model as well. That is the when $\sigma(z)=z$ for all $z \in \mathbb{R}$. Though we hasten to add that the proof of the following is different from that of Theorem 2.4 .

At this point, we are not able to analyze the case where both $\sigma$ and $g$ are fairly general [nice] functions.

Theorem 2.5. Let $u$ denote the solution to (1.1) with $\sigma(z)=z$ and $u(0) \equiv 1$ and assume that $f$ satisfies both (1.11) and the reinforced Dalang's condition (1.6) for some $\alpha \in(0,1]$. Suppose that $g \in C^{2}(0, \infty)$ and for some $k>4$,

$$
\mathrm{E}\left(\left|\frac{\mathrm{d}^{i} g}{\mathrm{~d} x^{i}}(u(t, 0))\right|^{k}\right)<\infty \quad \text { for all } t \geq 0 \text { and } i \in\{0,1,2\} .
$$

Then for two real numbers $\lambda, L>0$ - depending only on $(f, \sigma)$ - it holds that

$$
d_{\mathrm{TV}}\left(\frac{\mathcal{S}_{N, t}(g)}{\sqrt{\operatorname{Var}\left(\mathcal{S}_{N, t}(g)\right)}}, \mathrm{N}(0,1)\right) \leq \frac{L \Theta_{t} \mathrm{e}^{\lambda t}}{N^{d / 2} \mathbf{B}_{N, t}(g)},
$$

uniformly for all $t>0$ such that $\mathbf{B}_{N}(g)>0$ and all $N>0$ large enough to ensure that $\mathbf{B}_{N, t}(g)>0$, where $\Theta_{t}:=\left\|g^{\prime}(u(t, 0))\right\|_{k} \max \left(\left\|g^{\prime}(u(t, 0))\right\|_{k},\left\|g^{\prime \prime}(u(t, 0))\right\|_{k}\right)$. 
In Theorem 2.5, the reinforced Dalang's condition (1.6) is used to ensure that almost surely $u(t, x)>0$ for all $t \geq 0$ and $x \in \mathbb{R}^{d}$. We do not need this condition if we assume $g \in C^{2}(\mathbb{R})$ instead of $g \in C^{2}(0, \infty)$.

Note that both (2.11) and (2.13) are trivial when $\mathbf{B}_{N, t}(g)=0$.

Among other things, Theorems 2.4 and/or 2.5 and Eq.'s (2.1) and (2.2) together imply the following extension of (1.13) from the case that $d=1$ and $f=\delta_{0}$ to the more general settings of Theorems 2.4 and/or 2.5: If either $g(v)=v$ for all $v \in \mathbb{R}^{d}$ or $\sigma(z)=z$ for all $z \in \mathbb{R}$, and if $\mathbf{B}_{t}(g)>0$ then

$$
d_{\mathrm{TV}}\left(\frac{\mathcal{S}_{N, t}(g)}{\sqrt{\operatorname{Var}\left(\mathcal{S}_{N, t}(g)\right)}}, \mathrm{N}(0,1)\right)=O\left(N^{-d / 2}\right) \quad \text { as } N \rightarrow \infty .
$$

Moreover, because Theorem 2.4 and 2.5 involve quantitative bounds on the total variation distance, we can also sometimes use them to prove that $\mathcal{S}_{N, t}(g)$ is asymptotically normal as $N, t \rightarrow \infty$ simultaneously. The following provides the requisite technical result that allows for this sort of undertaking. Before we state the result, let us note that the parabolic Anderson model [that is, $\sigma(z)=z$ for all $z \in \mathbb{R}]$ satisfies all three conditions of the following.

Proposition 2.6. Suppose $g(v)=v$ for all $v \in \mathbb{R}$, and let $\left\{t_{N}\right\}_{N>0}$ be a net of strictly positive numbers such that $t_{N}=o\left(N^{2}\right)$ as $N \rightarrow \infty$. Recall condition (1.2) and suppose additionally that either $Q=\sigma$ or $Q=-\sigma$ satisfy any one of the following conditions:

1. $Q(x) \geq 0$ for all $x>0$;

2. There exists $c>0$ such that $Q(w) \geq c$ for all $w>0$;

3. $Q(0)=0$ and there exists $c>0$ such that $Q(w) \geq c w$ for all $w>0$.

Then, $\liminf _{N \rightarrow \infty} t_{N}^{-1} \mathbf{B}_{N, t_{N}}(g)>0$ under conditions 1 and 2, and $\liminf _{N \rightarrow \infty} \mathbf{B}_{N, t_{N}}(g)>0$ under condition 3.

Indeed, Theorems 2.4 and 2.5, together with Proposition 2.6, yield the following result immediately, with no need for additional justification:

Corollary 2.7. Suppose that $g(v)=v$ for all $v \in \mathbb{R}, \sigma$ satisfies one of the three conditions of Proposition 2.6, and $\left\{t_{N}\right\}_{N>0}$ is a net of strictly positive numbers. Then,

$$
\lim _{N \rightarrow \infty} d_{\mathrm{TV}}\left(\frac{\mathcal{S}_{N, t_{N}}(g)}{\sqrt{\operatorname{Var}\left(\mathcal{S}_{N, t_{N}}(g)\right)}}, \mathrm{N}(0,1)\right)=0 \text { provided that } t_{N}=o(\log N) \quad \text { as } N \rightarrow \infty .
$$

Such time-dependent CLTs have been anticipated by Deuschel [16].

In light of our earlier work [8], the proof of Proposition 2.6 is short enough, and simple enough, that it can be included here.

Proof of Proposition 2.6. According to the proof of Proposition 5.3 of Chen et al [8], there exists a real number $C>0$ such that, under either condition 1 or condition 2 ,

$$
\operatorname{Cov}[u(t, x), u(t, y)] \geq C \int_{0}^{t}\left(\boldsymbol{p}_{2 s} * f\right)(x-y) \mathrm{d} s \quad \text { for all } t>0 \text { and } x, y \in \mathbb{R}^{d} .
$$


Since $g(v)=v$ for all $v \in \mathbb{R}$, it follows that

$$
\begin{aligned}
\mathbf{B}_{N, t}(g) & \geq \frac{C}{N^{d}} \int_{[0, N]^{d}} \mathrm{~d} x \int_{[0, N]^{d}} \mathrm{~d} y \int_{0}^{t} \mathrm{~d} s\left(\boldsymbol{p}_{2 s} * f\right)(x-y) \\
& =\frac{C}{N^{d}} \int_{[0, N]^{d}} \mathrm{~d} x \int_{x-[0, N]^{d}} \mathrm{~d} z \int_{0}^{t} \mathrm{~d} s\left(\boldsymbol{p}_{2 s} * f\right)(z) \\
& \geq \frac{C}{N^{d}} \int_{[N / 4,3 N / 4]^{d}} \mathrm{~d} x \int_{[-N / 4, N / 4]^{d}} \mathrm{~d} z \int_{0}^{t} \mathrm{~d} s\left(\boldsymbol{p}_{2 s} * f\right)(z) \\
& =\frac{C}{2^{d}} \int_{[-N / 4, N / 4]^{d}} \mathrm{~d} z \int_{0}^{t} \mathrm{~d} s\left(\boldsymbol{p}_{2 s} * f\right)(z) \\
& =\frac{C}{2^{d}} t f\left(\mathbb{R}^{d}\right)-\frac{C}{2^{d}} \int_{\mathbb{R}^{d} \backslash[-N / 4, N / 4]^{d}} \mathrm{~d} z \int_{0}^{t} \mathrm{~d} s\left(\boldsymbol{p}_{2 s} * f\right)(z) .
\end{aligned}
$$

If $z \notin[-N / 4, N / 4]^{d}$ and $y \in[-N / 8, N / 8]^{d}$, then $z-y \notin[-N / 8, N / 8]^{d}$, and hence

$$
\begin{aligned}
& \int_{\mathbb{R}^{d} \backslash[-N / 4, N / 4]^{d}} \mathrm{~d} z \int_{0}^{t} \mathrm{~d} s\left(\boldsymbol{p}_{2 s} * f\right)(z) \\
& \quad \leq f\left([-N / 8, N / 8]^{d}\right) \int_{\mathbb{R}^{d} \backslash[-N / 8, N / 8]^{d}} \mathrm{~d} x \int_{0}^{t} \mathrm{~d} s \boldsymbol{p}_{2 s}(x)+t f\left(\mathbb{R}^{d} \backslash[-N / 8, N / 8]^{d}\right) \\
& \quad \leq t f\left(\mathbb{R}^{d}\right) \int_{\left.\mathbb{R}^{d} \backslash[-N /(8 \sqrt{t})], N /(8 \sqrt{t})\right]^{d}} \boldsymbol{p}_{2}(x) \mathrm{d} x+t f\left(\mathbb{R}^{d} \backslash[-N / 8, N / 8]^{d}\right) .
\end{aligned}
$$

Replace $t$ by $t_{N}$ and use the fact that $t_{N}=o\left(N^{2}\right)$ as $N \rightarrow \infty$ in order to complete the proof of the proposition under conditions 1 and/or 2 .

Next suppose condition 3 of the proposition holds. In this case, the proof of Proposition 5.3 of Chen et al [8] yields strictly positive numbers $\delta$ and $R$ such that

$\operatorname{Cov}[u(t, x), u(t, y)] \geq \frac{[\sigma(1)]^{2}}{2} \int_{0}^{\delta} \mathrm{d} s \int_{[-R, R]^{d}} f(\mathrm{~d} w) \boldsymbol{p}_{2(t-s)}(x-y+w) \quad$ for all $t>0$ and $x, y \in \mathbb{R}^{d}$.

Therefore,

$$
\begin{aligned}
\mathbf{B}_{N, t}(g) & \geq \frac{[\sigma(1)]^{2}}{2 N^{d}} \int_{0}^{\delta} \mathrm{d} s \int_{[-R, R]^{d}} f(\mathrm{~d} w) \int_{[0, N]^{d}} \mathrm{~d} x \int_{[0, N]^{d}} \mathrm{~d} y \boldsymbol{p}_{2(t-s)}(x-y+w) \\
& =\frac{[\sigma(1)]^{2}}{2 N^{d}} \int_{t-\delta}^{t} \mathrm{~d} s \int_{[-R, R]^{d}} f(\mathrm{~d} w) \int_{[0, N]^{d}} \mathrm{~d} x \int_{x-[0, N]^{d}} \mathrm{~d} z \boldsymbol{p}_{2 s}(z+w) \\
& \geq \frac{[\sigma(1)]^{2}}{2^{d+1}} \int_{t-\delta}^{t} \mathrm{~d} s \int_{[-R, R]^{d}} f(\mathrm{~d} w) \int_{[-N / 4, N / 4]^{d}} \mathrm{~d} z \boldsymbol{p}_{2 s}(z+w) \\
& =\frac{[\sigma(1)]^{2} \delta}{2^{d+1}} f\left([-R, R]^{d}\right)-\frac{[\sigma(1)]^{2}}{2^{d+1}} \int_{t-\delta}^{t} \mathrm{~d} s \int_{[-R, R]^{d}} f(\mathrm{~d} w) \int_{\mathbb{R}^{d} \backslash[-N / 4, N / 4]^{d}} \mathrm{~d} z \boldsymbol{p}_{2 s}(z+w) .
\end{aligned}
$$

The preceding triple integral can be bounded from above as follows:

$$
\begin{aligned}
\int_{t-\delta}^{t} \mathrm{~d} s \int_{[-R, R]^{d}} f(\mathrm{~d} w) \int_{\mathbb{R}^{d} \backslash[-N / 4, N / 4]^{d}} \mathrm{~d} z \boldsymbol{p}_{2 s}(z+w) & \leq f\left(\mathbb{R}^{d}\right) \int_{t-\delta}^{t} \mathrm{~d} s \int_{\mathbb{R}^{d} \backslash[R-N / 4,-R+N / 4]^{d}} \mathrm{~d} x \boldsymbol{p}_{2 s}(x) \\
& \leq f\left(\mathbb{R}^{d}\right) \delta \int_{\mathbb{R}^{d} \backslash[(R-N / 4) / \sqrt{t},(N / 4-R) / \sqrt{t}]^{d}} \boldsymbol{p}_{2}(x) \mathrm{d} x .
\end{aligned}
$$

Replace $t$ by $t_{N}$ and observe that the integral tends to zero as $N \rightarrow \infty$ because $t_{N}=o\left(N^{2}\right)$ as $N \rightarrow \infty$. This completes the proof because $f\left([-R, R]^{d}\right)>0$; see Chen et al [8, Proposition 5.3]. 


\section{Preliminaries}

In this section we collect some basic facts about the Malliavin-Stein method. We also derive estimates on the $L^{k}(\Omega)$-norm of the Malliavin derivatives of the solution $u=\{u(t, x)\}_{t \geq 0, x \in \mathbb{R}^{d}}$ to the stochastic PDE (1.1). These $L^{k}(\Omega)$ estimates will play a pivotal role in the remainder of the paper. We also provide sufficient conditions, based on our earlier work [8], for the nondegeneracy of the limiting variance of $N^{d / 2} \mathcal{S}_{N, t}(g)$.

\subsection{Malliavin's calculus and Stein's method}

Let $\mathcal{H}_{0}$ denote the Hilbert space that is obtained by completing $\mathscr{S}\left(\mathbb{R}^{d}\right)$ under the pre-Hilbertian norm that is defined by scalar product $\left\langle\phi_{1}, \phi_{2}\right\rangle_{\mathcal{H}_{0}}=\left\langle\phi_{1}, \phi_{2} * f\right\rangle_{L^{2}\left(\mathbb{R}^{d}\right)}$, and let $\mathcal{H}=L^{2}\left(\mathbb{R}_{+} ; \mathcal{H}_{0}\right)$. The Gaussian random field $\{\eta(h)\}_{\phi \in \mathcal{H}}$, formed by the Wiener integrals

$$
\eta(h):=\int_{\mathbb{R}_{+} \times \mathbb{R}^{d}} h(s, x) \eta(\mathrm{d} s \mathrm{~d} x) \quad[h \in \mathcal{H}],
$$

is called the isonormal Gaussian process on the Hilbert space $\mathcal{H}$. Thus, we can develop the Malliavin calculus in this framework; see, for instance, Nualart [32].

We denote by $D$ the Malliavin derivative operator and by $\delta$ the corresponding divergence operator that is defined by the following adjoint relation:

$$
\mathrm{E}\left(\langle D F, v\rangle_{\mathcal{H}}\right)=\mathrm{E}[F \delta(v)],
$$

valid for every random variable $F$ in the Gaussian Sobolev space $\mathbb{D}^{1,2}$ and every $v$ in the domain in $L^{2}(\Omega)$ of $\delta$. An important property of the divergence operator is that

$$
\delta(F)=\int_{\mathbb{R}_{+} \times \mathbb{R}^{d}} F(s, x) \eta(\mathrm{d} s \mathrm{~d} x) \quad[\text { the Walsh integral }]
$$

when $F$ is a predictable and square-integrable random field.

We make extensive use of the following form of the Clark-Ocone formula (see Chen et al [7, Proposition 6.3]):

$$
F=\mathrm{E} F+\int_{\mathbb{R}_{+} \times \mathbb{R}^{d}} \mathrm{E}\left(D_{s, z} F \mid \mathcal{F}_{s}\right) \eta(\mathrm{d} s \mathrm{~d} z) \quad \text { a.s. for every } F \in \mathbb{D}^{1,2} .
$$

Among other things, the Clark-Ocone formula readily yields the Poincaré inequality,

$$
\operatorname{Var}(F) \leq \mathrm{E}\left(\|D F\|_{\mathcal{H}}^{2}\right) \quad \text { for all } F \in \mathbb{D}^{1,2} .
$$

Recall that the total variation distance between two probability measures $\mu$ and $\nu$ on $\mathbb{R}$ is defined by

$$
d_{\mathrm{TV}}(\mu, \nu)=\sup _{B \in \mathcal{B}(\mathbb{R})}|\mu(B)-\nu(B)|,
$$

where $\mathcal{B}(\mathbb{R})$ denotes the family of all Borel subsets on $\mathbb{R}$. As is customary, we let $d_{\mathrm{TV}}(F, G)$ denote the total variation distance between the laws of $F$ and $G$ whenever $F$ and $G$ are random variables. And $d_{\mathrm{TV}}(F, \mathrm{~N}(a, b))$ is written interchangeably for $d_{\mathrm{TV}}(F, G)$ where $G$ has a normal distribution with mean $a$ and variance $b$.

The combination of Stein's method for normal approximations with Malliavin calculus leads to the following bound on the total variation distance. See Nualart and Nualart [33, Theorem 8.2.1] for details and proof. 
Proposition 3.1. Suppose that $F \in \mathbb{D}^{1,2}$ satisfies $F=\delta(v)$ for some $v$ in the domain in $L^{2}(\Omega)$ of the divergence operator $\delta$, and suppose that $\tau^{2}:=\mathrm{E}\left(F^{2}\right)>0$. Then

$$
d_{\mathrm{TV}}\left(F, \mathrm{~N}\left(0, \tau^{2}\right)\right) \leq \frac{2}{\tau^{2}} \mathrm{E}\left(\left|\tau^{2}-\langle D F, v\rangle_{\mathcal{H}}\right|\right) .
$$

Thanks to the duality relationship (3.2), the pair $(F, v)$ of Proposition 3.1 satisfies

$$
\mathrm{E}\left(\langle D F, v\rangle_{\mathcal{H}}\right)=\tau^{2}=\operatorname{Var}(F)
$$

Therefore, Proposition 3.1 is bounding the total variation distance between $F$ and $\mathrm{N}(0, \operatorname{Var}(F))$ by the centralized $L^{1}$-norm of $\langle D F, v\rangle_{\mathcal{H}}$. Note also that, as a consequence of (3.5), (3.6), and Jensen's inequality,

$$
d_{\mathrm{TV}}(F, \mathrm{~N}(0,1)) \leq 2 \sqrt{\operatorname{Var}\left(\langle D F, v\rangle_{\mathcal{H}}\right)} \quad \text { when } \operatorname{Var}(F)=1
$$

\subsection{Malliavin derivatives of the solution}

In this part, we will establish the strict positivity of the Malliavin derivatives to the stochastic heat equation in Theorem 3.2 and derive its moment estimates in Lemma 3.3 and Proposition 3.4. We first establish the positivity of the Malliavin derivative and it will be a key ingredient in the proof of the positivity of the limiting variance in our CLT.

Theorem 3.2. Let $u$ denote the solution to (1.1) subject to $u(0) \equiv 1$. Suppose that $\sigma(0)=0$ and $\sigma(x) \neq 0$ for all $x>0$. Suppose that $f$ satisfies the reinforced Dalang's condition (1.6) for some $\alpha \in(0,1]$. Then, there is a version of the Malliavin derivative of $u(t, x)$ that satisfies

$$
D_{s, z} u(t, x)>0 \quad \text { for all } t>s \text { and } x \in \mathbb{R}^{d} \quad \text { a.s. }
$$

for all $s>0$ and $z \in \mathbb{R}^{d}$.

Proof. Choose and fix an arbitrary $s \geq 0$ and $z \in \mathbb{R}^{d}$. Proposition 3.2 of Chen and Huang [6] implies that, for all $(s, z)$ fixed, the random field $\left\{D_{s, z} u(t, x)\right\}_{(t, x) \in(s, \infty) \times \mathbb{R}^{d}}$ satisfies the following stochastic integral equation:

$$
D_{s, z} u(t, x)=\boldsymbol{p}_{t-s}(x-z) \sigma(u(s, z))+\int_{(s, t) \times \mathbb{R}^{d}} \boldsymbol{p}_{t-r}(x-y) \sigma^{\prime}(u(r, y)) D_{s, z} u(r, y) \eta(\mathrm{d} r \mathrm{~d} y) .
$$

Because $u(s, z)>0$, by the assumptions on $\sigma$, we see that $C_{s, z}:=\sigma(u(s, z))$ belongs to $\mathcal{F}_{s}$ and is strictly positive a.s. For $(s, z)$ fixed, the field $(t, x) \mapsto V_{s, z}(t, x):=C_{s, z}^{-1} D_{s, z} u(t, x)$ satisfies

$$
V_{s, z}(t, x)=p_{t-s}(x-z)+\int_{(s, t) \times \mathbb{R}^{d}} \boldsymbol{p}_{t-r}(x-y) \sigma^{\prime}(u(r, y)) V_{s, z}(r, y) \eta(\mathrm{d} r \mathrm{~d} y),
$$

where $(t, x) \in(s, \infty) \times \mathbb{R}^{d}$. Note that the above integral equation is nothing but the mild solution to the following variant of the stochastic heat equation:

$$
\left\{\begin{array}{l}
\frac{\partial}{\partial t} V_{s, z}(t, x)=\frac{1}{2} \Delta V_{s, z}(t, x)+H_{s, z}(t, x, \omega) V_{s, z}(t, x) \eta(t, x) \quad\left[t>s, x \in \mathbb{R}^{d}\right] \\
V_{s, z}(s, x)=\delta_{0}
\end{array}\right.
$$

where $H_{s, z}(t, x, \omega):=\sigma^{\prime}(u(t, x)(\omega))$. Notice that $\left|H_{s, z}(t, x, \omega)\right| \leq \operatorname{Lip}(\sigma)$ uniformly in $(s, z, t, x, \omega)$; see Remark 4.1. This is exactly the same setup as Theorem 1.8 of Chen and Huang [6] or Theorem 
1.5 of Chen et al [4] for the case when $d=1$ and $f=\delta_{0}$ (see also Theorem 1.5 of Chen and Huang [5]). Therefore, one can apply these references to conclude that

$$
\mathrm{P}\left\{V_{s, z}(t, x)>0, t>s, x \in \mathbb{R}^{d} \mid C_{s, z}>0\right\}=1,
$$

or in other words,

$$
\mathrm{P}\left\{D_{s, z} u(t, x)>0, t>s, x \in \mathbb{R}^{d}\right\}=\mathrm{P}\left\{V_{s, z}(t, x)>0, t>s, x \in \mathbb{R}^{d} \mid C_{s, z}>0\right\} \mathrm{P}\left\{C_{s, z}>0\right\}=1,
$$

for all $s \geq 0$ and , $z \in \mathbb{R}^{d}$, which is nothing but (3.8). This completes the proof of the theorem.

In order to derive the moment estimates of the Malliavin derivatives of the solution to (1.1), let us introduce some notation. Denote

$$
\Upsilon(\lambda):=\frac{2}{(2 \pi)^{d}} \int_{\mathbb{R}^{d}} \frac{\hat{f}(\mathrm{~d} z)}{2 \lambda+\|z\|^{2}} \quad \text { for all } \lambda>0 .
$$

Dalang's condition (1.5) ensures that $\Upsilon(\lambda)<\infty$ for all $\lambda>0$. In that case, $\Upsilon$ decreases strictly as $\lambda$ increases. Therefore, $\Upsilon$ has an inverse which we denote by

$$
\Lambda=\Upsilon^{-1} \text {. }
$$

The following variant of Chen et al [7, Theorem 6.4] shows a way in which $\Lambda$ can be used to control the size of the moments of the Malliavin derivative of the solution.

Lemma 3.3. For all real numbers $\varepsilon \in(0,1), t>0$, and $k \geq 2$, and for every $x \in \mathbb{R}^{d}$,

$$
\left\|D_{s, z} u(t, x)\right\|_{k} \leq C_{t, k, \varepsilon, \sigma} \boldsymbol{p}_{t-s}(x-z),
$$

valid for a.e. $(s, z) \in(0, t) \times \mathbb{R}^{d}$, where

$$
C_{t, k, \varepsilon, \sigma}:=\frac{8(|\sigma(0)| \vee \operatorname{Lip}(\sigma)) \mathrm{e}^{2 t \Lambda(\mathrm{a}(\varepsilon) / k)}}{\varepsilon^{3 / 2}} \quad \text { and } \quad \mathrm{a}(\varepsilon):=\frac{(1-\varepsilon)^{2}}{2^{(d+6) / 2}[|\sigma(0)| \vee \operatorname{Lip}(\sigma)]^{2}},
$$

where $1 \div 0:=\infty$

Proof. Choose and fix some $T>0$. We proved in Chen et al [7, Theorem 6.4] (see also [8, Lemma 4.2]) that, for each $t \in(0, T]$ and $x \in \mathbb{R}^{d}$, the random variable $u(t, x)$ is in the Gaussian Sobolev space $\mathbb{D}^{1, k}$ (see Nualart [32, Section 1.5$]$ ) for every $k \geq 2$, and that

$$
\left\|D_{s, z} u(t, x)\right\|_{k} \leq C_{T, k, \varepsilon, \sigma} \boldsymbol{p}_{t-s}(x-z),
$$

for a.e. $s \in(0, t)$ and $x, z \in \mathbb{R}^{d}$. Set $t=T$ and relabel $[T \leftrightarrow t]$ to derive the result.

Lemma 3.3 has nontrivial content if and only if $|\sigma(0)| \vee \operatorname{Lip}(\sigma)>0$. This is precisely when $\sigma \not \equiv 0$, which is equivalent to the statement that the stochastic PDE (1.1) is not identically the same as the nonrandom heat equation $\partial_{t} u=\frac{1}{2} \Delta u$.

In the particular case of the parabolic Anderson model, that is, when $\sigma(z)=z$ for all $z \in \mathbb{R}$, we are also able to estimate the moments of second-order Malliavin derivatives. Notice that when $\sigma(z)=z$ for all $z \in \mathbb{R}$, the constant defined in (3.10) has the form

$$
C_{t, k, \varepsilon}:=8 \varepsilon^{-3 / 2} \exp \left\{2 t \Lambda\left(\frac{(1-\varepsilon)^{2}}{2^{(d+6) / 2} k}\right)\right\} .
$$

Later on, for the proof of Theorem 2.5, we will need the following moment bound of second-order derivatives. 
Proposition 3.4. Suppose that $\sigma(z)=z$ for all $z \in \mathbb{R}$. Then, for all real numbers $\varepsilon \in(0,1)$, $t>0$, and $k \geq 2$, and for every $x \in \mathbb{R}^{d}$,

$$
\left\|D_{r, z} D_{s, y} u(t, x)\right\|_{k} \leq C_{t, k, \varepsilon}^{*}\left[\boldsymbol{p}_{t-s}(x-y) \boldsymbol{p}_{s-r}(y-z) \mathbf{1}_{(r, t)}(s)+\boldsymbol{p}_{t-r}(x-z) \boldsymbol{p}_{r-s}(z-y) \mathbf{1}_{(0, r)}(s)\right],
$$

valid for a.e. $(s, y),(r, z) \in(0, t) \times \mathbb{R}^{d}$, where

$$
C_{t, k, \varepsilon}^{*}=16 \varepsilon^{-2} \exp \left\{3 t \Lambda\left(\frac{(1-\varepsilon)^{2}}{2^{(d+6) / 2} k}\right)\right\} .
$$

Proof. The proof is fairly involved, though it mostly follows arguments that have been used earlier in simpler forms. Specifically, see the proof of Theorem 6.4 in [7]; see also Lemma 2.2 of [5]).

Consider the Picard iterations defined by $u_{0}(t, x):=1$ and

$$
u_{n+1}(t, x):=1+\int_{(0, t) \times \mathbb{R}^{d}} \boldsymbol{p}_{t-\tau}(x-y) u_{n}(\tau, \xi) \eta(\mathrm{d} \tau \mathrm{d} \xi)
$$

for all $n \in \mathbb{Z}_{+}, t \geq 0$, and $x \in \mathbb{R}^{d}$. The elementary properties of the Malliavin derivative and induction together show that $u_{n}(t, x) \in \cap_{k \geq 2} \mathbb{D}^{2, k}$ for every $(t, x) \in \mathbb{R}_{+} \times \mathbb{R}^{d}$ and $n \in \mathbb{Z}_{+}$. Moreover,

$$
D_{s, y} u_{n+1}(t, x)=\boldsymbol{p}_{t-s}(x-y) u_{n}(s, y)+\int_{(s, t) \times \mathbb{R}^{d}} \boldsymbol{p}_{t-\tau}(x-\xi) D_{s, y} u_{n}(\tau, \xi) \eta(\mathrm{d} \tau \mathrm{d} \xi),
$$

for almost every $(s, y) \in(0, t) \times \mathbb{R}^{d}$. Since $D_{s, y} u_{0}(t, x)=0$ a.s. for every $s, t \geq 0$ and $x, y \in \mathbb{R}^{d}$, we can, and in fact will, change the value of $D_{s, y} u_{n+1}(t, x)$ on a Lebesgue-null set of values of $(s, y)$ and define $D_{s, y} u_{n+1}(t, x)$ for every $(s, y) \in(0, t) \times \mathbb{R}^{d}$.

A second round of differentiation yields the following, after one more round of modifications on null sets:

$$
\begin{aligned}
D_{r, z} D_{s, y} u_{n+1}(t, x)=\boldsymbol{p}_{t-s}(x-y) D_{r, z} u_{n}(s, y) & +\boldsymbol{p}_{t-r}(x-z) D_{s, y} u_{n}(r, z) \\
& +\int_{(s \vee r, t) \times \mathbb{R}^{d}} \boldsymbol{p}_{t-\tau}(x-\xi) D_{r, z} D_{s, y} u_{n}(\tau, \xi) \eta(\mathrm{d} \tau \mathrm{d} \xi),
\end{aligned}
$$

for every $(s, y),(r, z) \in(0, t) \times \mathbb{R}^{d}$.

According to the proof of Theorem 6.4 in Chen et al [7], and thanks to the same argument that was used in Lemma 3.9, the estimate (3.9) holds also - with the same constant $C_{t, k, \varepsilon, \sigma}$ - when we replace $D_{s, z} u(t, x)$ by $D_{s, z} u_{n+1}(t, x)$. Indeed, Chen et al (ibid.) first bound the moments of $D_{s, z} u_{n}(t, x)$ and then obtain (3.9) by passing to the limit, as $n \rightarrow \infty$. In this way, we find that

$$
\begin{array}{r}
\left\|D_{r, z} D_{s, y} u_{n+1}(t, x)\right\|_{k} \leq C_{t, k, \varepsilon}\left[\boldsymbol{p}_{t-s}(x-y)\right. \\
\left.+\| \boldsymbol{p}_{s-r}(y-z) \mathbf{1}_{(r, t)}(s)+\boldsymbol{p}_{t-r}(x-z) \boldsymbol{p}_{r-s}(z-y) \mathbf{1}_{(0, r)}(s)\right] \\
\boldsymbol{p}_{t-\tau}(x-\xi) D_{r, z} D_{s, y} u_{n}(\tau, \xi) \eta(\mathrm{d} \tau \mathrm{d} \xi) \|_{k},
\end{array}
$$

for the same constant $C_{t, k, \varepsilon}$ that was defined earlier in (3.11). We can now apply the BurkholderDavis-Gundy inequality [2], using with the Carlen and Kreé [3] bounds on the optimal constants, in order to find that

$$
\begin{aligned}
& \mathrm{E}\left(\left|\int_{(s \vee r, t) \times \mathbb{R}^{d}} \boldsymbol{p}_{t-\tau}(x-\xi) D_{r, z} D_{s, y} u_{n+1}(\tau, \xi) \eta(\mathrm{d} \tau \mathrm{d} \xi)\right|^{k}\right) \\
& \leq(2 \sqrt{k})^{k} \mathrm{E}\left(\left|\int_{r \vee s}^{t} \mathrm{~d} \tau \int_{\mathbb{R}^{d}} \mathrm{~d} \xi \int_{\mathbb{R}^{d}} f\left(\mathrm{~d} \xi^{\prime}\right) \boldsymbol{p}_{t-\tau}\left(x-\xi+\xi^{\prime}\right) \boldsymbol{p}_{t-\tau}(x-\xi) \mathcal{T}_{n}\left(\tau, \xi-\xi^{\prime}\right) \mathcal{T}_{n}\left(\tau, \xi^{\prime}\right)\right|^{k / 2}\right),
\end{aligned}
$$


where

$$
\mathcal{T}_{n}(\tau, a):=D_{r, z} D_{s, y} u_{n}(\tau, a) \quad \text { for every } \tau>0 \text { and } a \in \mathbb{R}^{d} .
$$

We combine the above with Minkowski's inequality and Hölder's inequality, and deduce that

$$
\begin{aligned}
& \left\|\int_{(s \vee r, t) \times \mathbb{R}^{d}} \boldsymbol{p}_{t-\tau}(x-\xi) D_{r, z} D_{s, y} u_{n+1}(\tau, \xi) \eta(\mathrm{d} \tau \mathrm{d} \xi)\right\|_{k} \\
& \leq 2 \sqrt{k}\left[\int_{r \vee s}^{t} \mathrm{~d} \tau \int_{\mathbb{R}^{d}} \mathrm{~d} \xi \int_{\mathbb{R}^{d}} f\left(\mathrm{~d} \xi^{\prime}\right) \boldsymbol{p}_{t-\tau}\left(x-\xi+\xi^{\prime}\right) \boldsymbol{p}_{t-\tau}(x-\xi)\left\|\mathcal{T}_{n}\left(\tau, \xi-\xi^{\prime}\right)\right\|_{k}\left\|\mathcal{T}_{n}\left(\tau, \xi^{\prime}\right)\right\|_{k}\right]^{1 / 2} .
\end{aligned}
$$

The preceding computations yield the following inequality on the $L^{k}(\Omega)$-norm of the second Malliavin derivative of $u_{n+1}(t, x)$ :

$$
\begin{gathered}
\left\|D_{r, z} D_{s, y} u_{n+1}(t, x)\right\|_{k} \leq C_{t, k, \varepsilon}\left[\mathcal{P}_{s, r, t}(y, z ; x) \mathbf{1}_{(r, t)}(s)+\mathcal{P}_{r, s, t}(z, y ; x) \mathbf{1}_{(0, r)}(s)\right] \\
+2 \sqrt{k}\left[\int_{r \vee s}^{t} \mathrm{~d} \tau \int_{\mathbb{R}^{d}} \mathrm{~d} \xi \int_{\mathbb{R}^{d}} f\left(\mathrm{~d} \xi^{\prime}\right) \boldsymbol{p}_{t-\tau}\left(x-\xi+\xi^{\prime}\right) \boldsymbol{p}_{t-\tau}(x-\xi)\left\|\mathcal{T}_{n}\left(\tau, \xi-\xi^{\prime}\right)\right\|_{k}\left\|\mathcal{T}_{n}\left(\tau, \xi^{\prime}\right)\right\|_{k}\right]^{1 / 2},
\end{gathered}
$$

where

$$
\mathcal{P}_{s, r, t}(y, z ; x)=\boldsymbol{p}_{t-s}(x-y) \boldsymbol{p}_{s-r}(y-z) .
$$

Set $\gamma:=2^{d / 2} 16 k$ and $h_{0}(t):=1$ for all $t>0$, and define

$$
h_{n}(t):=\int_{0}^{t} h_{n-1}(s)\left(\boldsymbol{p}_{2(t-s)} * f\right)(0) \mathrm{d} s \quad \text { for all } t>0 \text { and } n \in \mathbb{N} .
$$

We claim that, for every $n \in \mathbb{Z}_{+}$,

$$
\begin{aligned}
& \left\|D_{r, z} D_{s, y} u_{n}(t, x)\right\|_{k} \\
& \leq \sqrt{2} C_{t, k, \varepsilon}\left[\mathcal{P}_{s, r, t}(y, z ; x) \sqrt{\sum_{i=0}^{n} \gamma^{i} h_{i}(t-s)} \mathbf{1}_{(r, t)}(s)+\mathcal{P}_{r, s, t}(z, y ; x) \sqrt{\sum_{i=0}^{n} \gamma^{i} h_{i}(t-r) \mathbf{1}_{(0, r)}(s)}\right] .
\end{aligned}
$$

We prove (3.13) using induction on $n$.

Eq. (3.13) holds for $n=0$ since $u_{0} \equiv 1$ whence $D_{r, z} D_{s, y} u_{0}(t, x)=0$ a.s. We now suppose that (3.13) with $n$ replaced by any arbitrary $m=0, \ldots, n-1$ and seek to verify that (3.13) holds for $n \geq 1$.

Indeed, by the induction hypothesis,

$$
\begin{gathered}
\int_{r \vee s}^{t} \mathrm{~d} \tau \int_{\mathbb{R}^{d}} \mathrm{~d} \xi \int_{\mathbb{R}^{d}} f\left(\mathrm{~d} \xi^{\prime}\right) \boldsymbol{p}_{t-\tau}\left(x-\xi+\xi^{\prime}\right) \boldsymbol{p}_{t-\tau}(x-\xi)\left\|\mathcal{T}_{n-1}\left(\tau, \xi-\xi^{\prime}\right)\right\|_{k}\left\|\mathcal{T}_{n-1}(\tau, \xi)\right\|_{k} \\
\leq 2 \mathbf{1}_{(r, t)}(s) C_{t, k, \varepsilon}^{2} \sum_{i=0}^{n-1} \gamma^{i} \int_{s}^{t} \mathrm{~d} \tau \int_{\mathbb{R}^{d}} \mathrm{~d} \xi \int_{\mathbb{R}^{d}} f\left(\mathrm{~d} \xi^{\prime}\right) \\
\boldsymbol{p}_{t-\tau}\left(x-\xi+\xi^{\prime}\right) \boldsymbol{p}_{t-\tau}(x-\xi) \\
\quad \times \mathcal{P}_{s, r, \tau}\left(y, z ; \xi-\xi^{\prime}\right) \mathcal{P}_{s, r, \tau}(y, z ; \xi) h_{i}(\tau-s) \\
+2 \mathbf{1}_{(0, r)}(s) C_{t, k, \varepsilon}^{2} \sum_{i=0}^{n-1} \gamma^{i} \int_{r}^{t} \mathrm{~d} \tau \int_{\mathbb{R}^{d}} \mathrm{~d} \xi \int_{\mathbb{R}^{d}} f\left(\mathrm{~d} \xi^{\prime}\right) \boldsymbol{p}_{t-\tau}\left(x-\xi+\xi^{\prime}\right) \boldsymbol{p}_{t-\tau}(x-\xi) \\
\quad \times \mathcal{P}_{r, s, \tau}\left(z, y ; \xi-\xi^{\prime}\right) \mathcal{P}_{r, s, \tau}(z, y ; \xi) h_{i}(\tau-r) \\
=: 2 \mathbf{1}_{(r, t)}(s) \Phi(s, r ; y, z)+2 \mathbf{1}_{(0, r)}(s) \Phi(r, s ; z, y) .
\end{gathered}
$$


Because

$$
\boldsymbol{p}_{t-s}(x-y) \boldsymbol{p}_{s}(y-z)=\boldsymbol{p}_{t}(x-z) \boldsymbol{p}_{s(t-s) / t}\left(y-z-\frac{s}{t}(x-z)\right)
$$

it follows that

$$
\begin{aligned}
\Phi(s, r ; y, z)= & C_{t, k, \varepsilon}^{2} \boldsymbol{p}_{s-r}^{2}(y-z) \sum_{i=0}^{n-1} \gamma^{i} \int_{s}^{t} \mathrm{~d} \tau \int_{\mathbb{R}^{d}} \mathrm{~d} \xi \int_{\mathbb{R}^{d}} f\left(\mathrm{~d} \xi^{\prime}\right) \boldsymbol{p}_{t-\tau}\left(x-\xi+\xi^{\prime}\right) \boldsymbol{p}_{t-\tau}(x-\xi) \\
& \times \boldsymbol{p}_{\tau-s}\left(\xi-\xi^{\prime}-y\right) \boldsymbol{p}_{\tau-s}(\xi-y) h_{i}(\tau-s) \\
= & C_{t, k, \varepsilon}^{2} \boldsymbol{p}_{s-r}^{2}(y-z) \boldsymbol{p}_{t-s}^{2}(x-y) \\
& \times \sum_{i=0}^{n-1} \gamma^{i} \int_{s}^{t} \mathrm{~d} \tau \int_{\mathbb{R}^{d}} \mathrm{~d} \xi \int_{\mathbb{R}^{d}} f\left(\mathrm{~d} \xi^{\prime}\right) \boldsymbol{p}_{(t-\tau)(\tau-s) /(t-s)}\left(\xi-\xi^{\prime}-y-\frac{\tau-s}{t-s}(x-y)\right) \\
& \times \boldsymbol{p}_{(t-\tau)(\tau-s) /(t-s)}\left(\xi-y-\frac{\tau-s}{t-s}(x-y)\right) h_{i}(\tau-s) .
\end{aligned}
$$

Therefore, the semigroup property of the heat kernel, together with its symmetry, yield the following:

$$
\Phi(s, r ; y, z)=C_{t, k, \varepsilon}^{2} \boldsymbol{p}_{s-r}^{2}(y-z) \boldsymbol{p}_{t-s}^{2}(x-y) \sum_{i=0}^{n-1} \gamma^{i} \int_{s}^{t} \mathrm{~d} \tau \int_{\mathbb{R}^{d}} f\left(\mathrm{~d} \xi^{\prime}\right) \boldsymbol{p}_{2(t-\tau)(\tau-s) /(t-s)}\left(\xi^{\prime}\right) h_{i}(\tau-s) .
$$

According to Lemma 6.6 of Chen et al [7],

$$
\int_{s}^{t} g(\tau-s) p_{2(\tau-s)(t-\tau) /(t-s)}(y) \mathrm{d} \tau \leq 2^{(d+2) / 2} \int_{s}^{t} g(\tau-s) p_{2(t-\tau)}(y) \mathrm{d} \tau
$$

for every nondecreasing function $g:(0, t-s) \rightarrow \mathbb{R}$. In accord with Lemma 2.6 of Chen and Kim [10], every $h_{i}$ is nondecreasing (this fact follows from induction on $i$ ). Therefore, it follows from the above [with $g \leftrightarrow h_{i}$ ] and the definition of the $h_{i}$ 's that

$$
\begin{aligned}
\Phi(s, r ; y, z) & \leq C_{t, k, \varepsilon}^{2} 2^{(d+2) / 2} \boldsymbol{p}_{s-r}^{2}(y-z) \boldsymbol{p}_{t-s}^{2}(x-y) \sum_{i=0}^{n-1} \gamma^{i} h_{i+1}(t-s) \\
& =\frac{C_{t, k, \varepsilon}^{2}}{8 k} \boldsymbol{p}_{s-r}^{2}(y-z) \boldsymbol{p}_{t-s}^{2}(x-y) \sum_{i=1}^{n} \gamma^{i} h_{i}(t-s),
\end{aligned}
$$

the last line holding since we originally made the special choice, $\gamma=2^{d / 2} 16 k$. Consequently,

$$
\begin{aligned}
& \left\|D_{r, z} D_{s, y} u_{n}(t, x)\right\|_{k} \\
& \leq C_{t, k, \varepsilon}\left[\mathcal{P}_{s, r, t}(y, z ; x) \mathbf{1}_{(r, t)}(s)+\mathcal{P}_{r, s, t}(z, y ; x) \mathbf{1}_{(0, r)}(s)\right] \\
& +\sqrt{2} C_{t, k, \varepsilon}\left[\begin{array}{l}
\mathbf{1}_{(r, t)}(s) \boldsymbol{p}_{s-r}(y-z) \boldsymbol{p}_{t-s}(x-y)\left(\sum_{i=1}^{n} \gamma^{i} h_{i}(t-s)\right)^{1 / 2} \\
\left.\quad+\mathbf{1}_{(0, r)}(s) \boldsymbol{p}_{r-s}(z-y) \boldsymbol{p}_{t-r}(x-y)\left(\sum_{i=1}^{n} \gamma^{i} h_{i}(t-r)\right)^{1 / 2}\right],
\end{array}\right.
\end{aligned}
$$

which proves (3.13). 
We appeal to the nondecreasing property of the $h_{n}$ 's once again in order to deduce from (3.13) that

$$
\left\|D_{r, z} D_{s, y} u_{n+1}(t, x)\right\|_{k} \leq \sqrt{2} C_{t, k, \varepsilon} \boldsymbol{P}(s, r, t ; y, z, x) \sqrt{\sum_{i=1}^{n} \gamma^{i} h_{i}(t)},
$$

where

$$
\boldsymbol{P}(s, r, t ; y, z, x):=\mathbf{1}_{(r, t)}(s) \boldsymbol{p}_{s-r}(y-z) \boldsymbol{p}_{t-s}(x-y)+\mathbf{1}_{(0, r)}(s) \boldsymbol{p}_{r-s}(z-y) \boldsymbol{p}_{t-r}(x-y) .
$$

We now let $n \rightarrow \infty$ in order to obtain

$$
\left\|D_{r, z} D_{s, y} u(t, x)\right\|_{k} \leq \sqrt{2} C_{t, k, \varepsilon} \boldsymbol{P}(s, r, t ; y, z, x) \sqrt{\sum_{i=1}^{\infty} \gamma^{i} h_{i}(t)} .
$$

The details are the same as its counterpart in the proof of Theorem 6.4 of [7]. Therefore, we skip those details. Because the Plancherel theorem implies that $\int_{0}^{\infty} \exp (-\lambda t)\left(\boldsymbol{p}_{t} * f\right)(0) \mathrm{d} t=\Upsilon(\lambda)$, Lemma 6.7 of Chen et al [7] implies that

$$
\sum_{i=1}^{\infty} \gamma^{i} h_{i}(t) \leq \frac{\exp (2 \lambda t)}{1-\frac{1}{2} \gamma \Upsilon(\lambda)}
$$

for all large enough $\lambda>0$ that satisfy $2>\gamma \Upsilon(\lambda)$. We now follow the proof of Lemma 4.2 in [8] and choose the particular value,

$$
\lambda=\Lambda\left(\frac{(1-\varepsilon)^{2}}{k 2^{(d+6) / 2}}\right) .
$$

This choice yields

$$
\left\|D_{r, z} D_{s, y} u(t, x)\right\|_{k} \leq 2 \varepsilon^{-1 / 2} C_{t, k, \varepsilon} \exp \left\{t \Lambda\left(\frac{(1-\varepsilon)^{2}}{k 2^{(d+6) / 2}}\right)\right\} \boldsymbol{P}(s, r, t ; y, z, x),
$$

and completes the proof of Proposition 3.4.

\subsection{Malliavin derivatives and shifts of the noise}

In the general setup of Malliavin calculus, the Hilbert space $L^{2}(\Omega)$ includes square-integrable random variables that are functions of the isonormal process $h \mapsto \eta(h)$; see (3.1). Thus, we identify every random variable $F \in L^{2}(\Omega)$ with a function $F(\eta)$ of the underlying noise $\eta$. Note that this function need not be local. That is, the instance $F(\omega)$ of the random variable $F[\omega \in \Omega]$ need not be a function of the instance $\eta(\omega)$ of the noise. To see how this can happen, choose and fix some $h \in \mathcal{H}$ and consider the random variable $F_{0}=\eta(h)$. Because Wiener integrals cannot be defined pathwise (unless $h$ is sufficiently smooth), this shows that the random variable $F_{0}$ is not a local function of $\eta$, though it is of course a function of $\eta$.

Let us follow the proof of Lemma 7.1 in [7] and define, for every $y \in \mathbb{R}^{d}$, the shifted Gaussian noise $\eta_{y}$, and the corresponding Gaussian family of random variables in the following manner: For all $\varphi \in \mathcal{H}$ and $y \in \mathbb{R}^{d}$ we set

$$
\eta_{y}(\varphi):=\eta\left(\varphi_{y}\right)
$$

where $\varphi_{y}(s, x):=\varphi(s, x-y)$. We remark that the elements of the Hilbert space $\mathcal{H}_{0}$ are generalized functions, so the shifted element $\varphi_{y}$ should be defined as in distribution theory: $\left(\varphi_{y}, \psi\right):=\left(\varphi, \psi_{-y}\right)$ 
for every smooth function $\psi$ of rapid decrease. By the Wiener isometry for Wiener stochastic integrals, $\eta_{y}$ has the same law as $\eta$ for every $y \in \mathbb{R}^{d}$. As in Chen et al [7, Lemma 7.1], we define a family of shift operators $\left\{\theta_{y}\right\}_{y \in \mathbb{R}^{d}}$ that act on every random variable $F \in L^{2}(\Omega)$ to create new random variables $\left\{F \circ \theta_{y}\right\}_{y \in \mathbb{R}^{d}}$ in $L^{2}(\Omega)$ as follows:

$$
\left(F \circ \theta_{y}\right)(\eta):=F\left(\eta_{y}\right) \quad \text { for every } y \in \mathbb{R}^{d} .
$$

Lemma 3.5. Choose and fix some $y \in \mathbb{R}^{d}$ and $F \in \mathbb{D}^{1,2}$. Then, a.s., $D\left(F \circ \theta_{y}\right)=(D F)_{y} \circ \theta_{y}$.

Proof. We first examine the case that $F(\eta)=\eta(\varphi)$ for some $\varphi \in \mathcal{H}$. On one hand, $(D F)_{y}=\varphi_{y}$. Since $\varphi$ is non random, this proves that $(D F)_{y} \circ \theta_{a}=\varphi_{y}$ a.s. for all $a \in \mathbb{R}^{d}$. This holds in particular when $a=y$. On the other hand, $F \circ \theta_{y}=\eta_{y}(\varphi)=\eta\left(\varphi_{y}\right)$ and hence $D\left(F \circ \theta_{y}\right)=\varphi_{y}$. This proves the lemma in the case that $F=\eta(\varphi)$.

The preceding special case and the chain rule of Malliavin calculus (see Nualart [32, Proposition 1.2.3]) together imply that the lemma holds also when $F(\eta)=\prod_{j=1}^{k} \Phi_{j}\left(\eta\left(\varphi_{j}\right)\right)$ when $\Phi_{1}, \ldots, \Phi_{k}$ : $\mathbb{R} \rightarrow \mathbb{R}$ are smooth and grow at most polynomially, and $\varphi_{1}, \ldots, \varphi_{k} \in \mathcal{H}$. The general result follows from this case and density; see Nualart [32, Proposition 1.1.1].

\subsection{An abstract ergodicity result}

In [7] we proved that the infinite dimensional random variable $u(t)$ is [spatially] ergodic for every $t>0$. The following is a non-trivial variation on that and uses similar ideas.

Throughout this section, we suspend the finiteness portion of assumption (1.11).

Because of the above, the Fourier transform $\hat{f}$ of $f$ is a positive-definite tempered measure that need not be bounded.

Theorem 3.6. To every random variable $G \in L^{2}(\Omega)$ we associate a random field - also denoted by $G-$ via $G(x):=G \circ \theta_{x}$ for all $x \in \mathbb{R}^{d}$. Then, $\{G(x)\}_{x \in \mathbb{R}^{d}}$ is stationary. Moreover, the following are equivalent:

1. $\left\{G(x\}_{x \in \mathbb{R}^{d}}\right.$ is ergodic for every $G \in L^{2}(\Omega)$ such that $x \mapsto G(x)$ is continuous in probability;

2. $\hat{f}\{0\}=0$.

The spectral condition $2, \hat{f}\{0\}=0$, is equivalent to either one of the following:

1. $\hat{f}$ has no atoms;

2. $f\left\{x \in \mathbb{R}^{d}:\|x\|<r\right\}=o\left(r^{d}\right)$ as $r \rightarrow \infty$.

See Chen et al [7] for details.

Before we prove Theorem 3.6, we point out a further generalization which is noteworthy (though we will not need it here).

Remark 3.7. One can make only small adjustments to the proof of Theorem 3.6 in order to see that if $\boldsymbol{G}=\left(G_{1}, \ldots, G_{m}\right)$ is an $m$-dimensional random vector with $G_{i} \in L^{2}(\Omega)$ for every $i=1, \ldots, m$, then $\left\{\boldsymbol{G} \circ \theta_{x}\right\}_{x \in \mathbb{R}^{d}}$ is stationary and ergodic, where $\left(\boldsymbol{G} \circ \theta_{x}\right)(\eta):=\boldsymbol{G}\left(\eta_{x}\right)$, the same as in the case $m=1$. This particular phrasing of Theorem 3.6 extends the ergodicity result of [7] since it was shown in the latter reference that $u(t, x+y)=u(t, x) \circ \theta_{y}$ a.s. for all $t \geq 0$ and $x, y \in \mathbb{R}^{d}$. In fact, this phrasing can be viewed to be an infinite-dimensional extension of a classical result of Maruyama [27]; see also Dym and McKean [17]. 
Proof of Theorem 3.6. Stationarity is immediate; we prove only ergodicity.

First, suppose that $\{G(x)\}_{x \in \mathbb{R}^{d}}$ is ergodic for every $G \in L^{2}(\Omega)$ such that $x \mapsto G(x)$ is continuous in probability. ${ }^{4}$ Consider random variables of the form $G=\eta\left(\mathbf{1}_{[0,1]} \otimes \varphi\right)$ where $\varphi \in \mathscr{S}\left(\mathbb{R}^{d}\right)$ is a probability density function. In this case, $G(x)=\int_{(0,1) \times \mathbb{R}^{d}} \varphi(y-x) \eta(\mathrm{d} s \mathrm{~d} y)$ is continuous in $L^{2}(\Omega)$ and hence in probability. Indeed,

$$
\operatorname{Cov}[G(x), G(0)]=\int_{\mathbb{R}^{d}} \varphi(y-x)(\varphi * f)(y) \mathrm{d} y \quad \text { for all } x \in \mathbb{R}^{d} .
$$

Consequently, $\left\|G(x)-G\left(x^{\prime}\right)\right\|_{2}^{2}=2 \int_{\mathbb{R}^{d}}\left[\varphi(y)-\varphi\left(y-x+x^{\prime}\right)\right](\varphi * f)(y) \mathrm{d} y \rightarrow 0$ as $x^{\prime} \rightarrow x$. Also, the assumed ergodicity implies among other things that

$$
\left\|N^{-d} \int_{[0, N] d} G(x) \mathrm{d} x\right\|_{2}^{2}=\left(I_{N} * \tilde{I}_{N} * \tilde{\varphi} * \varphi * f\right)(0) \rightarrow 0 \quad \text { as } N \rightarrow \infty,
$$

where $I_{N}:=N^{-d} \mathbf{1}_{[0, N]^{d}}$ and $\tilde{Q}(z):=Q(-z)$ for all $z \in \mathbb{R}^{d}$ and all $Q: \mathbb{R}^{d} \rightarrow \mathbb{R}$. Because

$$
\left(I_{N} * \tilde{I}_{N} * \tilde{\varphi} * \varphi * f\right)(0)=\frac{1}{(2 \pi)^{d}} \int_{\mathbb{R}^{d}}|\hat{\varphi}(z)|^{2} \prod_{j=1}^{d}\left(\frac{1-\cos \left(N z_{j}\right)}{\left(N z_{j}\right)^{2}}\right) \hat{f}(\mathrm{~d} z),
$$

and $\hat{\varphi}(0)=1$, the dominated convergence theorem implies that $\hat{f}\{0\}=0$.

For the more interesting half of the proof we assume $\hat{f}\{0\}=0$, and extend the ideas of the proof of Lemma 7.2 of Chen et al [7].

Define $\mathcal{G}(x):=\prod_{j=1}^{k} g_{j}\left(G\left(x+\zeta^{j}\right)\right)$, where $k \geq 1, \zeta^{1}, \ldots, \zeta^{k} \in \mathbb{R}^{d}$, and $g_{1}, \ldots, g_{k}: \mathbb{R} \rightarrow \mathbb{R}$ are Lipschitz-continuous and bounded functions that satisfy $g_{j}(0)=0$ and $\operatorname{Lip}\left(g_{j}\right)=1$ for every $j=1, \ldots, k$. Since $\mathcal{G}$ is a stationary random field, the ergodic theorem reduces the problem to proving the following:

$$
V_{N}(G):=\operatorname{Var}\left(N^{-d} \int_{[0, N]^{d}} \mathcal{G}(x) \mathrm{d} x\right) \rightarrow 0 \quad \text { as } N \rightarrow \infty .
$$

See [7, Lemma 7.2 ] for the details of this argument.

The bulk of the proof is concerned with the proposition in the special case that the random variable $G$ has the form,

$$
G=h\left(\eta\left(\psi_{1}\right), \ldots, \eta\left(\psi_{m}\right)\right),
$$

where $h \in C_{c}^{\infty}\left(\mathbb{R}^{m}\right)$ and $\psi_{1}, \ldots, \psi_{m} \in C_{c}\left(\mathbb{R}_{+} \times \mathbb{R}^{d}\right)$. Let us first understand why it is enough to study $G$ 's of the form (3.15).

Given an arbitrary random variable $G \in L^{2}(\Omega)$ and a number $\varepsilon>0$ we can find a random variable $\bar{G}$ that can be written as the right-hand side of (3.15) and satisfies $\|G-\bar{G}\|_{2} \leq \varepsilon$; see Nualart [32, Proposition 1.1.1]. The argument that we used at the very beginning portion of the proof can be recycled to show that $x \mapsto \bar{G}(x):=\bar{G} \circ \theta_{x}$ is continuous in $L^{2}(\Omega)$ and hence in probability. Let $\overline{\mathcal{G}}$ denote the analogue of $\mathcal{G}$ but with $G$ replaced by $\bar{G}$ everywhere. By the triangle inequality,

$$
\begin{aligned}
\left|\sqrt{V_{N}(G)}-\sqrt{V_{N}(\bar{G})}\right| & \leq\left\|\left(N^{-d} \int_{[0, N]^{d}} \mathcal{G}(x) \mathrm{d} x-\mathrm{E} \mathcal{G}(0)\right)-\left(N^{-d} \int_{[0, N]^{d}} \overline{\mathcal{G}}(x) \mathrm{d} x-\mathrm{E} \overline{\mathcal{G}}(0)\right)\right\|_{2} \\
& \leq 2 \varepsilon .
\end{aligned}
$$

\footnotetext{
${ }^{4}$ Continuity in probability is here only to ensure that $x \mapsto G(x)$ has a Lebesgue-measurable version.
} 
If we could prove (3.14) for all random variables of the form (3.15), then $\lim _{N \rightarrow \infty} V_{N}(\bar{G})=0$ whence $\limsup _{N \rightarrow \infty} V_{N}(G) \leq 4 \varepsilon^{2}$ by the above. Since $\varepsilon>0$ is arbitrary this shows that it is enough to prove (3.14) for random variables of the form (3.15).

Suppose now that $G$ has the form (3.15). By the Poincaré inequality (3.4),

$$
V_{N}(G) \leq \mathrm{E}\left(\left\|N^{-d} \int_{[0, N]^{d}} D \mathcal{G}(x) \mathrm{d} x\right\|_{\mathcal{H}}^{2}\right)=N^{-2 d} \iint_{[0, N]^{2 d}} \mathrm{~d} x \mathrm{~d} y \operatorname{E}\left[\langle D \mathcal{G}(x), D \mathcal{G}(y)\rangle_{\mathcal{H}}\right] .
$$

Before we study the expectation inside the double integral, note using the chain rule of the Malliavin calculus (see Nualart [32, Proposition 1.2.3]) that

$$
D_{s, z} \mathcal{G}(x)=\sum_{j_{0}=1}^{k}\left(\prod_{j=1, j \neq j_{0}}^{k} g_{j}\left(G\left(x+\zeta^{j}\right)\right)\right) D_{s, z} G\left(x+\zeta^{j_{0}}\right),
$$

and observe that $D_{s, z} G\left(x+\zeta^{j_{0}}\right)=\sum_{i=1}^{m}\left(\partial_{i} h\right)(Y) \psi_{i}\left(s, z-x-\zeta^{j_{0}}\right)$, where $Y$ denotes the $m$ dimensional Gaussian random vector $\left(\eta_{x+\zeta^{j_{1}}}\left(\psi_{1}\right), \ldots, \eta_{x+\zeta^{j m}}\left(\psi_{m}\right)\right)$. Thus,

$$
\begin{aligned}
\left\|D_{s, z} \mathcal{G}(x)\right\|_{2} & \leq \operatorname{Lip}(h) \sum_{j_{0}=1}^{k}\left\|\prod_{j=1, j \neq j_{0}}^{k} g_{j}\left(G\left(x+\zeta^{j}\right)\right)\right\| \sum_{2}\left|\sum_{i=1}^{m}\right| \psi_{i}\left(s, z-x-\zeta^{j_{0}}\right) \mid \\
& \leq \operatorname{Lip}(h) \sup _{\substack{1 \leq j \leq k \\
w \in \mathbb{R}}}\left|g_{j}(w)\right|^{k-1} \sum_{\substack{1 \leq j_{j} \leq k \\
1 \leq i \leq m}}\left|\psi_{i}\left(s, z-x-\zeta^{j_{0}}\right)\right|:=L \sum_{\substack{1 \leq j_{0} \leq k \\
1 \leq i \leq m}}\left|\psi_{i}\left(s, z-x-\zeta^{j_{0}}\right)\right| .
\end{aligned}
$$

Consequently, the Walsh-isometry for stochastic integrals yields

$$
\begin{aligned}
& \mathrm{E}\left[\langle D \mathcal{G}(x), D \mathcal{G}(y)\rangle_{\mathcal{H}}\right]=\int_{0}^{t} \mathrm{~d} s \int_{\mathbb{R}^{d}} \mathrm{~d} a \int_{\mathbb{R}^{d}} f(\mathrm{~d} b) \mathrm{E}\left[D_{s, a} \mathcal{G}(x) D_{s, a-b} \mathcal{G}(y)\right] \\
& \leq L^{2} \sum_{\substack{1 \leq j_{0}, j_{1} \leq k \\
1 \leq i_{0}, i_{1} \leq m}} \int_{0}^{t} \mathrm{~d} s \int_{\mathbb{R}^{d}} \mathrm{~d} a \int_{\mathbb{R}^{d}} f(\mathrm{~d} b)\left|\psi_{i_{0}}\left(s, a-x-\zeta^{j_{0}}\right) \psi_{i_{1}}\left(s, a-b-y-\zeta^{j_{1}}\right)\right| .
\end{aligned}
$$

Recall that $I_{N}:=N^{-d} \mathbf{1}_{[0, N]}$, and integrate the preceding displayed expression $\left[I_{N}(x) \mathrm{d} x I_{N}(y) \mathrm{d} y\right]$ in order to deduce from Fubini's theorem that

$$
V_{N}(G) \leq L^{2} \sum_{\substack{1 \leq j_{0}, j_{1} \leq k \\ 1 \leq i_{0}, i_{1} \leq m}} \int_{0}^{t} \mathrm{~d} s\left(I_{N} * \tilde{I}_{N} * f *\left|\psi_{i_{0}}(s)\right| *\left|\tilde{\psi}_{i_{1}}(s)\right|\right)\left(\zeta^{j_{0}}-\zeta^{j_{1}}\right),
$$

where $Q(s)=Q(s, \bullet)$ for all $s \geq 0$ and space-time functions $Q: \mathbb{R}_{+} \times \mathbb{R}^{d} \rightarrow \mathbb{R}$, and $\tilde{q}(x)=q(-x)$ for all $x \in \mathbb{R}^{d}$ and $q: \mathbb{R}^{d} \rightarrow \mathbb{R}$. Since $I_{N} * \tilde{I}_{N} * f$ is a continuous, positive definite function, it is maximized at the origin. This is a consequence of the Bochner-Hergloz theorem of classical Fourier analysis, and found in introductory probability textbooks. This yields

$$
V_{N}(G) \leq(k L)^{2}\left(I_{N} * \tilde{I}_{N} * f\right)(0) \int_{0}^{t}\left(\sum_{i=1}^{m}\left\|\tilde{\psi}_{i}(s)\right\|_{L^{1}\left(\mathbb{R}^{d}\right)}\right)^{2} \mathrm{~d} s .
$$

According to Chen et al [7, Proof of Proposition 3.7], $\left(I_{N} * \tilde{I}_{N} * f\right)(0) \rightarrow 0$ as $N \rightarrow \infty$ because $\hat{f}\{0\}=0$. This establishes (3.14) when $G$ has the form (3.15), and completes the proof of the proposition. 


\section{CLT: Proof of Theorem 2.1}

Before we begin the proof properly, let us pause to make two elementary remarks, one from real analysis and the other from information theory.

Remark 4.1. If $g \in$ Lip then according to Rademacher's theorem, the weak derivative $g^{\prime}$ of $g$ is a.e.-defined and satisfies $\left|g^{\prime}\right| \leq \operatorname{Lip}(g)$ a.e.; see for example Federer [18, Theorem 3.1.6].

Remark 4.2. If $0<c_{2}<c_{1}$, then

$$
d_{\mathrm{TV}}\left[\mathrm{N}\left(0, c_{1}\right), \mathrm{N}\left(0, c_{2}\right)\right] \leq \frac{1}{2} \sqrt{\frac{c_{1}-c_{2}}{c_{2}}}
$$

One can prove this quickly using Pinsker's inequality (see, for example, Cover and Thomas [12]). Indeed, Pinsker's inequality tells us that $2\left[d_{\mathrm{TV}}\left(\sqrt{c_{1}} Z, \sqrt{c_{2}} Z\right)\right]^{2}$ is at most the relative entropy of $\mathrm{N}\left(0, c_{1}\right)$ with respect to $\mathrm{N}\left(0, c_{2}\right)$, which is $-\left|\log \left(c_{1} / c_{2}\right)\right|+\left(c_{1}-c_{2}\right) /\left(2 c_{2}\right) \leq\left(c_{1}-c_{2}\right) /\left(2 c_{2}\right)$.

According to Chen et al [8, Lemma 4.3] or (3.9), it is easy to see that in case of Lipschitz $g$,

$$
\mathcal{S}_{N, t}(g) \in \cap_{k \geq 2} \mathbb{D}^{1, k} \quad \text { for all } N>0,
$$

and

$$
D_{s, z} \mathcal{S}_{N, t}(g)=N^{-d} \int_{[0, N]^{d}} g^{\prime}(u(t, x)) D_{s, z} u(t, x) \mathrm{d} x .
$$

However, when $g$ goes beyond the globally Lipschitz functions, we need to identify those $g$ 's that satisfy $g(u(t, x)) \in \mathbb{D}^{1, k}$ for all $k \geq 2, t>0$, and $x \in \mathbb{R}^{d}$, in order to apply Clark-Ocone formula (3.3). The following lemma serves this purpose. Indeed, an application of part (i) of Lemma 4.3 below shows that under conditions of part (ii) of Theorem 2.1, $\mathcal{S}_{N, t}(g) \in \mathbb{D}^{1,4} \subseteq \mathbb{D}^{1,2}$.

Lemma 4.3. Let $I=\mathbb{R}$ or $I=(0, \infty)$. Suppose that $F$ is a random variable taking values in $I$ that belongs to $\cap_{k \geq 1} \mathbb{D}^{1, k}$. Let $g: I \rightarrow \mathbb{R}$ be a measurable function such that $\|g(F)\|_{k}<\infty$ for some $k \geq 2$. Then:

(i) If $g \in C^{1}(I)$ satisfies $\left\|g^{\prime}(F)\right\|_{p}<\infty$ for some $p>k$, then $g(F) \in \mathbb{D}^{1, k}$ and

$$
D[g(F)]=g^{\prime}(F) D F .
$$

(ii) If $g \in C^{2}(I)$ satisfies $\left\|g^{\prime}(F)\right\|_{p}+\left\|g^{\prime \prime}(F)\right\|_{p}<\infty$ for some $p>k$, then $g(F) \in \mathbb{D}^{2, k}$ and

$$
D^{2}[g(F)]=g^{\prime \prime}(F) D F \otimes D F+g^{\prime}(F) D^{2} F .
$$

Proof. We prove (i); (ii) is proved similarly and so we skip the proof of that part. For every $M \in \mathbb{N}$ and $x>0$, we set $g_{M}(x)=(g(x) \wedge M) \vee(-M)$. Because $g_{M}$ is Lipschitz, by the chain rule for the Malliavin calculus $g_{M}(F) \in \mathbb{D}^{1, k}$ and

$$
D\left[g_{M}(F)\right]=g^{\prime}(F) \mathbf{1}_{\{|F| \leq M\}} D F \quad \text { a.s. }
$$

Because $\|g(F)\|_{k}<\infty, g_{M}(F)$ converges to $g(F)$ as $M \rightarrow \infty$ in $L^{k}(\Omega)$. Moreover, by Hölder's inequality and (4.4), $D\left[g_{M}(F)\right]$ converges in $L^{k}(\Omega ; \mathcal{H})$ to the right-hand side of $(4.3)$ as $M \rightarrow \infty$. This completes the proof of $(i)$. 
Once the property $\mathcal{S}_{N, t}(g) \in \mathbb{D}^{1,2}$ is established, one can apply the Clark-Ocone formula (3.3) to see that a.s., $\mathcal{S}_{N, t}(g)=\int_{(0, t) \times \mathbb{R}^{d}} \mathrm{E}\left[D_{s, z} \mathcal{S}_{N, t}(g) \mid \mathcal{F}_{s}\right] \eta(\mathrm{d} s \mathrm{~d} z)$. Since the Walsh stochastic integral is an extension of the divergence operator $\delta$, it follows that (a.s.)

$$
\mathcal{S}_{N, t}(g)=\delta\left(v_{N, t}(g)\right) \quad \text { with } \quad v_{N, t}(g)(s, z):=\mathrm{E}\left[D_{s, z} \mathcal{S}_{N, t}(g) \mid \mathcal{F}_{s}\right] \mathbf{1}_{[0, t]}(s) .
$$

Throughout the paper,we denote by

$$
K_{N, t_{1}, t_{2}}:=N^{d}\left\langle D \mathcal{S}_{N, t_{1}}(g), v_{N, t_{2}}(g)\right\rangle_{\mathcal{H}} \quad \text { and } \quad K_{N}:=K_{N, t, t} .
$$

The next lemma shows that properties (2.2) and (2.3) hold under assumptions (ii) or (iii) of Theorem 2.1.

Lemma 4.4. Suppose that either $g \in C^{1}(\mathbb{R})$ or $\sigma(0)=0, g \in C^{1}(0, \infty)$ and $f$ satisfies the reinforced Dalang's condition (1.6) . Suppose, in addition that (2.5) holds for some number $k>2$. Then, (2.3) and (2.2) hold for all $t_{1}, t_{2} \geq 0$.

Proof. Let us first show (2.3). First of all, note that $g(u(t, x)) \in \mathbb{D}^{1,2}$ thanks to Lemma 4.3 and the fact that (2.5) holds for some $k>2$. This proves that the Clark-Ocone formula (3.3) is valid. Applying Clark-Ocone formila yields

$$
\begin{aligned}
\operatorname{Cov}\left[g\left(u\left(t_{1}, x\right)\right), g\left(u\left(t_{2}, 0\right)\right)\right]=\mathrm{E} & \left(\int_{0}^{\infty} \mathrm{d} s \int_{\mathbb{R}^{d}} \mathrm{~d} y \int_{\mathbb{R}^{d}} f(\mathrm{~d} z) \mathrm{E}\left[g^{\prime}\left(u\left(t_{1}, x\right)\right) D_{s, y} u\left(t_{1}, x\right) \mathbf{1}_{\left[0, t_{1}\right]}(s) \mid \mathcal{F}_{s}\right]\right. \\
& \left.\times \mathrm{E}\left[g^{\prime}\left(u\left(t_{2}, 0\right)\right) D_{s, y} u\left(t_{2}, 0\right) \mathbf{1}_{\left[0, t_{2}\right]}(s) \mid \mathcal{F}_{s}\right]\right) .
\end{aligned}
$$

Finally, Hölder's inequality and the estimate (3.9) together yield

$$
\begin{aligned}
\int_{\mathbb{R}^{d}}\left|\operatorname{Cov}\left[g\left(u\left(t_{1}, x\right)\right), g\left(u\left(t_{2}, 0\right)\right)\right]\right| \mathrm{d} x & \leq C \int_{0}^{t_{1} \wedge t_{2}} \mathrm{~d} s \int_{\mathbb{R}^{3 d}} \mathrm{~d} x \mathrm{~d} y f(\mathrm{~d} z) \boldsymbol{p}_{t_{1}-s}(x-y) \boldsymbol{p}_{t_{2}-s}(x-y-z) \\
& =C f\left(\mathbb{R}^{d}\right)\left(t_{1} \wedge t_{2}\right)<\infty .
\end{aligned}
$$

The equality in (2.2) follows from the following claim:

$$
\lim _{N \rightarrow \infty} \operatorname{Cov}\left(N^{d / 2} \mathcal{S}_{N, t_{1}}(g), N^{d / 2} \mathcal{S}_{N, t_{2}}(g)\right)=\int_{\mathbb{R}^{d}} \operatorname{Cov}\left[g\left(u\left(t_{1}, x\right)\right), g\left(u\left(t_{2}, 0\right)\right)\right] \mathrm{d} x .
$$

Indeed, by the stationarity of the solution in its spatial variable, we see that

$$
\begin{aligned}
\operatorname{Cov}\left(N^{d / 2} \mathcal{S}_{N, t_{1}}(g), N^{d / 2} \mathcal{S}_{N, t_{2}}(g)\right) & =\frac{1}{N^{d}} \int_{[0, N]^{d}} \mathrm{~d} x \int_{[0, N]^{d}} \mathrm{~d} y \operatorname{Cov}\left(g\left(u\left(t_{1}, x\right)\right), g\left(u\left(t_{2}, y\right)\right)\right) \\
& =\int_{[0, N]^{d}} \operatorname{Cov}\left(g\left(u\left(t_{1}, z\right)\right), g\left(u\left(t_{2}, 0\right)\right)\right) \mathrm{d} z
\end{aligned}
$$

Now let $N \rightarrow \infty$ to deduce (4.7) from (2.3) and the dominated convergence theorem.

The following proposition is a basic ingredient in the proof of both Theorem 2.1 and 2.3.

Proposition 4.5. Suppose that $f$ satisfies (1.11). Under each of the three cases (i), (ii) and (iii) of Theorem 2.1, the following convergence holds true

$$
\lim _{N \rightarrow \infty} \operatorname{Var}\left(N^{d}\left\langle D \mathcal{S}_{N, t_{1}}(g), v_{N, t_{2}}(g)\right\rangle_{\mathcal{H}}\right)=0, \quad \text { for any } t_{1}, t_{2} \geq 0
$$


Proof of Proposition 4.5 in Case (i). We may use (4.5) together with Fubini's theorem and the definition of $\mathcal{H}$ in order to decompose the quantity of interest $K_{N, t_{1}, t_{2}}$ defined in (4.6) as follows:

$$
\begin{aligned}
K_{N, t_{1}, t_{2}} & =N^{d} \int_{0}^{t_{1} \wedge t_{2}} \mathrm{~d} s \int_{\mathbb{R}^{d}} \mathrm{~d} z D_{s, z} \mathcal{S}_{N, t_{1}}(g)\left(\mathrm{E}\left[D_{s, \bullet} \mathcal{S}_{N, t_{2}}(g) \mid \mathcal{F}_{s}\right] * f\right)(z) \\
& =N^{d} \int_{[0, N]^{d}} \mathrm{~d} x \int_{[0, N]^{d}} \mathrm{~d} y \int_{0}^{t_{1} \wedge t_{2}} \mathrm{~d} s \int_{\mathbb{R}^{d}} \mathrm{~d} z D_{s, z} g\left(u\left(t_{1}, x\right)\right)\left(\mathrm{E}\left[D_{s}, \bullet g\left(u\left(t_{2}, y\right) \mid \mathcal{F}_{s}\right] * f\right)(z)\right. \\
& =I_{1, N}-I_{2, N},
\end{aligned}
$$

where

$$
\begin{aligned}
& I_{1, N}:=N^{d} \int_{[0, N]^{d}} \mathrm{~d} x \int_{\mathbb{R}^{d}} \mathrm{~d} y \int_{0}^{t_{1} \wedge t_{2}} \mathrm{~d} s \int_{\mathbb{R}^{d}} \mathrm{~d} z D_{s, z} g\left(u\left(t_{1}, x\right)\right)\left(\mathrm{E}\left[D_{s, \bullet} g\left(u\left(t_{2}, y\right) \mid \mathcal{F}_{s}\right] * f\right)(z),\right. \\
& I_{2, N}:=N^{d} \int_{[0, N]^{d}} \mathrm{~d} x \int_{\mathbb{R}^{d} \backslash[0, N]^{d}} \mathrm{~d} y \int_{0}^{t_{1} \wedge t_{2}} \mathrm{~d} s \int_{\mathbb{R}^{d}} \mathrm{~d} z D_{s, z} g\left(u\left(t_{1}, x\right)\right)\left(\mathrm{E}\left[D_{s, \bullet} g\left(u\left(t_{2}, y\right) \mid \mathcal{F}_{s}\right] * f\right)(z) .\right.
\end{aligned}
$$

The following shows that the asymptotic behavior of $K_{N, t_{1}, t_{2}}$ is the same as that of $I_{1, N}$.

Claim 1. $I_{2, N} \rightarrow 0$ in $L^{2}(\Omega)$ as $N \rightarrow \infty$. Thus, (4.8) holds if $I_{1, N}-\mathrm{E} I_{1, N} \rightarrow 0$ in $L^{2}(\Omega)$ as $N \rightarrow \infty$.

Proof of Claim 1. The second assertion of Claim 1 is a ready consequence of the first assertion and Jensen's inequality. It suffices to prove the first assertion then. We may appeal to (3.9) with $c=C_{t_{1}, k, \varepsilon, \sigma} C_{t_{2}, k, \varepsilon, \sigma}, k=4$, and $\varepsilon=1 / 2$ (say) as follows:

$$
\begin{aligned}
\left\|I_{2, N}\right\|_{2} & \leq N^{-d} \int_{[0, N]^{d}} \mathrm{~d} x \int_{\mathbb{R}^{d} \backslash[0, N]^{d}} \mathrm{~d} y \int_{0}^{t_{1} \wedge t_{2}} \mathrm{~d} s \int_{\mathbb{R}^{d}} \mathrm{~d} z\left\|D_{s, z} g\left(u\left(t_{1}, x\right)\right)\right\|_{4}\left(\| D_{s, \bullet} g\left(u\left(t_{2}, y\right) \|_{4} * f\right)(z)\right. \\
& \leq \frac{c[\operatorname{Lip}(g)]^{2}}{N^{d}} \int_{[0, N]^{d}} \mathrm{~d} x \int_{\mathbb{R}^{d} \backslash[0, N]^{d}} \mathrm{~d} y \int_{0}^{t_{1} \wedge t_{2}} \mathrm{~d} s \int_{\mathbb{R}^{d}} \mathrm{~d} z \boldsymbol{p}_{t_{1}-s}(x-z)\left(\boldsymbol{p}_{t_{2}-s} * f\right)(z-y) \\
& =\frac{c[\operatorname{Lip}(g)]^{2}}{N^{d}} \int_{[0, N]^{d}} \mathrm{~d} x \int_{\mathbb{R}^{d}} \mathrm{~d} y \int_{0}^{t_{1} \wedge t_{2}} \mathrm{~d} s \mathbf{1}_{\mathbb{R}^{d} \backslash[0, N]^{d}}(y)\left(\boldsymbol{p}_{t_{1}+t_{2}-2 s} * f\right)(x-y) \\
& =c[\operatorname{Lip}(g)]^{2} \int_{0}^{t_{1} \wedge t_{2}} \mathrm{~d} s \int_{\mathbb{R}^{d}} \mathrm{~d} z\left(\boldsymbol{p}_{t_{1}+t_{2}-2 s} * f\right)(z) \int_{\mathbb{R}^{d}} \mathrm{~d} x \mathbf{1}_{[0,1]^{d} \cap\left(\mathbb{R}^{d} \backslash[0,1]^{d}+\frac{z}{N}\right)}(x),
\end{aligned}
$$

owing to the semigroup property of the heat kernel. Now $f$ is a finite measure [see (1.11)] and $\int_{\mathbb{R}^{d}} \mathbf{1}_{[0,1]^{d} \cap\left(\mathbb{R}^{d} \backslash[0,1]^{d}+\frac{z}{N}\right)}(x) \mathrm{d} x \rightarrow 0$ as $N \rightarrow \infty$ for every $z \in \mathbb{R}^{d}$. Therefore, the dominated convergence theorem ensures that the preceding displayed expression converges to zero as $N \rightarrow \infty$. This proves Claim 1.

In order to investigate the asymptotic behavior of $I_{1, N}$, we first define

$$
H_{s}(z):=\int_{\mathbb{R}^{d}} \mathrm{E}\left[D_{s, z} g\left(u\left(t_{2}, \alpha\right)\right) \mid \mathcal{F}_{s}\right] \mathrm{d} \alpha \quad \text { for all } s \in\left[0, t_{2}\right] \text { and } z \in \mathbb{R}^{d} .
$$

Since $D_{s, z} g\left(u\left(t_{2}, y\right)\right)=g^{\prime}\left(u\left(t_{2}, y\right)\right) D_{s, z} u\left(t_{2}, y\right)$ for a.e. $(s, z) \in\left(0, t_{2}\right) \times \mathbb{R}^{d}$, and since $y \mapsto$ $D_{s, z} u\left(t_{2}, y\right)$ is continuous in $L^{2}(\Omega)$ for a.e. $(s, z) \in\left(0, t_{2}\right) \times \mathbb{R}^{d}$ (this can be proved by the same arguments as in [15, Lemma 19]), Lemma 3.3 (applied with $\varepsilon=1 / 2$, say) implies that $H:=\left\{H_{s}(z)\right\}_{(s, z) \in[0, t] \times \mathbb{R}^{d}}$ is an adapted random field that satisfies

$$
\left\|H_{s, z}\right\|_{k} \leq C_{t_{2}, k, 1 / 2, \sigma} \operatorname{Lip}(g) \int_{\mathbb{R}^{d}} \boldsymbol{p}_{t_{2}-s}(y-z) \mathrm{d} y=C_{t_{2}, k, 1 / 2, \sigma} \operatorname{Lip}(g),
$$


uniformly for all $k \geq 2, s \in\left(0, t_{2}\right)$, and $z \in \mathbb{R}^{d}$. Our interest in the random field $H$ stems from the fact that $I_{1, N}$ can be written in terms of $H$ as follows:

$$
I_{1, N}=N^{-d} \int_{[0, N]^{d}} \mathrm{~d} x \int_{0}^{t_{1} \wedge t_{2}} \mathrm{~d} s \int_{\mathbb{R}^{d}} \mathrm{~d} z D_{s, z} g\left(u\left(t_{1}, x\right)\right)\left(H_{s} * f\right)(z)=N^{-d} \int_{[0, N]^{d}} \Psi(x) \mathrm{d} x,
$$

where

$$
\Psi(x):=\int_{0}^{t_{1} \wedge t_{2}} \mathrm{~d} s \int_{\mathbb{R}^{d}} \mathrm{~d} z D_{s, z} g\left(u\left(t_{1}, x\right)\right)\left(H_{s} * f\right)(z) .
$$

Similar estimates to those used in the proof of [7, Theorem 6.4] and [8, Lemma 4.2] show that $(s, z) \mapsto H_{s}(z)$ has a version that is continuous in probability on $\left[t_{1} \wedge t_{2}\right] \times \mathbb{R}^{d}$. In particular, there also exists a measurable version of the random field $\Psi$, and it can be written as $\Psi(x)=g^{\prime}\left(u\left(t_{1}, x\right)\right) \Psi_{1}(x)$, where $x \mapsto \Psi_{1}(x):=\int_{0}^{t_{1}} \mathrm{~d} s \int_{\mathbb{R}^{d}} \mathrm{~d} z D_{s, z} u\left(t_{1}, x\right)\left(H_{s} * f\right)(z)$ is continuous in probability. This and a simple extension of Doob's separability theorem [19] together imply that $H, \Psi_{1}$, and $\Psi$ all have Lebesgue measurable versions, which we feel free to use.

Claim 2. For every $x, y \in \mathbb{R}^{d}, \Psi(x+y)=\Psi(x) \circ \theta_{y}$ a.s.

Proof of Claim 2. We may write

$$
\begin{aligned}
\Psi(x+y) & =\int_{0}^{t_{1} \wedge t_{2}} \mathrm{~d} s \int_{\mathbb{R}^{d}} \mathrm{~d} \alpha \int_{\mathbb{R}^{d}} \mathrm{~d} z D_{s, z} g\left(u\left(t_{1}, x+y\right)\right)\left(\mathrm{E}\left[D_{s, \bullet} g\left(u\left(t_{2}, \alpha\right)\right) \mid \mathcal{F}_{s}\right] * f\right)(z) \\
& =\int_{0}^{t_{1} \wedge t_{2}} \mathrm{~d} s \int_{\mathbb{R}^{d}} \mathrm{~d} \alpha \int_{\mathbb{R}^{d}} \mathrm{~d} z D_{s, z} g\left(u\left(t_{1}, x+y\right)\right)\left(\mathrm{E}\left[D_{s, \bullet} g\left(u\left(t_{2}, \alpha+y\right)\right) \mid \mathcal{F}_{s}\right] * f\right)(z) .
\end{aligned}
$$

We have proven in [7, Lemma 7.1] that for all $t \geq 0, u(t, x+y)=u(t, x) \circ \theta_{y}$ a.s. Therefore, by Lemma 3.5,

$$
\begin{aligned}
\Psi(x+y)= & \int_{0}^{t_{1} \wedge t_{2}} \mathrm{~d} s \int_{\mathbb{R}^{d}} \mathrm{~d} \alpha \int_{\mathbb{R}^{d}} \mathrm{~d} z\left(D_{s, z-y} g\left(u\left(t_{1}, x\right)\right)\right) \circ \theta_{y} \\
& \times\left(\mathrm{E}\left[\left\{D_{s, \bullet-y} g\left(u\left(t_{2}, \alpha\right)\right)\right\} \circ \theta_{y} \mid \mathcal{F}_{s}\right] * f\right)(z) \\
= & \int_{0}^{t_{1} \wedge t_{2}} \mathrm{~d} s \int_{\mathbb{R}^{d}} \mathrm{~d} \alpha \int_{\mathbb{R}^{d}} \mathrm{~d} z\left(D_{s, z} g\left(u\left(t_{1}, x\right)\right)\right) \circ \theta_{y}\left(\left\{\mathrm{E}\left[D_{s, \bullet} g\left(u\left(t_{2}, \alpha\right)\right)\right\} \circ \theta_{y} \mid \mathcal{F}_{s}\right] * f\right)(z),
\end{aligned}
$$

after a change of variables $[z-y \leftrightarrow z]$. Now, $\mathcal{F}_{s}$ is generated by all random variables of the form $\int_{(0, t) \times \mathbb{R}^{d}} \psi(s, x) \eta_{y}(\mathrm{~d} s \mathrm{~d} x)$ as $\psi$ ranges over $\mathcal{H}^{5}$ Because of this observation, it is now a simple exercise in measure theory to verify that $\left\{\mathrm{E}\left[D_{s, \bullet} g\left(u\left(t_{2}, \alpha\right)\right)\right\} \circ \theta_{y} \mid \mathcal{F}_{s}\right]=\mathrm{E}\left[D_{s, \bullet} g\left(u\left(t_{2}, \alpha\right)\right) \mid \mathcal{F}_{s}\right] \circ \theta_{y}$ a.s., which in turn implies that

$$
\Psi(x+y)=\left(\int_{0}^{t_{1} \wedge t_{2}} \mathrm{~d} s \int_{\mathbb{R}^{d}} \mathrm{~d} \alpha \int_{\mathbb{R}^{d}} \mathrm{~d} z D_{s, z} g\left(u\left(t_{1}, x\right)\right)\left(\mathrm{E}\left[D_{s, \bullet} g\left(u\left(t_{2}, \alpha\right)\right) \mid \mathcal{F}_{s}\right] * f\right)(z)\right) \circ \theta_{y} .
$$

almost surely. This proves Claim 2 .

Claim 3. $\Psi(0) \in L^{2}(\Omega)$.

\footnotetext{
${ }^{5}$ Point being that $\mathcal{F}_{s}$ is generated equally well by the infinite-dimensional Brownian motion $\left\{W_{t} \circ \theta_{y}\right\}_{t \geq 0}$ for any $y \in \mathbb{R}^{d}$; compare with (1.4).
} 
Proof of Claim 3. We proceed as we did for Claim 1. Thanks to the definition (4.11) of $\Psi$ and Lemma 3.3,

$$
\begin{aligned}
\|\Psi(0)\|_{2} & \leq C_{t_{1}, 4,1 / 2, \sigma} \operatorname{Lip}(g) f\left(\mathbb{R}^{d}\right) \int_{0}^{t_{1} \wedge t_{2}} \mathrm{~d} s \int_{\mathbb{R}^{d}} \mathrm{~d} z\left\|D_{s, z} g\left(u\left(t_{2}, 0\right)\right)\right\|_{4} \\
& \leq C_{t_{1}, 4,1 / 2, \sigma}[\operatorname{Lip}(g)]^{2} f\left(\mathbb{R}^{d}\right) \int_{0}^{t_{1} \wedge t_{2}} \mathrm{~d} s \int_{\mathbb{R}^{d}} \mathrm{~d} z\left\|D_{s, z} u\left(t_{2}, 0\right)\right\|_{4} \\
& \leq C_{t_{1}, 4,1 / 2, \sigma} C_{t_{2}, 4,1 / 2, \sigma}[\operatorname{Lip}(g)]^{2} f\left(\mathbb{R}^{d}\right)\left(t_{1} \wedge t_{2}\right),
\end{aligned}
$$

where we used (4.9) in the first line (with $k=4$ ), the chain rule of Malliavin calculus (see Nualart [32, Proposition 1.2.3]) in the second line, and Lemma 3.3 in the last line. This verifies Claim 3.

We can now complete the proof of the proposition in Case (i) as follows. Thanks to Claims 2 and 3, we may deduce from Theorem 3.6 that the $L^{2}(\Omega)$-random field $\{\Psi(x)\}_{x \in \mathbb{R}^{d}}$ is stationary and ergodic. Thus, (4.10) and the ergodic theorem together ensure that $I_{1, N} \rightarrow \mathrm{E} \Psi(0)$ as $N \rightarrow \infty$, a.s. and in $L^{2}(\Omega)$. This implies, among other things, that $I_{1, N}-\mathrm{E} I_{1, N} \rightarrow 0$ in $L^{2}(\Omega)$ as $N \rightarrow \infty$. Now apply Claim 1 to deduce (4.8) and hence Case (i) of Proposition 4.5.

Proof of Proposition 4.5 in Cases (ii) and (iii). Cases (ii) and (iii) can be proved similarly. For Claim 1, notice that for $i=1,2$,

$$
\left\|D_{s, z} g\left(u\left(t_{i}, x\right)\right)\right\|_{4} \leq\left\|g^{\prime}\left(u\left(t_{i}, x\right)\right)\right\|_{p}\left\|D_{s, z} u\left(t_{i}, x\right)\right\|_{p^{\prime}}=\left\|g^{\prime}\left(u\left(t_{i}, 0\right)\right)\right\|_{p}\left\|D_{s, z} u\left(t_{i}, x\right)\right\|_{p^{\prime}}
$$

where $p^{-1}+\left(p^{\prime}\right)^{-1}=1 / 4$, and the last equality is due to the stationarity of $x \mapsto u\left(t_{i}, x\right)$. Hence, the moment bound for $I_{2, N}$ is still valid with the constant $c[\operatorname{Lip}(g)]^{2}=C_{t_{1}, 4,1 / 2, \sigma} C_{t_{2}, 4,1 / 2, \sigma}[\operatorname{Lip}(g)]^{2}$ replaced by

$$
C_{t_{1}, p^{\prime}, 1 / 2, \sigma} C_{t_{2}, p^{\prime}, 1 / 2, \sigma}\left\|g^{\prime}\left(u\left(t_{1}, 0\right)\right)\right\|_{p}\left\|g^{\prime}\left(u\left(t_{2}, 0\right)\right)\right\|_{p} .
$$

The above constant is finite thanks to (2.5). Hence, Claim 1 is true. Claim 2 still holds without any change. As for Claim 3, the moment bounds for $\Psi(0)$ are replaced with the following, using similar arguments as above:

$$
\|\Psi(0)\|_{2} \leq C_{t_{1}, p^{\prime}, 1 / 2, \sigma} C_{t_{2}, p^{\prime}, 1 / 2, \sigma}\left\|g^{\prime}\left(u\left(t_{1}, 0\right)\right)\right\|_{p}\left\|g^{\prime}\left(u\left(t_{2}, 0\right)\right)\right\|_{p} f\left(\mathbb{R}^{d}\right)\left(t_{1} \wedge t_{2}\right) .
$$

With these changes, the proof of Cases (ii) and (iii) follows the same arguments as Case (i).

We now proceed with the following.

Proof of Theorem 2.1. We first consider Case (i). Throughout, we choose and fix some $t>0$ and recall that $\lim _{N \rightarrow \infty} \mathbf{B}_{N, t}(g)=\mathbf{B}_{t}(g)>0$. In particular, we observe that $\mathbf{B}_{N, t}(g)>0$ for all sufficiently large $N$. Also, Remark 4.2 shows that $\sqrt{\mathbf{B}_{N, t}(g)} Z$ converges in total variation to $\sqrt{\mathbf{B}_{t}(g)} Z$ as $N \rightarrow \infty$. Therefore, it suffices to prove that

$$
\mathscr{E}_{N}:=d_{\mathrm{TV}}\left(N^{d / 2} \mathcal{S}_{N, t}(g), \sqrt{\mathbf{B}_{N, t}(g)} Z\right) \rightarrow 0 \quad \text { as } N \rightarrow \infty
$$

In view of the representation of $\mathcal{S}_{N, t}(g)$ given in (4.5), we may therefore apply (3.5) to see that

$$
\mathscr{E}_{N} \leq \frac{2}{\mathbf{B}_{N, t}(g)} \mathrm{E}\left(\left|\mathbf{B}_{N, t}(g)-N^{d}\left\langle D \mathcal{S}_{N, t}(g), v_{N, t}(g)\right\rangle_{\mathcal{H}}\right|\right)
$$


valid for all $N$ large enough to ensure that $\mathbf{B}_{N, t}(g)>0$. According to (3.6), $\mathrm{E}\left(\left\langle D \mathcal{S}_{N, t}(g), v_{N, t}(g)\right\rangle_{\mathcal{H}}\right)=$ $\mathbf{B}_{t, N}(g)$. Therefore, in light of (4.12) and (4.13), and owing to Jensen's inequality, it suffices to prove that the variance of $N^{d}\left\langle D \mathcal{S}_{N, t}(g), v_{N, t}(g)\right\rangle_{\mathcal{H}}$ converges to zero as $N \rightarrow \infty$, which has been shown in Proposition 4.5.

For Cases (ii) adn (iii), the limit (2.6) can be proved in the same way as above and (2.3) and (2.2) hold due to Lemma 4.4. Finally, Property $\mathbf{B}_{t}(g)>0$ in Case (iii) is a direct consequence of Corollary 4.7 below and (2.3)

Proposition 4.6 below is devoted to establish the nondegeneracy of $\mathrm{B}_{t}(g)$ in Case (iii) of Theorem 2.1. Our next result is an application of Lemma 3.2 and the Clark-Ocone formula (3.3). In some sense, the following says that the solution $u$ to (1.1) has a strict association property; see Appendix for more exploration in this direction.

Proposition 4.6. Let $u$ denote the solution to (1.1) subject to $u(0) \equiv 1$, and suppose that $\sigma(0)=0$, $\sigma(x) \neq 0$ for all $x>0$, and $f$ satisfies the reinforced Dalang's condition (1.6) for some $\alpha \in(0,1]$. Choose and fix $n$ space-time points $\left(t_{1}, x_{1}\right), \ldots,\left(t_{n}, x_{n}\right) \in(0, \infty) \times \mathbb{R}^{d}$, and real-valued functions $g_{1}, g_{2} \in C^{1}\left((0, \infty)^{n}\right)$ such that for every $i=1, \ldots, n$ and $j=1,2$ :

1. $\partial_{i} g_{j}>0 ;$ and

2. $g_{j}\left(u\left(t_{1}, x_{1}\right), \ldots, u\left(t_{n}, x_{n}\right)\right) \in L^{2}(\Omega)$ and $\partial_{i} g_{j}\left(u\left(t_{1}, x_{1}\right), \ldots, u\left(t_{n}, x_{n}\right)\right) \in \cup_{p>2} L^{p}(\Omega)$.

Then, we have the strict positivity result,

$$
\operatorname{Cov}\left[g_{1}\left(u\left(t_{1}, x_{1}\right), \ldots, u\left(t_{n}, x_{n}\right)\right), g_{2}\left(u\left(t_{1}, x_{1}\right), \ldots, u\left(t_{n}, x_{n}\right)\right)\right]>0 .
$$

Proof. Denote the covariance in (4.14) by $I$. According to Lemma 4.3, Assumption 2 ensures that $g_{j}\left(u\left(t_{1}, x_{1}\right), \ldots, u\left(t_{n}, x_{n}\right)\right) \in \mathbb{D}^{1,2}$ for $j=1,2$. Therefore, we may appeal to the Clark-Ocone formula (3.3) in order to obtain

$$
\begin{aligned}
& I=\mathrm{E}( \int_{0}^{\infty} \mathrm{d} s \int_{\mathbb{R}^{d}} \mathrm{~d} y \int_{\mathbb{R}^{d}} f(\mathrm{~d} z) \\
& \times \mathrm{E} {\left[\sum_{i=1}^{n} \partial_{i} g_{1}\left(u\left(t_{1}, x_{1}\right), \ldots, u\left(t_{n}, x_{n}\right)\right) D_{s, y} u\left(t_{i}, x_{i}\right) \mathbf{1}_{\left[0, t_{i}\right]}(s) \mid \mathcal{F}_{s}\right] } \\
&\left.\times \mathrm{E}\left[\sum_{j=1}^{n} \partial_{j} g_{2}\left(u\left(t_{1}, x_{1}\right), \ldots, u\left(t_{n}, x_{n}\right)\right) D_{s, y+z} u\left(t_{j}, x_{j}\right) \mathbf{1}_{\left[0, t_{j}\right]}(s) \mid \mathcal{F}_{s}\right]\right) .
\end{aligned}
$$

The integral $I$ is well defined and finite. Indeed, Assumption 2, Hölder's inequality and the estimate (3.9) together yield

$$
\begin{aligned}
|I| & \leq C \int_{0}^{\infty} \mathrm{d} s \int_{\mathbb{R}^{d}} \mathrm{~d} y \int_{\mathbb{R}^{d}} f(\mathrm{~d} z)\left(\sum_{i=1}^{n} \boldsymbol{p}_{t_{i}-s}\left(x_{i}-y\right) \mathbf{1}_{\left[0, t_{i}\right]}(s)\right)\left(\sum_{j=1}^{n} \boldsymbol{p}_{t_{j}-s}\left(x_{j}-y-z\right) \mathbf{1}_{\left[0, t_{j}\right]}(s)\right) \\
& \leq C \sum_{i, j=1}^{n} \int_{0}^{t_{i} \wedge t_{j}} \mathrm{~d} s \int_{\mathbb{R}^{d}} \mathrm{~d} y \int_{\mathbb{R}^{d}} f(\mathrm{~d} z) \boldsymbol{p}_{t_{i}-s}\left(x_{i}-y\right) \boldsymbol{p}_{t_{j}-s}\left(x_{j}-y-z\right)<\infty .
\end{aligned}
$$

Since $\sigma(0)=0, u(0) \equiv 1$, and $\sigma(1) \neq 0$, and because $f$ satisfies the reinforced Dalang's condition (1.6) for some $\alpha \in(0,1]$, one can apply Theorem 1.6 of Chen and Huang [5] in order to see that $u(t, x)>0$ almost surely for all $t>0$ and $x \in \mathbb{R}^{d}$. Hence, by Assumption 1 and Theorem 3.2, the two conditional expectations in (4.15) are strictly positive a.s. This proves that $I>0$, which is another way to state the proposition. 
Notice that Proposition 4.6 remains valid if in its assumption 1 we require that all partial derivatives are strictly negative, instead of strictly positive. In this way, we can include examples such as $g\left(x_{1}, \ldots, x_{n}\right)=\prod_{i=1}^{n} x_{i}^{\alpha_{i}}$ where $\alpha_{1}, \ldots, \alpha_{n}<0$.

The following corollary is an immediate consequence of Proposition 4.6.

Corollary 4.7. Let $u$ denote the solution to (1.1) subject to $u(0) \equiv 1$ and $\sigma(0)=0$, and suppose that $f$ satisfies both condition (1.11) and the reinforced Dalang's condition (1.6) for some $\alpha \in(0,1]$. Choose and fix some $g \in C^{1}(0, \infty)$ that satisfies $(2.5)$ for some number $k>2$. Suppose, in addition:

1. $\sigma(x) \neq 0$ for all $x>0$; and

2. $g^{\prime}$ is either strictly positive or strictly negative on $(0, \infty)$;

then $\mathbf{B}_{t_{1}, t_{2}}(g)>0$ for all $t_{1}, t_{2}>0$.

Note that all examples in (1.12) satisfy the conditions of the above corollary.

\section{Functional CLT: Proof of Theorem 2.3}

In order to show Theorem 2.3 we will establish the weak convergence of the finite-dimensional distributions, as well as tightness. We start with a couple of technical lemmas.

Lemma 5.1. Fix $0 \leq a<b \leq \tau, k \geq 2$. Let $\{\Phi(s, y)\}_{(s, y) \in[0, T] \times \mathbb{R}^{d}}$ be an adapted and measurable random field such that, for some constants $M \geq 1$ and $K>0$, and for some function $\varphi:[0, \tau] \rightarrow$ $\mathbb{R}_{+}$,

$$
\|\Phi(s, y)\|_{k} \leq K N^{-d} \varphi(s) \int_{[0, N]^{d}} \boldsymbol{p}_{M(\tau-s)}(x-y) \mathrm{d} x,
$$

for all $(s, y) \in[0, \tau] \times \mathbb{R}^{d}$. Then,

$$
\left\|\int_{[a, b] \times \mathbb{R}^{d}} \Phi(s, y) \eta(\mathrm{d} s \mathrm{~d} y)\right\|_{k} \leq 2 \sqrt{k f\left(\mathbb{R}^{d}\right)} K N^{-d / 2}\left(\int_{a}^{b} \varphi^{2}(s) \mathrm{d} s\right)^{1 / 2} .
$$

Proof. By the BDG inequality, the estimate (5.1) and the semigroup property of the heat kernel together yield

$$
\begin{aligned}
& \left\|\int_{[a, b] \times \mathbb{R}^{d}} \Phi(s, y) \eta(\mathrm{d} s \mathrm{~d} y)\right\|_{k}^{2} \leq 4 k \int_{a}^{b} \mathrm{~d} s \int_{\mathbb{R}^{2 d}} \mathrm{~d} y f(\mathrm{~d} z)\|\Phi(s, y)\|_{k}\|\Phi(s, y-z)\|_{k} \\
& \quad \leq 4 k K^{2} N^{-2 d} \int_{[0, N]^{2 d}} \mathrm{~d} x_{1} \mathrm{~d} x_{2} \int_{a}^{b} \mathrm{~d} s \varphi^{2}(s) \int_{\mathbb{R}^{2 d}} \mathrm{~d} y f(\mathrm{~d} z) \boldsymbol{p}_{M(\tau-s)}\left(x_{1}-y\right) \boldsymbol{p}_{M(\tau-s)}\left(x_{2}-y+z\right) \\
& \quad=4 k K^{2} N^{-2 d} \int_{[0, N]^{2 d}} \mathrm{~d} x_{1} \mathrm{~d} x_{2} \int_{a}^{b} \mathrm{~d} s \varphi^{2}(s) \int_{\mathbb{R}^{d}} f(\mathrm{~d} z) \boldsymbol{p}_{2 M(\tau-s)}\left(x_{1}-x_{2}-z\right) \\
& \leq 4 k K^{2} N^{-d} f\left(\mathbb{R}^{d}\right) \int_{a}^{b} \varphi^{2}(s) \mathrm{d} s .
\end{aligned}
$$

The proof is complete.

Lemma 5.2. For every $\rho \in[0,1)$ there exists a constant $C_{\rho}$ such that

$$
\int_{0}^{1}(1-x)^{-\rho} \mathrm{e}^{-\lambda x(1-x)} \mathrm{d} x \leq C_{\rho}\left(1 \wedge \lambda^{\rho-1}\right) \quad \text { for every } \lambda>0 .
$$


Proof. Without loss of generality, we may - and will - assume that $\lambda \geq 1$. Now simple computations allow us to write

$$
\begin{aligned}
\int_{0}^{1}(1-x)^{-\rho} \mathrm{e}^{-\lambda x(1-x)} \mathrm{d} x & =\int_{0}^{1 / 2}(1-x)^{-\rho} \mathrm{e}^{-\lambda x(1-x)} \mathrm{d} x+\int_{1 / 2}^{1}(1-x)^{-\rho} \mathrm{e}^{-\lambda x(1-x)} \mathrm{d} x \\
& \leq 2^{\rho} \int_{0}^{1 / 2} \mathrm{e}^{-\lambda x / 2} \mathrm{~d} x+\int_{1 / 2}^{1}(1-x)^{-\rho} \mathrm{e}^{-\lambda(1-x) / 2} \mathrm{~d} x \\
& \leq\left(2^{\rho}+1\right) \int_{0}^{\infty} x^{-\rho} \mathrm{e}^{-\lambda x / 2} \mathrm{~d} x<4 \Gamma(1-\rho) \lambda^{\rho-1} .
\end{aligned}
$$

This provides the desired estimate.

According to Sanz-Solé and Sarrà [37], the reinforced Dalang's condition (1.6) implies the following Hölder continuity of the solution:

$$
\sup _{x \in \mathbb{R}^{d}}\left\|u\left(t_{2}, x\right)-u\left(t_{1}, x\right)\right\|_{p} \leq C_{T, p, \gamma}\left|t_{2}-t_{1}\right|^{\gamma}
$$

valid for all $t_{1}, t_{2} \in[0, T], \gamma \in(0, \alpha / 2)$, and $p \geq 2$. The next proposition provides moment estimates which are well tailored to establish tightness.

Proposition 5.3. Assume that $f$ satisfies both (1.11) and the reinforced Dalang's condition (1.6) for some $\alpha \in(0,1]$. Then we have the following two cases:

(i) If $g \in C^{1}(\mathbb{R})$ is such that $g^{\prime}$ is Hölder continuous of order $\delta \in(0,1]$, then for every $T>0$, $\gamma \in(0, \alpha / 2)$, and $k \geq 2 \vee \delta^{-1}$, there exists a number $L>0$ depending on $T, k, \sigma, f, \gamma$ and $g$ such that

$$
\sup _{0 \leq t_{1}, t_{2} \leq T} \mathrm{E}\left(\left|\mathcal{S}_{N, t_{2}}(g)-\mathcal{S}_{N, t_{1}}(g)\right|^{k}\right) \leq L\left|t_{2}-t_{1}\right|^{k \gamma \delta} N^{-k d / 2} .
$$

(ii) If $\sigma(0)=0$ and if $g \in C^{2}(0, \infty)$ is such that $g^{\prime \prime}$ is monotone over $(0, \infty)$ and condition $(2.10)$ is satisfied, then for every $T>0, \gamma \in(0, \alpha / 2)$ and $k \geq 2$ there exists a number $L>0$ depending on $T, k, \sigma, f, \gamma$ and $g$ such that

$$
\sup _{0 \leq t_{1}, t_{2} \leq T} \mathrm{E}\left(\left|\mathcal{S}_{N, t_{2}}(g)-\mathcal{S}_{N, t_{1}}(g)\right|^{k}\right) \leq L\left|t_{2}-t_{1}\right|^{k \gamma} N^{-k d / 2} .
$$

Proof of Case (i) of Proposition 5.3. Fix $\rho \in(0,1 / 2)$. For all $N>0$ and $0 \leq t_{1} \leq t_{2} \leq T$ we can write using Clark-Ocone formula (3.3) and stochastic Fubini's theorem

$$
\begin{aligned}
\mathcal{S}_{N, t_{2}}(g)-\mathcal{S}_{N, t_{1}}(g) & =N^{-d} \int_{[0, N]^{d}}\left[g\left(u\left(t_{2}, x\right)\right)-\mathrm{E}\left(g\left(u\left(t_{2}, x\right)\right)\right)-g\left(u\left(t_{1}, x\right)\right)+\mathrm{E}\left(g\left(u\left(t_{1}, x\right)\right)\right)\right] \mathrm{d} x \\
& =\int_{\left[0, t_{1}\right] \times \mathbb{R}^{d}} \mathcal{A}(s, y) \eta(\mathrm{d} s \mathrm{~d} y)+\int_{\left[t_{1}, t_{2}\right] \times \mathbb{R}^{d}} \mathcal{B}(s, y) \eta(\mathrm{d} s \mathrm{~d} y),
\end{aligned}
$$

where

$$
\begin{array}{ll}
\mathcal{A}(s, y):=N^{-d} \int_{[0, N]^{d}} \mathrm{E}\left[D_{s, y}\left(g\left(u\left(t_{2}, x\right)\right)-g\left(u\left(t_{1}, x\right)\right)\right) \mid \mathcal{F}_{s}\right] \mathrm{d} x & {\left[0 \leq s \leq t_{1}\right],} \\
\mathcal{B}(s, y):=N^{-d} \int_{[0, N]^{d}} \mathrm{E}\left[D_{s, y} g\left(u\left(t_{2}, x\right)\right) \mid \mathcal{F}_{s}\right] \mathrm{d} x & {\left[t_{1} \leq s \leq t_{2}\right] .}
\end{array}
$$


We can make the additional decomposition, $\mathcal{A}(s, y)=: \mathcal{A}_{1}(s, y)+\mathcal{A}_{2}(s, y)$, where

$$
\begin{aligned}
& \mathcal{A}_{1}(s, y):=N^{-d} \int_{[0, N]^{d}} \mathrm{E}\left[\left(g^{\prime}\left(u\left(t_{2}, x\right)\right)-g^{\prime}\left(u\left(t_{1}, x\right)\right)\right) D_{s, y} u\left(t_{2}, x\right) \mid \mathcal{F}_{s}\right] \mathrm{d} x, \\
& \mathcal{A}_{2}(s, y):=N^{-d} \int_{[0, N]^{d}} \mathrm{E}\left[g^{\prime}\left(u\left(t_{2}, x\right)\right)\left[D_{s, y} u\left(t_{1}, x\right)-D_{s, y} u\left(t_{2}, x\right)\right] \mid \mathcal{F}_{s}\right] \mathrm{d} x .
\end{aligned}
$$

We first estimate the $k$-norm of the random variables $\mathcal{A}_{1}(s, y), \mathcal{A}_{2}(s, y)$, and $\mathcal{B}(s, y)$ in order to apply Lemma 5.1. The Hölder continuity of $g^{\prime}$, the estimate (3.9), and the Hölder continuity of $u(t, x)$ (see (5.2)) together imply

$$
\begin{aligned}
\left\|\mathcal{A}_{1}(s, y)\right\|_{k} & \leq L_{\delta}\left(g^{\prime}\right) \sup _{x \in \mathbb{R}^{d}}\left\|u\left(t_{2}, x\right)-u\left(t_{1}, x\right)\right\|_{2 k \delta^{\delta}}^{\delta} N^{-d} \int_{[0, N]^{d}}\left\|D_{s, y} u\left(t_{2}, x\right)\right\|_{2 k} \mathrm{~d} x \\
& \leq C_{T, 2 k, \varepsilon, \sigma} L_{\delta}\left(g^{\prime}\right) C_{T, 2 k \delta, \gamma}^{\delta}\left|t_{2}-t_{1}\right|^{\gamma \delta} N^{-d} \int_{[0, N]^{d}} \boldsymbol{p}_{t_{2}-s}(x-y) \mathrm{d} x,
\end{aligned}
$$

where $L_{\delta}\left(g^{\prime}\right)=\sup _{-\infty<a<b<\infty}\left|g^{\prime}(b)-g^{\prime}(a)\right| /|b-a|^{\delta}$.

The estimation of the $k$-norm of $\mathcal{A}_{2}(s, y)$ is more involved. We first write for every $s \in\left[0, t_{1}\right]$ and $y \in \mathbb{R}^{d}$,

$$
\begin{aligned}
D_{s, y} u\left(t_{1}, x\right)-D_{s, y} u\left(t_{2}, x\right)= & \sigma(u(s, y))\left[\boldsymbol{p}_{t_{1}-s}(x-y)-\boldsymbol{p}_{t_{2}-s}(x-y)\right] \\
& +\int_{s}^{t_{1}} \int_{\mathbb{R}^{d}}\left[\boldsymbol{p}_{t_{1}-r}(x-z)-\boldsymbol{p}_{t_{2}-r}(x-z)\right] \sigma^{\prime}(u(r, z)) D_{s, y} u(r, z) \eta(\mathrm{d} r \mathrm{~d} z) \\
& +\int_{t_{1}}^{t_{2}} \int_{\mathbb{R}^{d}} \boldsymbol{p}_{t_{2}-r}(x-z) \sigma^{\prime}(u(r, z)) D_{s, y} u(r, z) \eta(\mathrm{d} r \mathrm{~d} z),
\end{aligned}
$$

where $\sigma^{\prime}$ denotes the weak derivative of $\sigma$; see Remark 4.1. As a consequence,

$$
\left\|\mathcal{A}_{2}(s, y)\right\|_{k} \leq N^{-d} \int_{[0, N] d}\left\|g^{\prime}\left(u\left(t_{2}, x\right)\right)\right\|_{2 k}\left[\Phi_{1}(x)+\Phi_{2}(x)+\Phi_{3}(x)\right] \mathrm{d} x,
$$

where

$$
\begin{aligned}
\Phi_{1} & :=\|\sigma(u(s, y))\|_{2 k}\left|\boldsymbol{p}_{t_{1}-s}(x-y)-\boldsymbol{p}_{t_{2}-s}(x-y)\right|, \\
\Phi_{2} & :=\left\|\int_{\left(s, t_{1}\right) \times \mathbb{R}^{d}}\left[\boldsymbol{p}_{t_{1}-r}(x-z)-\boldsymbol{p}_{t_{2}-r}(x-z)\right] \sigma^{\prime}(u(r, z)) D_{s, y} u(r, z) \eta(\mathrm{d} r \mathrm{~d} z)\right\|_{2 k}, \\
\Phi_{3}: & :\left\|\int_{\left(t_{1}, t_{2}\right) \times \mathbb{R}^{d}} \boldsymbol{p}_{t_{2}-r}(x-z) \sigma^{\prime}(u(r, z)) D_{s, y} u(r, z) \eta(\mathrm{d} r \mathrm{~d} z)\right\|_{2 k} .
\end{aligned}
$$

First we estimate $\Phi_{1}$. Let $C_{T, 2 k, \sigma}=\sup _{t \in[0, T]}\|\sigma(u(s, 0))\|_{2 k}$ and use the following estimate from Chen and Huang [5, Lemma 4.1]:

$$
\left|\boldsymbol{p}_{t_{1}-s}(x-y)-\boldsymbol{p}_{t_{2}-s}(x-y)\right| \leq C\left(\frac{t_{2}-t_{1}}{t_{1}-s}\right)^{\rho / 2} \boldsymbol{p}_{4\left(t_{2}-s\right)}(x-y),
$$

valid with $\rho=2 \gamma \delta<\alpha$, where $\alpha \in(0,1]$ is the exponent that appears in the reinforced Dalang's condition (1.6). Thus, we obtain

$$
\Phi_{1} \leq C_{T, 2 k, \sigma} C\left(\frac{t_{2}-t_{1}}{t_{1}-s}\right)^{\gamma \delta} \boldsymbol{p}_{4\left(t_{2}-s\right)}(x-y) .
$$


For $\Phi_{2}$, we apply the BDG inequality, (5.4), and (3.9), and write

$$
\begin{aligned}
\Phi_{2}^{2} \leq & 8 k\left[\operatorname{Lip}(\sigma) C_{T, 2 k, \varepsilon, \sigma}\right]^{2} \int_{s}^{t_{1}} \mathrm{~d} r \int_{\mathbb{R}^{d}} \mathrm{~d} z \int_{\mathbb{R}^{d}} f\left(\mathrm{~d} z^{\prime}\right)\left|\boldsymbol{p}_{t_{1}-r}(x-z)-\boldsymbol{p}_{t_{2}-r}(x-z)\right| \\
& \times\left|\boldsymbol{p}_{t_{1}-r}\left(x-z+z^{\prime}\right)-\boldsymbol{p}_{t_{2}-r}\left(x-z+z^{\prime}\right)\right| \boldsymbol{p}_{r-s}(y-z) \boldsymbol{p}_{r-s}\left(y-z+z^{\prime}\right) \\
\leq & 8 k\left[\operatorname{Lip}(\sigma) C C_{T, 2 k, \varepsilon, \sigma}\right]^{2}\left(t_{2}-t_{1}\right)^{\rho} \int_{s}^{t_{1}} \frac{\mathrm{d} r}{\left(t_{1}-r\right)^{\rho}} \\
& \times \int_{\mathbb{R}^{d}} \mathrm{~d} z \int_{\mathbb{R}^{d}} f\left(\mathrm{~d} z^{\prime}\right) \boldsymbol{p}_{4\left(t_{2}-r\right)}(x-z) \boldsymbol{p}_{4\left(t_{2}-r\right)}\left(x-z+z^{\prime}\right) \boldsymbol{p}_{r-s}(y-z) \boldsymbol{p}_{r-s}\left(y-z+z^{\prime}\right) \\
\leq & 2^{2 d+2} k\left[\operatorname{Lip}(\sigma) C C_{T, 2 k, \varepsilon, \sigma}\right]^{2}\left(t_{2}-t_{1}\right)^{\rho} \int_{s}^{t_{1}} \frac{\mathrm{d} r}{\left(t_{1}-r\right)^{\rho}} \\
& \times \int_{\mathbb{R}^{d}} \mathrm{~d} z \int_{\mathbb{R}^{d}} f\left(\mathrm{~d} z^{\prime}\right) \boldsymbol{p}_{4\left(t_{2}-r\right)}(x-z) \boldsymbol{p}_{4\left(t_{2}-r\right)}\left(x-z+z^{\prime}\right) \boldsymbol{p}_{4(r-s)}(y-z) \boldsymbol{p}_{4(r-s)}\left(y-z+z^{\prime}\right) .
\end{aligned}
$$

The elementary identity,

$$
\boldsymbol{p}_{\sigma}(x) \boldsymbol{p}_{\sigma}(y)=2^{d} \boldsymbol{p}_{2 \sigma}(x-y) \boldsymbol{p}_{2 \sigma}(x+y),
$$

valid for every $\sigma>0$ and $x, y \in \mathbb{R}^{d}$, yields the following with $C^{(1)}:=2^{2 d+2} k\left[\operatorname{Lip}(\sigma) C C_{T, 2 k, \varepsilon, \sigma}\right]^{2}$ :

$$
\begin{aligned}
\Phi_{2}^{2} \leq & C^{(1)}\left(t_{2}-t_{1}\right)^{\rho} \int_{s}^{t_{1}} \frac{\mathrm{d} r}{\left(t_{1}-r\right)^{\rho}} \\
& \times \int_{\mathbb{R}^{d}} \mathrm{~d} z \int_{\mathbb{R}^{d}} f\left(\mathrm{~d} z^{\prime}\right) \boldsymbol{p}_{8\left(t_{2}-r\right)}\left(2 x-2 z+z^{\prime}\right) \boldsymbol{p}_{8\left(t_{2}-r\right)}\left(z^{\prime}\right) \boldsymbol{p}_{8(r-s)}\left(2 y-2 z+z^{\prime}\right) \boldsymbol{p}_{8(r-s)}\left(z^{\prime}\right) \\
= & 2^{-d} C^{(1)}\left(t_{2}-t_{1}\right)^{\rho} \boldsymbol{p}_{8\left(t_{2}-s\right)}(2(x-y)) \int_{s}^{t_{1}} \frac{\mathrm{d} r}{\left(t_{1}-r\right)^{\rho}} \int_{\mathbb{R}^{d}} f\left(\mathrm{~d} z^{\prime}\right) \boldsymbol{p}_{8\left(t_{2}-r\right)}\left(z^{\prime}\right) \boldsymbol{p}_{8(r-s)}\left(z^{\prime}\right) \\
= & 2^{-2 d} C^{(1)}\left(t_{2}-t_{1}\right)^{\rho} \boldsymbol{p}_{4\left(t_{2}-s\right)}^{2}(x-y) \int_{s}^{t_{1}} \frac{\mathrm{d} r}{\left(t_{1}-r\right)^{\rho}} \int_{\mathbb{R}^{d}} f\left(\mathrm{~d} z^{\prime}\right) \boldsymbol{p}_{8\left(t_{2}-r\right)(r-s) /\left(t_{2}-s\right)}\left(z^{\prime}\right) .
\end{aligned}
$$

In the last equality we used the formulas

$$
\boldsymbol{p}_{\sigma}(x) \boldsymbol{p}_{\tau}(x)=(2 \pi)^{-d / 2}(\sigma+\tau)^{-d / 2} \boldsymbol{p}_{\sigma \tau /(\sigma+\tau)}(x) \quad\left[\sigma, \tau>0, x \in \mathbb{R}^{d}\right],
$$

and

$$
\boldsymbol{p}_{\sigma}(2 x)=2^{-d}(2 \pi \sigma)^{d / 2} \boldsymbol{p}_{\sigma / 2}^{2}(x) \quad\left[\sigma>0, x \in \mathbb{R}^{d}\right] .
$$

Plancherel's identity allows us to write

$$
\int_{\mathbb{R}^{d}} p_{8\left(t_{2}-r\right)(r-s) /\left(t_{2}-s\right)}(z) f(\mathrm{~d} z)=(2 \pi)^{-d} \int_{\mathbb{R}^{d}} \exp \left(-\frac{4\left(t_{2}-r\right)(r-s)}{t_{2}-s}\|\xi\|^{2}\right) \hat{f}(\xi) \mathrm{d} \xi .
$$

Moreover,

$$
\begin{gathered}
\int_{s}^{t_{1}} \exp \left(-\frac{4\left(t_{2}-r\right)(r-s)}{t_{2}-s}\|\xi\|^{2}\right) \frac{\mathrm{d} r}{\left(t_{1}-r\right)^{\rho}} \leq \int_{s}^{t_{1}} \exp \left(-\frac{4\left(t_{1}-r\right)(r-s)}{t_{1}-s}\|\xi\|^{2}\right) \frac{\mathrm{d} r}{\left(t_{1}-r\right)^{\rho}} \\
\quad=\left(t_{1}-s\right)^{1-\rho} \int_{0}^{1} \exp \left(-4 x(1-x)\left(t_{1}-s\right)\|\xi\|^{2}\right) \frac{\mathrm{d} x}{(1-x)^{\rho}} \\
\leq C_{\rho}\left(t_{1}-s\right)^{1-\rho}\left(1 \wedge\left(4\left(t_{1}-s\right)\|\xi\|^{2}\right)^{\rho-1}\right) \\
\leq C_{\rho} 4^{\rho-1}\left((4 T)^{1-\rho} \wedge\|\xi\|^{2(\rho-1)}\right),
\end{gathered}
$$


where we made the change of variable $(r-s) /\left(t_{1}-s\right)=x$ in the equality and applied Lemma 5.2 in the second inequality. We substitute (5.10) and (5.11) into (5.7), taking into account that $\int_{\mathbb{R}^{d}}\left((4 T)^{1-\alpha} \wedge\|\xi\|^{2(\rho-1)}\right) \hat{f}(\xi) \mathrm{d} \xi<\infty$ due to (1.6) and $\rho \leq \alpha$, in order to find that

$$
\Phi_{2} \leq L\left(t_{2}-t_{1}\right)^{\rho / 2} \boldsymbol{p}_{4\left(t_{2}-s\right)}(x-y),
$$

where here and for the rest of the proof $L$ will denote a constant that depends on $(T, k, \sigma, \gamma, \rho, f)$, and can vary from line to line. We use the same arguments as before and employ the formulas (5.6), (5.8) and (5.9), in order to write

$$
\begin{aligned}
\Phi_{3}^{2} \leq & 8 k\left[\operatorname{Lip}(\sigma) C_{T, 2 k, \varepsilon, \sigma}\right]^{2} \\
& \times \int_{t_{1}}^{t_{2}} \mathrm{~d} r \int_{\mathbb{R}^{d}} \mathrm{~d} z \int_{\mathbb{R}^{d}} f\left(\mathrm{~d} z^{\prime}\right) \boldsymbol{p}_{t_{2}-r}(x-z) \boldsymbol{p}_{t_{2}-r}\left(x-z+z^{\prime}\right) \boldsymbol{p}_{r-s}(y-z) \boldsymbol{p}_{r-s}\left(y-z+z^{\prime}\right) \\
\leq & 2^{2 d+3} k\left[\operatorname{Lip}(\sigma) C_{T, 2 k, \varepsilon, \sigma}\right]^{2} \\
& \times \int_{t_{1}}^{t_{2}} \mathrm{~d} r \int_{\mathbb{R}^{d}} \mathrm{~d} z \int_{\mathbb{R}^{d}} f\left(\mathrm{~d} z^{\prime}\right) \boldsymbol{p}_{2\left(t_{2}-r\right)}\left(2 x-2 z+z^{\prime}\right) \boldsymbol{p}_{2\left(t_{2}-r\right)}\left(z^{\prime}\right) \boldsymbol{p}_{2(r-s)}\left(2 y-2 z+z^{\prime}\right) \boldsymbol{p}_{2(r-s)}\left(z^{\prime}\right) \\
\leq & C^{(2)} \boldsymbol{p}_{t_{2}-s}^{2}(x-y) \int_{t_{1}}^{t_{2}} \mathrm{~d} r \int_{\mathbb{R}^{d}} \mathrm{~d} z \int_{\mathbb{R}^{d}} f\left(\mathrm{~d} z^{\prime}\right) \boldsymbol{p}_{2\left(t_{2}-r\right)(r-s) /\left(t_{2}-s\right)}\left(z^{\prime}\right),
\end{aligned}
$$

with $C^{(2)}:=8 k\left[\operatorname{Lip}(\sigma) C_{T, 2 k, \varepsilon, \sigma}\right]^{2}$. Again, Plancherel's identity allows us to write

$$
\int_{\mathbb{R}^{d}} p_{2\left(t_{2}-r\right)(r-s) /\left(t_{2}-s\right)}(z) f(\mathrm{~d} z)=(2 \pi)^{-d} \int_{\mathbb{R}^{d}} \exp \left(-\frac{\left(t_{2}-r\right)(r-s)}{t_{2}-s}\|\xi\|^{2}\right) \hat{f}(\xi) \mathrm{d} \xi,
$$

and we have

$$
\begin{aligned}
\int_{t_{1}}^{t_{2}} & \exp \left(-\frac{\left(t_{2}-r\right)(r-s)}{t_{2}-s}\|\xi\|^{2}\right) \mathrm{d} r \leq \int_{t_{1}}^{t_{2}} \exp \left(-\frac{\left(t_{2}-r\right)\left(r-t_{1}\right)}{t_{2}-t_{1}}\|\xi\|^{2}\right) \mathrm{d} r \\
& =\left(t_{2}-t_{1}\right) \int_{0}^{1} \exp \left(-x(1-x)\left(t_{2}-t_{1}\right)\|\xi\|^{2}\right) \mathrm{d} x \\
& \leq C_{0}\left(t_{2}-t_{1}\right)\left(1 \wedge\left(\left(t_{2}-t_{1}\right)\|\xi\|^{2}\right)^{-1}\right) \leq C_{0}\left(t_{2}-t_{1}\right)\left(1 \wedge\left(\left(t_{2}-t_{1}\right)\|\xi\|^{2}\right)^{\alpha-1}\right) \\
& \leq C_{0}\left(t_{2}-t_{1}\right)^{\alpha}\left(T^{1-\alpha} \wedge\|\xi\|^{2 \alpha-1}\right),
\end{aligned}
$$

where we used Lemma 5.2 with $\rho=0$ in the equality. From (5.13), (5.14), and (5.15) we obtain

$$
\Phi_{3} \leq L\left(t_{2}-t_{1}\right)^{\alpha / 2} \boldsymbol{p}_{t_{2}-s}(x-y) .
$$

From (5.5), (5.12) and (5.16) we deduce

$$
\left\|\mathcal{A}_{2}(s, y)\right\|_{k} \leq\left\|g^{\prime}\left(u\left(t_{2}, 0\right)\right)\right\|_{2 k} L\left(t_{2}-t_{1}\right)^{\gamma \delta}\left(t_{1}-s\right)^{-\gamma \delta} N^{-d} \int_{[0, N]^{d}} \boldsymbol{p}_{4\left(t_{2}-s\right)}(x-y) \mathrm{d} x .
$$

The Hölder continuity of $g^{\prime}$ implies that $\sup _{r \in[0, T]}\left\|g^{\prime}(u(r, 0))\right\|_{2 k}<\infty$ and an application of Lemma 5.1 with $[a, b]=\left[0, t_{1}\right], \tau=t_{2}, M=4, \varphi(s)=\left(t_{1}-s\right)^{-\gamma \delta} \mathbf{1}_{\left[0, t_{1}\right)}(s)$ yields the desired bound for $\left\|\int_{\left[0, t_{1}\right] \times \mathbb{R}^{d}} \mathcal{A}(s, y) \eta(\mathrm{d} s \mathrm{~d} y)\right\|_{k}$.

Finally,

$$
\|\mathcal{B}(s, y)\|_{k} \leq \sup _{r \in[0, T]}\left\|g^{\prime}(u(r, 0))\right\|_{2 k} C_{T, 2 k, \sigma, \varepsilon} N^{-d} \int_{[0, N]^{d}} \boldsymbol{p}_{t_{2}-s}(x-y) \mathrm{d} x,
$$

and a further application of Lemma 5.1 with $[a, b]=\left[t_{1}, t_{2}\right], \tau=t_{2}, M=1$, and $\varphi(s)=1$ allows us to bound $\left\|\int_{\left[t_{1}, t_{2}\right] \times \mathbb{R}^{d}} \mathcal{B}(s, y) \eta(\mathrm{d} s \mathrm{~d} y)\right\|_{k}$ by a constant times $N^{-d / 2}\left(t_{2}-t_{1}\right)^{1 / 2}$. The proof of Case (i) of Proposition 5.3 is now complete. 
Proof of Case (ii) of Proposition 5.3. We follow the proof of Case (i) of Proposition 5.3, making necessary changes to adapt the arguments to the present setting. The main difference comes from the bound for $\mathcal{A}_{1}$ under the new assumptions on $g$. In particular, notice that

$$
\begin{aligned}
\left\|g^{\prime}\left(u\left(t_{1}, x\right)\right)-g^{\prime}\left(u\left(t_{2}, x\right)\right)\right\|_{2 k} & \leq\left(\left\|g^{\prime \prime}\left(u\left(t_{1}, x\right)\right)\right\|_{4 k}+\left\|g^{\prime \prime}\left(u\left(t_{2}, x\right)\right)\right\|_{4 k}\right) \sup _{x \in \mathbb{R}^{d}}\left\|u\left(t_{1}, x\right)-u\left(t_{2}, x\right)\right\|_{4 k} \\
& \leq C_{T, 4 k, \gamma}\left(\left\|g^{\prime \prime}\left(u\left(t_{1}, 0\right)\right)\right\|_{4 k}+\left\|g^{\prime \prime}\left(u\left(t_{2}, 0\right)\right)\right\|_{4 k}\right)\left|t_{2}-t_{1}\right|^{\gamma} \\
& \leq C_{T, 4 k, \gamma} \sup _{t \in[0, T]}\left\|g^{\prime \prime}(u(t, 0))\right\|_{4 k}\left|t_{2}-t_{1}\right|^{\gamma} \\
& =: C_{1}\left|t_{2}-t_{1}\right|^{\gamma}
\end{aligned}
$$

where the first inequality is due to the monotonicity of $g^{\prime \prime}$, the second is due to (5.2) and the stationarity of the solution, and the constant $C_{T, 4 k, \gamma}$ comes from (5.2). Note that the continuity of $t \in[0, T] \mapsto\left\|g^{\prime \prime}(u(t, 0))\right\|_{4 k}$ ensures that the above constant $C_{1}$ is finite. Hence,

$$
\left\|\mathcal{A}_{1}(s, y)\right\|_{k} \leq C_{1}\left|t_{1}-t_{2}\right|^{\gamma} N^{-d} \int_{[0, N]^{d}} \boldsymbol{p}_{t_{2}-s}(x-y) \mathrm{d} x
$$

The remainder of the proof is similar, with the remark that $\sup _{r \in[0, T]}\left\|g^{\prime}(u(r, 0))\right\|_{2 k}<\infty$ due to condition (2.10).

Equipped with Proposition 5.3, we can now proceed with the proof of Theorem 2.3.

Proof of Theorem 2.3. We first prove Case (i). Choose and fix some $T>0$. By Proposition 5.3, a standard application of Kolmogorov's continuity theorem and the Arzelà-Ascoli theorem ensures that $\left\{N^{d / 2} \mathcal{S}_{N, \bullet}(g)\right\}_{N \geq 1}$ is a tight net of processes on $C([0, T])$. Therefore, it remains to prove that the finite-dimensional distributions of the process $t \mapsto N^{d / 2} \mathcal{S}_{N, t}(g)$ converge to those of $\left\{\mathcal{G}_{t}\right\}_{t \in[0, T]}$.

Let us choose and fix some $T>0$ and $m \geq 1$ points $t_{1}, \ldots, t_{m} \in(0, T)$. Consider the random vector $F_{N}=\left(F_{N}^{(1)}, \ldots, F_{N}^{(m)}\right)$ defined by

$$
F_{N}^{(i)}:=N^{d / 2} \mathcal{S}_{N, t_{i}}(g), \quad \text { for } i=1, \ldots, m,
$$

let $G=\left(\mathcal{G}_{t_{1}}, \ldots, \mathcal{G}_{t_{m}}\right)$ denote a centered Gaussian random vector with covariance matrix $\left(\mathbf{B}_{t_{i}, t_{j}}(g)\right)_{1 \leq i, j \leq m}$. Recall from (4.5) the random fields $v_{N, t_{1}}(g), \ldots, v_{N, t_{m}}(g)$ which are such that $F_{N}^{(i)}=\delta\left(N^{d / 2} v_{N, t_{i}}(g)\right)$ for all $i=1, \ldots, m$. Set $V_{N}^{(i)}=N^{d / 2} v_{N, t_{i}}(g)$ for $i=1, \ldots, m$ and let $V_{N}=\left(V_{N}^{(1)}, \ldots, V_{N}^{(m)}\right)$. Since $\mathrm{E}\left[F_{N}^{(i)}\right]=0$ and $F_{N}^{(i)} \in \mathbb{D}^{1,4}$ (see (4.1)), we can write, by a generalization of a result of Nourdin and Peccatti [31, Theorem 6.1.2],

$$
\left|\mathrm{E}\left(h\left(F_{N}\right)\right)-\mathrm{E}(h(G))\right| \leq \frac{1}{2}\left\|h^{\prime \prime}\right\|_{\infty} \sqrt{\sum_{i, j=1}^{m} \mathrm{E}\left(\left|\mathbf{B}_{i, j}(g)-\left\langle D F_{N}^{(i)}, V_{N}^{(j)}\right\rangle_{\mathcal{H}}\right|^{2}\right)}
$$

for every $h \in C^{2}\left(\mathbb{R}^{m}\right)$ that has bounded second partial derivatives, and with the notation

$$
\left\|h^{\prime \prime}\right\|_{\infty}=\max _{1 \leq i, j \leq m} \sup _{x \in \mathbb{R}^{m}}\left|\frac{\partial^{2} h(x)}{\partial x_{i} \partial x_{j}}\right| .
$$

Thus, in view of (5.20) in order to show the convergence in law of $F_{N}$ to $G$, it suffices to show that for any $i, j=1, \ldots, m$, we have

$$
\lim _{N \rightarrow \infty} \mathrm{E}\left(\left|\mathbf{B}_{i, j}(g)-\left\langle D F_{N}^{(i)}, V_{N}^{(j)}\right\rangle_{\mathcal{H}}\right|^{2}\right)=0
$$


Notice that, by the duality relationship (3.2) and the convergence (2.2) and we have, as $N \rightarrow \infty$,

$$
\begin{aligned}
\mathrm{E}\left(\left\langle D F_{N}^{(i)}, V_{N}^{(j)}\right\rangle_{\mathcal{H}}\right) & =N^{d} \mathrm{E}\left(\left\langle D \mathcal{S}_{N, t_{i}}(g), v_{N, t_{j}}(g)\right\rangle_{\mathcal{H}}\right) \\
& =N^{d} \mathrm{E}\left(\mathcal{S}_{N, t_{i}}(g), \delta\left(v_{N, t_{j}}(g)\right)\right) \\
& =N^{d} \mathrm{E}\left(\mathcal{S}_{N, t_{i}}(g), \mathcal{S}_{N, t_{j}}(g)\right) \rightarrow \mathbf{B}_{t_{i}, t_{j}}(g) .
\end{aligned}
$$

Therefore, the convergence (5.21) follows immediately from (5.22) and (4.8). It follows from this fact that the finite-dimensional distributions of $t \mapsto N^{d / 2} \mathcal{S}_{N, t}(g)$ converge to those of $\mathcal{G}$ as $N \rightarrow \infty$. The proof of Case (i) of Theorem 2.3 is now complete.

The proof of Case (ii) follows the same lines as that of Case (i) with the following changes: Tightness is a consequence of part (ii) of Proposition 5.3. In order to apply Theorem 6.1.2 of Nourdin and Peccati [31], we need to verify that $\mathcal{S}_{n, t}(g) \in \mathbb{D}^{1,4}$ for all $t>0$. This follows from the fact that $g(u(t, x)) \in \mathbb{D}^{1,4}$ for all $t>0$ and for all $x \in \mathbb{R}^{d}$ due to Lemma 4.3 and assumption (2.10) and the fact that the integral $\int_{[0, N]^{d}} g(u(t, x)) \mathrm{d} x$ can be approximated by Riemann sums in the norm of $\mathbb{D}^{1,4}$. Finally, the convergence in (5.22) is due to (4.7). This completes the proof of Theorem 2.3.

\section{Total Variation Distance for linear $g$ : Proof of Theorem 2.4}

Throughout this section, we choose and fix a number $t>0$. The strategy of the proof of Theorem 2.4 is as follows: We proceed as we did with Theorem 2.1, and begin by appealing to (4.12), (4.5), and (4.13), but use the linearity of $g$ in order to make precise estimates of the centralized moments of $K_{N}$ (rather than appeal to ergodic-theoretic arguments). The details are lengthy and technical, and will follow in due time. For now, we suffice it to say that one can indeed anticipate simplifications in the present setting in which $g$ is linear. For example, because $g(v)=v$ for all $v \in \mathbb{R}^{d}$, (4.2) simplifies to the a.e. identity (in $\left.(s, z) \in(0, t) \times \mathbb{R}^{d}\right)$ :

$$
D_{s, z} \mathcal{S}_{N, t}(g)=N^{-d} \int_{[0, N]^{d}} D_{s, z} u(t, x) \mathrm{d} x
$$

This is one of the quantities that appears in the $\mathcal{H}$-inner product in the definition (4.6) of $K_{N}$. We will be able to make use of the above because of the well-known fact that the integrand $D_{s, z} u(t, x)$ solves the following stochastic integral equation for all $t>0$ and $x \in \mathbb{R}^{d}$ and a.e. $(s, y) \in(0, t) \times \mathbb{R}^{d}$ : a.s.,

$$
D_{s, y} u(t, x)=\boldsymbol{p}_{t-s}(x-y) \sigma(u(s, y))+\int_{(s, t) \times \mathbb{R}^{d}} \boldsymbol{p}_{t-s}(x-z) \sigma^{\prime}(u(r, z)) D_{s, y} u(r, z) \eta(\mathrm{d} r \mathrm{~d} z) .
$$

The above integral eqution also allows us to better understand the second quantity $v_{N, t}(g)$ in the $\mathcal{H}$-inner product of the definition (4.6) of $K_{N}$. Indeed, because of the martingale property of Walsh integral, the above eadily yields the following a.s.-identity that is valid with the same null-set restrictions as does the preceding identity:

$$
\mathrm{E}\left[D_{s, y} u(t, x) \mid \mathcal{F}_{s}\right]=\boldsymbol{p}_{t-s}(x-y) \sigma(u(s, y)) .
$$

Integrate this over $x \in[0, N]^{d}$, apply Fubini's theorem, and divide by $N^{d}$ to find that for all $t>0$ and a.e. $(s, y) \in(0, t) \times \mathbb{R}^{d}$,

$$
\begin{aligned}
& \mathrm{E}\left[D_{s, y} \mathcal{S}_{N, t}(g) \mid \mathcal{F}_{s}\right]=\Pi_{s, y}^{(N)} \sigma(u(s, y)) \quad \text { a.s., where } \\
& \Pi_{s, y}^{(N)}=\Pi_{s, y}^{(N)}(t):=N^{-d} \int_{[0, N]^{d}} \boldsymbol{p}_{t-s}(x-y) \mathrm{d} x .
\end{aligned}
$$


In light of (4.5), we find the following formula for the random field $v_{N, t}(g)$, valid since $g(v)=v$ for all $v \in \mathbb{R}$ : For all $t>0$ and a.e. $(s, z) \in(0, t) \times \mathbb{R}^{d}$,

$$
v_{N, t}(g)(s, z)=\Pi_{s, z}^{(N)} \sigma(u(s, z)) \mathbf{1}_{[0, t]}(s) .
$$

Finally, let us integrate both sides of (6.2) over $x \in[0, N]^{d}$, and then divide by $N^{d}$, in order to deduce from standard Fubini-type arguments and (6.1) that a.s. for a.e. $(s, y) \in(0, t) \times \mathbb{R}^{d}$,

$$
\begin{aligned}
& D_{s, y} \mathcal{S}_{N, t}(g)=v_{N, t}(g)(s, y)+\mathcal{T}(s, y), \quad \text { where } \\
& \mathcal{T}(s, y)=\mathcal{T}_{g, t, N}(s, y):=\int_{(s, t) \times \mathbb{R}^{d}} \Pi_{s, z}^{(N)} \sigma^{\prime}(u(r, z)) D_{s, y} u(r, z) \eta(\mathrm{d} r \mathrm{~d} z)
\end{aligned}
$$

and $\sigma^{\prime}$ is any [measurable] version of the weak derivative of $\sigma$ (see Remark 4.1). And $D_{s, y} \mathcal{S}_{N, t}=0$ when $s \geq t$. The preceding yields, among other things, that

$$
\frac{K_{N}}{N^{d}}=\left\|v_{N, t}(g)\right\|_{\mathcal{H}}^{2}+\left\langle\mathcal{T}, v_{N, t}(g)\right\rangle_{\mathcal{H}}
$$

see (4.6). We estimate the preceding two quantities separately and in turn by way of two lemmas.

Lemma 6.1. There exists a real number $L_{1}=L_{1}\left(f\left(\mathbb{R}^{d}\right)\right.$, Lip $\left.(\sigma)\right)$ such that for every $\varepsilon \in(0,1)$, $N, t>0$,

$$
\operatorname{Var}\left(\left\|v_{N, t}(g)\right\|_{\mathcal{H}}^{2}\right) \leq L_{1} t^{3} C_{t, 4, \varepsilon, \sigma}^{2} A_{t}^{2} N^{-3 d}
$$

where $C_{t, 4, \varepsilon, \sigma}$ was defined in Lemma 3.3 and $A_{t}:=\sup _{s \in(0, t)}\|\sigma(u(s, 0))\|_{4}$.

Proof. Choose and fix $N, t>0$ and $\varepsilon \in(0,1)$ throughout. The definition of the Hilbert space $\mathcal{H}$ implies that

$$
\begin{aligned}
\left\|v_{N, t}(g)\right\|_{\mathcal{H}}^{2} & =\int_{0}^{t} \mathrm{~d} s \int_{\mathbb{R}^{d}} \mathrm{~d} y v_{N, t}(g)(s, y)\left(v_{N, t}(g)(s, \bullet) * f\right)(y) \\
& =\int_{0}^{t} \mathrm{~d} s \int_{\mathbb{R}^{d}} \mathrm{~d} y \int_{\mathbb{R}^{d}} f(\mathrm{~d} z) \Pi_{s, y}^{(N)} \Pi_{s, y-z}^{(N)} \sigma(u(s, y)) \sigma(u(s, y-z)) .
\end{aligned}
$$

Therefore, we can apply Minkowski's inequality for integrals ${ }^{6}$ in order to see that

$$
\begin{aligned}
\operatorname{Var}\left(\left\|v_{N, t}(g)\right\|_{\mathcal{H}}^{2}\right) & \leq\left[\int_{0}^{t}\left\{\operatorname{Var}\left(\int_{\mathbb{R}^{d}} \mathrm{~d} y \int_{\mathbb{R}^{d}} f(\mathrm{~d} z) \Pi_{s, y}^{(N)} \Pi_{s, y-z}^{(N)} \sigma(u(s, y)) \sigma(u(s, y-z))\right)\right\}^{1 / 2} \mathrm{~d} s\right]^{2} \\
& =:\left[\int_{0}^{t}\left\{\operatorname{Var}\left(F_{s}\right)\right\}^{1 / 2} \mathrm{~d} s\right]^{2}
\end{aligned}
$$

the definition of the process $\left\{F_{s}\right\}_{s \in(0, t)}$ being obvious. By the Poincaré inequality (3.4), $\operatorname{Var}\left(F_{s}\right) \leq$ $\mathrm{E}\left(\left\|D F_{s}\right\|_{\mathcal{H}}^{2}\right)$. Therefore, we need to compute $D F_{s}$. But that is, by the chain rule [32, Proposition 1.2 .3 , given by

$$
\begin{aligned}
D_{r, w} F_{s} & =\int_{\mathbb{R}^{d}} \mathrm{~d} y \int_{\mathbb{R}^{d}} f(\mathrm{~d} z) \Pi_{s, y}^{(N)} \Pi_{s, y-z}^{(N)} D_{r, w}[\sigma(u(s, y)) \sigma(u(s, y-z))] \\
& =2 \int_{\mathbb{R}^{d}} \mathrm{~d} y \int_{\mathbb{R}^{d}} f(\mathrm{~d} z) \Pi_{s, y}^{(N)} \Pi_{s, y-z}^{(N)} \sigma^{\prime}(u(s, y)) D_{r, w} u(s, y) \sigma(u(s, y-z)),
\end{aligned}
$$

\footnotetext{
${ }^{6}$ That is, $\left\|\int_{0}^{t}\left[X_{s}-\mathrm{E}\left(X_{s}\right)\right] \mathrm{d} s\right\|_{2} \leq \int_{0}^{t}\left\|X_{s}-\mathrm{E}\left(X_{s}\right)\right\|_{2} \mathrm{~d} s$, whenever this makes sense, for any stochastic process $X=\left\{X_{s}\right\}_{0 \leq s \leq t}$.
} 
where the prefactor 2 comes from symmetry. In this way we find that

$$
\begin{aligned}
\mathrm{E}\left(\left\|D F_{s}\right\|_{\mathcal{H}}^{2}\right)= & \mathrm{E}\left[\int_{0}^{s} \mathrm{~d} r \int_{\mathbb{R}^{d}} \mathrm{~d} w \int_{\mathbb{R}^{d}} f(\mathrm{~d} v) D_{r, w}(F) D_{r, w-v}(F)\right] \\
= & 4 \int_{0}^{s} \mathrm{~d} r \int_{\mathbb{R}^{d}} \mathrm{~d} w \int_{\mathbb{R}^{d}} f(\mathrm{~d} v) \int_{\mathbb{R}^{d}} \mathrm{~d} y \int_{\mathbb{R}^{d}} f(\mathrm{~d} z) \int_{\mathbb{R}^{d}} \mathrm{~d} y^{\prime} \int_{\mathbb{R}^{d}} f\left(\mathrm{~d} z^{\prime}\right) \Pi_{s, y}^{(N)} \Pi_{s, y-z}^{(N)} \Pi_{s, y^{\prime}}^{(N)} \Pi_{s, y^{\prime}-z^{\prime}}^{(N)} \\
& \times \mathrm{E}\left[\sigma^{\prime}(u(s, y)) D_{r, w} u(s, y) \sigma(u(s, y-z)) \sigma^{\prime}\left(u\left(s, y^{\prime}\right)\right) D_{r, w-v} u\left(s, y^{\prime}\right) \sigma\left(u\left(s, y^{\prime}-z^{\prime}\right)\right)\right] .
\end{aligned}
$$

Hölder's inequality ensures that the final expectation in the above display is bounded above in modulus by

$$
\begin{aligned}
& {[\operatorname{Lip}(\sigma)]^{2}\left\|D_{r, w} u(s, y)\right\|_{4}\|\sigma(u(s, y-z))\|_{4}\left\|D_{r, w-v} u\left(s, y^{\prime}\right)\right\|_{4}\left\|\sigma\left(u\left(s, y^{\prime}-z^{\prime}\right)\right)\right\|_{4}} \\
& =[\operatorname{Lip}(\sigma)]^{2}\|\sigma(u(s, 0))\|_{4}^{2}\left\|D_{r, w} u(s, y)\right\|_{4}\left\|D_{r, w-v} u\left(s, y^{\prime}\right)\right\|_{4} \\
& \leq\left[C_{t, 4, \varepsilon, \sigma} A_{t} \operatorname{Lip}(\sigma)\right]^{2} \boldsymbol{p}_{s-r}(y-w) \boldsymbol{p}_{s-r}\left(y^{\prime}-w+v\right),
\end{aligned}
$$

uniformly for all $s \in(0, t)$, where $\varepsilon \in(0,1)$ is arbitrary; see Lemma 3.3 for the last line. These computations and the Poincaré inequality together yield the following:

$$
\begin{aligned}
\operatorname{Var}\left(F_{s}\right) \leq & 4\left[C_{t, 4, \varepsilon, \sigma} A_{t} \operatorname{Lip}(\sigma)\right]^{2} \int_{0}^{s} \mathrm{~d} r \int_{\mathbb{R}^{d}} \mathrm{~d} w \int_{\mathbb{R}^{d}} f(\mathrm{~d} v) \int_{\mathbb{R}^{d}} \mathrm{~d} y \int_{\mathbb{R}^{d}} f(\mathrm{~d} z) \int_{\mathbb{R}^{d}} \mathrm{~d} y^{\prime} \int_{\mathbb{R}^{d}} f\left(\mathrm{~d} z^{\prime}\right) \\
& \times \Pi_{s, y}^{(N)} \Pi_{s, y-z}^{(N)} \Pi_{s, y^{\prime}}^{(N)} \Pi_{s, y^{\prime}-z^{\prime}}^{(N)} \boldsymbol{p}_{s-r}(y-w) \boldsymbol{p}_{s-r}\left(y^{\prime}-w+v\right) \\
= & 4\left[C_{t, 4, \varepsilon, \sigma} A_{t} \operatorname{Lip}(\sigma)\right]^{2} \int_{0}^{s} \mathrm{~d} r \int_{\mathbb{R}^{d}} f(\mathrm{~d} v) \int_{\mathbb{R}^{d}} \mathrm{~d} y \int_{\mathbb{R}^{d}} f(\mathrm{~d} z) \int_{\mathbb{R}^{d}} \mathrm{~d} y^{\prime} \int_{\mathbb{R}^{d}} f\left(\mathrm{~d} z^{\prime}\right) \\
& \times \Pi_{s, y}^{(N)} \Pi_{s, y-z}^{(N)} \Pi_{s, y^{\prime}}^{(N)} \Pi_{s, y^{\prime}-z^{\prime}}^{(N)} \boldsymbol{p}_{2(s-r)}\left(y^{\prime}-y+v\right),
\end{aligned}
$$

owing to the symmetry and semigroup properties of the heat kernel. Since $\Pi^{(N)} \leq N^{-d}$ [see (6.3)] we may replace three of the four terms that involve the $\Pi$ 's with $N^{-d}$ in order to find that

$$
\begin{aligned}
\operatorname{Var}\left(F_{s}\right) \leq & \frac{4\left[C_{t, 4, \varepsilon, \sigma} A_{t} \operatorname{Lip}(\sigma)\right]^{2}}{N^{4 d}} \int_{0}^{s} \mathrm{~d} r \int_{\mathbb{R}^{d}} f(\mathrm{~d} v) \\
& \times \int_{\mathbb{R}^{d}} \mathrm{~d} y \int_{\mathbb{R}^{d}} f(\mathrm{~d} z) \int_{\mathbb{R}^{d}} \mathrm{~d} y^{\prime} \int_{\mathbb{R}^{d}} f\left(\mathrm{~d} z^{\prime}\right) \int_{[0, N]^{d}} \mathrm{~d} a \boldsymbol{p}_{s}(a-y) \boldsymbol{p}_{2(s-r)}\left(y^{\prime}-y+v\right) \\
= & \frac{4\left[C_{t, 4, \varepsilon, \sigma} A_{t} \operatorname{Lip}(\sigma)\right]^{2} f\left(\mathbb{R}^{d}\right)^{2}}{N^{4 d}} \int_{0}^{s} \mathrm{~d} r \int_{\mathbb{R}^{d}} f(\mathrm{~d} v) \int_{\mathbb{R}^{d}} \mathrm{~d} y^{\prime} \int_{[0, N]^{d}} \mathrm{~d} a \boldsymbol{p}_{3 s-2 r}\left(a+y^{\prime}+v\right) \\
= & \frac{4\left[C_{t, 4, \varepsilon, \sigma} A_{t} \operatorname{Lip}(\sigma)\right]^{2} f\left(\mathbb{R}^{d}\right)^{3} s}{N^{4 d}}
\end{aligned}
$$

Plug this bound into (6.6) to deduce the result.

Lemma 6.2. There exists a real number $L_{2}=L_{2}\left(f\left(\mathbb{R}^{d}\right), \operatorname{Lip}(\sigma)\right)$ such that for every $\varepsilon \in(0,1)$, $N, t>0$,

$$
\operatorname{Var}\left(\left\langle\mathcal{T}, v_{N, t}(g)\right\rangle_{\mathcal{H}}\right) \leq L_{2} t^{3} C_{t, 4, \varepsilon, \sigma}^{2} A_{t}^{2} N^{-3 d},
$$

where $C_{t, 4, \varepsilon, \sigma}$ and $A_{t}$ were respectively defined in Lemmas 3.3 and 6.1. 
Proof. Choose and fix $N, t>0$ and $\varepsilon \in(0,1)$ throughout. It follows from (6.4), the definition of the Hilbert space $\mathcal{H}$, and a standard appeal to a stochastic Fubini theorem that

$$
\begin{aligned}
& \left\langle\mathcal{T}, v_{N, t}(g)\right\rangle_{\mathcal{H}}=\int_{0}^{t} \mathrm{~d} s \int_{\mathbb{R}^{d}} \mathrm{~d} y \int_{\mathbb{R}^{d}} f(\mathrm{~d} w) \mathcal{T}(s, y) v_{N, t}(g)(s, y-w) \\
& =\int_{(0, t) \times \mathbb{R}^{d}}\left[\int_{0}^{r} \mathrm{~d} s \int_{\mathbb{R}^{d}} \mathrm{~d} y \int_{\mathbb{R}^{d}} f(\mathrm{~d} w) \Pi_{s, z}^{(N)} \sigma^{\prime}(u(r, z)) D_{s, y} u(r, z) \Pi_{s, y-w}^{(N)} \sigma(u(s, y-w))\right] \eta(\mathrm{d} r \mathrm{~d} z) \\
& =: \int_{(0, t) \times \mathbb{R}^{d}} \mathcal{A}(r, z) \eta(\mathrm{d} r \mathrm{~d} z),
\end{aligned}
$$

notation being clear. Among other things, we see from the above representation that the random variable $\left\langle\mathcal{T}, v_{N, t}(g)\right\rangle_{\mathcal{H}}$ has mean zero. Therefore,

$$
\operatorname{Var}\left(\left\langle\mathcal{T}, v_{N, t}(g)\right\rangle_{\mathcal{H}}\right)=\mathrm{E}\left(\left\langle\mathcal{T}, v_{N, t}(g)\right\rangle_{\mathcal{H}}^{2}\right)=\int_{0}^{t} \mathrm{~d} r \int_{\mathbb{R}^{d}} \mathrm{~d} a \int_{\mathbb{R}^{d}} f(\mathrm{~d} b) \mathrm{E}[\mathcal{A}(r, a) \mathcal{A}(r, a-b)] .
$$

The integrand is bounded as follows:

$$
\begin{aligned}
& \mathrm{E}|\mathcal{A}(r, a) \mathcal{A}(r, a-b)| \\
& \leq \int_{0}^{r} \mathrm{~d} s \int_{\mathbb{R}^{d}} \mathrm{~d} y \int_{\mathbb{R}^{d}} f(\mathrm{~d} w) \int_{0}^{r} \mathrm{~d} s^{\prime} \int_{\mathbb{R}^{d}} \mathrm{~d} y^{\prime} \int_{\mathbb{R}^{d}} f\left(\mathrm{~d} w^{\prime}\right) \Pi_{s, a}^{(N)} \Pi_{s, y-w}^{(N)} \Pi_{s^{\prime}, a-b}^{(N)} \Pi_{s^{\prime}, y^{\prime}-w^{\prime}}^{(N)} \\
& \quad \times \mathrm{E}\left[\left|\sigma^{\prime}(u(r, a)) D_{s, y} u(r, a) \sigma(u(s, y-w))\right|\right. \\
& \left.\quad \times\left|\sigma^{\prime}(u(r, a-b)) D_{s^{\prime}, y^{\prime}} u(r, a-b) \sigma\left(u\left(s^{\prime}, y^{\prime}-w^{\prime}\right)\right)\right|\right] \\
& \leq[\operatorname{Lip}(\sigma)]^{2} \int_{0}^{r} \mathrm{~d} s \int_{\mathbb{R}^{d}} \mathrm{~d} y \int_{\mathbb{R}^{d}} f(\mathrm{~d} w) \int_{0}^{r} \mathrm{~d} s^{\prime} \int_{\mathbb{R}^{d}} \mathrm{~d} y^{\prime} \int_{\mathbb{R}^{d}} f\left(\mathrm{~d} w^{\prime}\right) \Pi_{s, a}^{(N)} \Pi_{s, y-w}^{(N)} \Pi_{s^{\prime}, a-b}^{(N)} \Pi_{s^{\prime}, y^{\prime}-w^{\prime}}^{(N)} \\
& \quad \times\left\|D_{s, y} u(r, a)\right\|_{4}\|\sigma(u(s, y-w))\|_{4}\left\|D_{s^{\prime}, y^{\prime}} u(r, a-b)\right\|_{4}\left\|\sigma\left(u\left(s^{\prime}, y^{\prime}-w^{\prime}\right)\right)\right\|_{4},
\end{aligned}
$$

thanks to Hölder's inequality. Thus, it follows from Lemma 3.3 and the stationarity of $x \mapsto u(s, x)$ that

$$
\begin{aligned}
\mathrm{E}|\mathcal{A}(r, a) \mathcal{A}(r, a-b)| \leq & {\left[A_{t} C_{t, 4, \varepsilon, \sigma} \operatorname{Lip}(\sigma)\right]^{2} \int_{0}^{r} \mathrm{~d} s \int_{\mathbb{R}^{d}} \mathrm{~d} y \int_{\mathbb{R}^{d}} f(\mathrm{~d} w) \int_{0}^{r} \mathrm{~d} s^{\prime} \int_{\mathbb{R}^{d}} \mathrm{~d} y^{\prime} \int_{\mathbb{R}^{d}} f\left(\mathrm{~d} w^{\prime}\right) } \\
& \times \Pi_{s, a}^{(N)} \Pi_{s, y-w}^{(N)} \Pi_{s^{\prime}, a-b}^{(N)} \Pi_{s^{\prime}, y^{\prime}-w^{\prime}}^{(N)} \boldsymbol{p}_{r-s}(a-y) \boldsymbol{p}_{r-s^{\prime}}\left(a-b-y^{\prime}\right) \\
\leq & {\left[A_{t} C_{t, 4, \varepsilon, \sigma} \operatorname{Lip}(\sigma)\right]^{2} \int_{0}^{r} \mathrm{~d} s \int_{\mathbb{R}^{d}} \mathrm{~d} y \int_{\mathbb{R}^{d}} f(\mathrm{~d} w) \int_{0}^{r} \mathrm{~d} s^{\prime} \int_{\mathbb{R}^{d}} \mathrm{~d} y^{\prime} \int_{\mathbb{R}^{d}} f\left(\mathrm{~d} w^{\prime}\right) } \\
& \times N^{-2 d} \Pi_{s, y-w}^{(N)} \Pi_{s^{\prime}, y^{\prime}-w^{\prime}}^{(N)} \boldsymbol{p}_{r-s}(a-y) \boldsymbol{p}_{r-s^{\prime}}\left(a-b-y^{\prime}\right) .
\end{aligned}
$$

This and the semigroup property of the heat kernel yield

$$
\begin{gathered}
\int_{\mathbb{R}^{d}} \mathrm{E}|\mathcal{A}(r, a) \mathcal{A}(r, a-b)| \mathrm{d} a \leq \frac{\left[A_{t} C_{t, 4, \varepsilon, \sigma} \operatorname{Lip}(\sigma)\right]^{2}}{N^{2 d}} \int_{0}^{r} \mathrm{~d} s \int_{\mathbb{R}^{d}} \mathrm{~d} y \int_{\mathbb{R}^{d}} f(\mathrm{~d} w) \int_{0}^{r} \mathrm{~d} s^{\prime} \int_{\mathbb{R}^{d}} \mathrm{~d} y^{\prime} \int_{\mathbb{R}^{d}} f\left(\mathrm{~d} w^{\prime}\right) \\
\times \Pi_{s, y-w}^{(N)} \Pi_{s^{\prime}, y^{\prime}-w^{\prime}}^{(N)} \boldsymbol{p}_{2 r-s-s^{\prime}}\left(y-b-y^{\prime}\right) .
\end{gathered}
$$

Apply the simple bound $\Pi_{s^{\prime}, y^{\prime}-w^{\prime}}^{(N)} \leq N^{-d}$ and first compute the $\mathrm{d} y^{\prime}$-integral to remove the term $\boldsymbol{p}_{s-s^{\prime}}\left(y-b-y^{\prime}\right)$, and then the dy-integral to see that

$$
\int_{\mathbb{R}^{d}} \mathrm{E}|\mathcal{A}(r, a) \mathcal{A}(r, a-b)| \mathrm{d} a \leq \frac{\left[r f\left(\mathbb{R}^{d}\right) A_{t} C_{t, 4, \varepsilon, \sigma} \operatorname{Lip}(\sigma)\right]^{2}}{N^{3 d}} .
$$

Plug this inequality into (6.7) to complete the proof. 
Proof of Theorem 2.4. Since $\operatorname{Var}(X+Y) \leq 2 \operatorname{Var}(X)+2 \operatorname{Var}(Y)$ for all random variables $X$ and $Y$, (6.5), and Lemmas 6.1 and 6.2 together yield

$$
\operatorname{Var}\left(K_{N}\right) \leq 2\left(L_{1}+L_{2}\right) t^{3} C_{t, 4, \varepsilon, \sigma}^{2} A_{t}^{2} N^{-d} .
$$

Because $|\sigma(z)| \leq|\sigma(0)|+\operatorname{Lip}(\sigma)|z|$ for all $z \in \mathbb{R}^{d}$, the large-deviations method of Foondun and Khoshnevisan [21] implies that $A_{t} \leq|\sigma(0)|+\operatorname{Lip}(\sigma) \sup _{s \in(0, t)}\|u(s, 0)\|_{4} \leq a \exp (b t)$ for all $t>0$, and for strictly positive numbers $a, b$ that depend only on $(f, \sigma)$. And Lemma 3.3 shows that $C_{t, 4, \varepsilon, \sigma} \leq c \exp (d t)$ for all $t>0$ and positive numbers $c$ and $d$ that depend only on $(f, \sigma)$. This proves immediately the existence of positive real numbers $\ell$ and $\lambda$ - depending only on $(f, \sigma)-$ such that

$$
\operatorname{Var}\left(K_{N}\right) \leq \frac{\ell \mathrm{e}^{2 \lambda t}}{N^{d}}
$$

It is easy to see that $d_{\mathrm{TV}}(\alpha X, \alpha Y)$ does not depend on the numerical value of $\alpha>0$ for all random variables $X$ and $Y$. Therefore, as long as $\mathbf{B}_{N, t}(g)>0,(2.1)$ implies that

$$
\begin{aligned}
& d_{\mathrm{TV}}\left(\frac{\mathcal{S}_{N, t}(g)}{\sqrt{\operatorname{Var}\left(\mathcal{S}_{N, t}(g)\right)}}, \mathrm{N}(0,1)\right)=d_{\mathrm{TV}}\left(\frac{N^{d / 2} \mathcal{S}_{N, t}(g)}{\sqrt{\mathbf{B}_{N, t}(g)}}, Z\right) \\
& =d_{\mathrm{TV}}\left(N^{d / 2} \mathcal{S}_{N, t}(g), \sqrt{\mathbf{B}_{N, t}(g)} Z\right) \leq \frac{2}{\mathbf{B}_{N, t}(g)} \mathrm{E}\left|K_{N}-\mathrm{E}\left(K_{N}\right)\right| ;
\end{aligned}
$$

see (4.13) for the last inequality. Apply the Cauchy-Schwarz inequality [see also (3.7)] and the preceding upper bound for $\operatorname{Var}\left(K_{N}\right)$ to complete the proof with $L=2 \sqrt{\ell}$, since $\lim _{N \rightarrow \infty} \mathbf{B}_{N, t}(g)=$ $\mathbf{B}_{t}(g)$ implies that indeed $\mathbf{B}_{N, t}(g)>0$ for all sufficiently large $N>0$.

\section{Parabolic Anderson Model: Proof of Theorem 2.5}

We begin by trying to mimic the proof of Theorem 2.4, except we do not assume that $g(v)=v$ for all $v \in \mathbb{R}^{d}$.

Recall $K_{N}$ from (4.6) and note that

$$
\operatorname{Var}\left(K_{N}\right)=N^{2 d} \operatorname{Var}\left(\int_{0}^{t} \mathrm{~d} s \int_{\mathbb{R}^{d}} \mathrm{~d} y D_{s, y} \mathcal{S}_{N, t}(g)\left(\mathrm{E}\left[D_{s, \bullet} \mathcal{S}_{N, t}(g) \mid \mathcal{F}_{s}\right] * f\right)(y)\right) .
$$

Therefore, we may take square roots and appeal to Minkowski's inequality [cf. (6.6)] in order to see that

$$
\sqrt{\operatorname{Var}\left(K_{N}\right)} \leq N^{d} \int_{0}^{t} \mathrm{~d} s \sqrt{\operatorname{Var}\left(\left\langle\mathrm{E}\left[D_{s, \bullet} \mathcal{S}_{N, t}(g) \mid \mathcal{F}_{s}\right], D_{s, \bullet} \mathcal{S}_{N, t}(g)\right\rangle_{\mathcal{H}_{0}}\right)} .
$$

We have seen already [see (4.2) and (6.2)] that

$$
D_{s, y} \mathcal{S}_{N, t}(g)=N^{-d} \int_{[0, N]^{d}} g^{\prime}(u(t, x)) D_{s, y} u(t, x) \mathrm{d} x, .
$$

where $D u(t, x)$ satisfies the linear stochastic integral equation,

$$
D_{s, y} u(t, x)=\boldsymbol{p}_{t-s}(x-y) u(s, y)+\int_{(s, t) \times \mathbb{R}^{d}} \boldsymbol{p}_{t-r}(x-z) D_{s, y} u(r, z) \eta(\mathrm{d} r \mathrm{~d} z) .
$$


See (1.8). It follows form these remarks that

$$
\begin{aligned}
D_{s, y} \mathcal{S}_{N, t}(g)=u(s, y) N^{-d} \int_{[0, N]^{d}} g^{\prime}(u(t, x)) \boldsymbol{p}_{t-s}(x-y) \mathrm{d} x \\
\quad+N^{-d} \int_{[0, N]^{d}} g^{\prime}(u(t, x))\left[\int_{(s, t) \times \mathbb{R}^{d}} \boldsymbol{p}_{t-r}(x-z) D_{s, y} u(r, z) \eta(\mathrm{d} r \mathrm{~d} z)\right] \mathrm{d} x .
\end{aligned}
$$

This is more or less (6.4), except that: (i) $g$ is not necessarily linear; (ii) $\sigma(z)=z$ for all $z \in \mathbb{R}$ here; and most significantly (iii) because of the presence of the non-anticipative term $g^{\prime}(u(t, x))$, we can no longer interchange the $\mathrm{d} x$-integral with the $\eta(\mathrm{d} r \mathrm{~d} z)$-stochastic integral. It is in fact because of item (iii) that we will need to adopt a different way to estimate $\operatorname{Var}\left(K_{N}\right)$ in the proof of Theorem 2.4. And at present the only other proof that we can construct works only when $g \in C^{2}(0, \infty)$ satisfies (2.12) and $\sigma(z)=z$ for all $z \in \mathbb{R}$, whence follow the conditions of Theorem 2.5.

According to (7.1) and the proof of Theorem 2.4, the key step of the argument is to find an efficient bound for

$$
\mathcal{V}(s)=\mathcal{V}_{N, t, g}(s):=\operatorname{Var}\left(\left\langle\mathrm{E}\left[D_{s, \bullet} \mathcal{S}_{N, t}(g) \mid \mathcal{F}_{s}\right], D_{s, \bullet} \mathcal{S}_{N, t}(g)\right\rangle_{\mathcal{H}_{0}}\right) \quad[0<s<t] .
$$

However, as was alluded to earlier in this section, the above estimation will need to involve other ideas than those that were used in the course of the proof of Theorem 2.4. Indeed, we plan to estimate $\mathcal{V}(s)$ by appealing to the Poincaré inequality (3.4). In order for this method to work, we will need to assume that $g \in C^{2}(0, \infty)$ satisfies $(2.12)$.

In the following, we abbreviate $\mathcal{S}_{N, t}(g)$ by $\mathcal{S}_{N}$ in order to simplify the typesetting. With this convention in mind, the Poincaré inequality yields

$$
\mathcal{V}(s) \leq \mathrm{E}\left(\left\|D\left\langle\mathrm{E}\left[D_{s, \bullet} \mathcal{S}_{N} \mid \mathcal{F}_{s}\right], D_{s, \bullet} \mathcal{S}_{N}\right\rangle_{\mathcal{H}_{0}}\right\|_{\mathcal{H}}^{2}\right)
$$

Now,

$$
\begin{aligned}
D_{r, z}\left\langle\mathrm{E}\left[D_{s, \bullet} \mathcal{S}_{N} \mid \mathcal{F}_{s}\right], D_{s, \bullet} \mathcal{S}_{N}\right\rangle_{\mathcal{H}_{0}}= & \int_{\mathbb{R}^{d}} \mathrm{~d} y \int_{\mathbb{R}^{d}} f\left(\mathrm{~d} y_{1}\right) \mathrm{E}\left[D_{s, y} \mathcal{S}_{N} \mid \mathcal{F}_{s}\right] D_{r, z} D_{s, y-y_{1}} \mathcal{S}_{N} \\
& +\int_{\mathbb{R}^{d}} \mathrm{~d} y \int_{\mathbb{R}^{d}} f\left(\mathrm{~d} y_{1}\right) D_{s, y} \mathcal{S}_{N} D_{r, z} \mathrm{E}\left[D_{s, y-y_{1}} \mathcal{S}_{N} \mid \mathcal{F}_{s}\right] \\
= & : \quad \Psi_{r, z}^{N, 1}+\Psi_{r, z}^{N, 2} .
\end{aligned}
$$

By Proposition 1.2.8 of Nualart [32], one can exchange the Malliavin derivative and the conditional expectation so that

$$
\Psi_{r, z}^{N, 2}=\int_{\mathbb{R}^{d}} \mathrm{~d} y \int_{\mathbb{R}^{d}} f\left(\mathrm{~d} y_{1}\right) D_{s, y} \mathcal{S}_{N} \mathrm{E}\left[D_{r, z} D_{s, y-y_{1}} \mathcal{S}_{N} \mid \mathcal{F}_{s}\right]
$$

Therefore, it follows that

$$
\mathcal{V}(s) \leq 2 \mathrm{E}\left(\left\|\Psi^{N, 1}\right\|_{\mathcal{H}}^{2}\right)+2 \mathrm{E}\left(\left\|\Psi^{N, 2}\right\|_{\mathcal{H}}^{2}\right)
$$

Both expectations can be estimated in the same way, and both estimates will yield the same upper bound. Therefore, we present only the details of the estimate for the first expectation. 
We can write

$$
\begin{aligned}
\mathrm{E}\left(\left\|\Psi^{N, 1}\right\|_{\mathcal{H}}^{2}\right)= & \int_{0}^{t} \mathrm{~d} r \int_{\mathbb{R}^{d}} \mathrm{~d} z \int_{\mathbb{R}^{d}} \mathrm{~d} y \int_{\mathbb{R}^{d}} \mathrm{~d} y^{\prime} \int_{\mathbb{R}^{d}} f\left(\mathrm{~d} z^{\prime}\right) \int_{\mathbb{R}^{d}} f\left(\mathrm{~d} y_{1}\right) \int_{\mathbb{R}^{d}} f\left(\mathrm{~d} y_{1}^{\prime}\right) \\
& \times \mathrm{E}\left[\mathrm{E}\left[D_{s, y} \mathcal{S}_{N} \mid \mathcal{F}_{s}\right] D_{r, z} D_{s, y-y 1} \mathcal{S}_{N}\right. \\
& \left.\times \mathrm{E}\left[D_{s, y^{\prime}} \mathcal{S}_{N} \mid \mathcal{F}_{s}\right] D_{r, z-z^{\prime}} D_{s, y^{\prime}-y_{1}^{\prime}} \mathcal{S}_{N}\right] .
\end{aligned}
$$

Then an application of Hölder inequality shows that

$$
\begin{aligned}
\mathrm{E}\left(\left\|\Psi^{N, 1}\right\|_{\mathcal{H}}^{2}\right) \leq & \int_{0}^{t} \mathrm{~d} r \int_{\mathbb{R}^{d}} \mathrm{~d} z \int_{\mathbb{R}^{d}} \mathrm{~d} y \int_{\mathbb{R}^{d}} \mathrm{~d} y^{\prime} \int_{\mathbb{R}^{d}} f\left(\mathrm{~d} z^{\prime}\right) \int_{\mathbb{R}^{d}} f\left(\mathrm{~d} y_{1}\right) \int_{\mathbb{R}^{d}} f\left(\mathrm{~d} y_{1}^{\prime}\right) \\
& \times\left\|D_{s, y} \mathcal{S}_{N}\right\|_{4}\left\|D_{r, z} D_{s, y-y_{1}} \mathcal{S}_{N}\right\|_{4}\left\|D_{s, y^{\prime}} \mathcal{S}_{N}\right\|_{4}\left\|D_{r, z-z^{\prime}} D_{s, y^{\prime}-y_{1}^{\prime}} \mathcal{S}_{N}\right\|_{4}
\end{aligned}
$$

According to Lemma 3.3 and Hölder's inequality,

$$
\left\|D_{s, y} \mathcal{S}_{N}\right\|_{4} \leq\left\|g^{\prime}(u(t, 0))\right\|_{k} \frac{1}{N^{d}} \int_{[0, N]^{d}}\left\|D_{s, y} u(t, x)\right\|_{\frac{4 k}{k-4}} \mathrm{~d} x \leq C_{t, \frac{4 k}{k-4}, \varepsilon}\left\|g^{\prime}(u(t, 0))\right\|_{k} \Pi_{s, y}^{(N)},
$$

where $\varepsilon \in(0,1)$ is arbitrary and $\Pi^{(N)}$ was defined in (6.3). Next we apply (4.2) in order to see that

$$
\begin{aligned}
D_{r, z} D_{s, y} \mathcal{S}_{N}= & N^{-d} \int_{[0, N]^{d}} g^{\prime}(u(t, x)) D_{r, z} D_{s, y} u(t, x) \mathrm{d} x \\
& +N^{-d} \int_{[0, N]^{d}} g^{\prime \prime}(u(t, x)) D_{r, z} u(t, x) D_{s, y} u(t, x) \mathrm{d} x .
\end{aligned}
$$

Therefore, Proposition 3.4, Lemma 3.3, and the notation for $\Pi^{(N)}$ in (6.3) together yield

$$
\begin{aligned}
\left\|D_{r, z} D_{s, y} \mathcal{S}_{N}\right\|_{4} \leq & C_{t, \frac{4 k}{k-4}, \varepsilon}^{*}\left\|g^{\prime}(u(t, 0))\right\|_{k}\left[\boldsymbol{p}_{s-r}(y-z) \mathbf{1}_{\{r \leq s\}} \Pi_{s, y}^{(N)}+\boldsymbol{p}_{r-s}(z-y) \mathbf{1}_{\{s \leq r\}} \Pi_{r, z}^{(N)}\right] \\
& +\frac{C_{t, \frac{8 k}{k-4}, \varepsilon, \sigma}^{2}\left\|g^{\prime \prime}(u(t, 0))\right\|_{k}}{N^{d}} \int_{[0, N]^{d}} \boldsymbol{p}_{t-r}(x-z) \boldsymbol{p}_{t-s}(x-y) \mathrm{d} x \\
\leq & \frac{C_{t, \frac{4 k}{k-4}, \varepsilon}^{*}\left\|g^{\prime}(u(t, 0))\right\|_{k}}{N^{d}} \boldsymbol{p}_{|s-r|}(y-z)+\frac{C_{t, \frac{8 k}{k-4}, \varepsilon, \sigma}^{2}\left\|g^{\prime \prime}(u(t, 0))\right\|_{k}}{N^{d}} \boldsymbol{p}_{2 t-r-s}(y-z),
\end{aligned}
$$

owing to the semigroup property of the heat kernel and Rademacher's theorem (Remark 4.1), where the constant $C_{t, 4, \epsilon}^{*}$ is defined in (3.12). Thus,

$$
\begin{aligned}
\mathrm{E}\left(\left\|\Psi^{N, 1}\right\|_{\mathcal{H}}^{2}\right) \leq & C_{1}\left\|g^{\prime}(u(t, 0))\right\|_{k}^{2} \\
& \times \int_{0}^{t} \mathrm{~d} r \int_{\mathbb{R}^{d}} \mathrm{~d} z \int_{\mathbb{R}^{d}} \mathrm{~d} y \int_{\mathbb{R}^{d}} \mathrm{~d} y^{\prime} \int_{\mathbb{R}^{d}} f\left(\mathrm{~d} z^{\prime}\right) \int_{\mathbb{R}^{d}} f\left(\mathrm{~d} y_{1}\right) \int_{\mathbb{R}^{d}} f\left(\mathrm{~d} y_{1}^{\prime}\right) \Pi_{s, y}^{(N)} \Pi_{s, y^{\prime}}^{(N)} \\
& \times\left(\frac{C_{2}\left\|g^{\prime}(u(t, 0))\right\|_{k}}{N^{d}} \boldsymbol{p}_{|s-r|}\left(y-y_{1}-z\right)+\frac{C_{3}\left\|g^{\prime \prime}(u(t, 0))\right\|_{k}}{N^{d}} \boldsymbol{p}_{2 t-r-s}\left(y-y_{1}-z\right)\right) \\
& \times\left(\frac{C_{2}\left\|g^{\prime}(u(t, 0))\right\|_{k}}{N^{d}} \boldsymbol{p}_{|s-r|}\left(y^{\prime}-y_{1}^{\prime}-z+z^{\prime}\right)+\frac{C_{3}\left\|g^{\prime \prime}(u(t, 0))\right\|_{k}}{N^{d}} \boldsymbol{p}_{2 t-r-s}\left(y^{\prime}-y_{1}^{\prime}-z+z^{\prime}\right)\right),
\end{aligned}
$$


where $C_{1}=C_{t, \frac{4 k}{k-4}, \varepsilon}^{2}, C_{2}=C_{t, \frac{4 k}{k-4}, \varepsilon}^{*}$ and $C_{3}=C_{t, \frac{8 k}{k-4}, \varepsilon, \sigma}^{2}$. We apply the bound $\Pi_{s, y^{\prime}}^{(N)} \leq N^{-d}$ and integrate [first $\mathrm{d} y^{\prime}$, then $\mathrm{d} z$, and then finally $\mathrm{d} y$, and in this order], using also the inequality $\int_{\mathbb{R}^{d}} \Pi_{s, y}^{(N)} \mathrm{d} y \leq 1$, in order to obtain

$$
\mathrm{E}\left(\left\|\Psi^{N, 1}\right\|_{\mathcal{H}}^{2}\right) \leq C_{4}\left[f\left(\mathbb{R}^{d}\right)\right]^{3} t N^{-3 d}
$$

where

$$
C_{4}=C_{1}\left\|g^{\prime}(u(t, 0))\right\|_{k}^{2}\left[C_{2}\left\|g^{\prime}(u(t, 0))\right\|_{k}+C_{3}\left\|g^{\prime \prime}(u(t, 0))\right\|_{k}\right]^{2} .
$$

The very same method applies and yields the same upper bound for $\mathrm{E}\left(\left\|\Psi^{N, 2}\right\|_{\mathcal{H}}^{2}\right)$. Therefore, we may deduce from (7.3) and (7.4) that $\mathcal{V}(s) \leq 4 C_{4} t N^{-3 d}\left[f\left(\mathbb{R}^{d}\right)\right]^{3}$. We plug this estimate into (7.1) to obtain the following:

$$
\sqrt{\operatorname{Var}\left(K_{N}\right)} \leq N^{d} \int_{0}^{t} \sqrt{\mathcal{V}(s)} \mathrm{d} s \leq 2 C_{4}^{1 / 2} t^{3 / 2}\left[f\left(\mathbb{R}^{d}\right)\right]^{3 / 2} N^{-d / 2}
$$

This is the replacement of the inequality (6.8) in the present setting of the parabolic Anderson model; the remainder of the proof of Theorem 2.4 goes through unhindered to complete the proof of Theorem 2.5.

\section{A Association for parabolic SPDEs}

We conclude the paper by showing how we can adapt the ideas of this paper to also document a result about the association of the solution to (1.1). First, let us recall the following.

Definition A.1 (Esary, Proschan, and Walkup [20]). A random vector $X:=\left(X_{1}, \ldots, X_{n}\right)$ is said to be associated if

$$
\operatorname{Cov}\left[h_{1}(X), h_{2}(X)\right] \geq 0,
$$

for every pair of functions $h_{1}, h_{2}: \mathbb{R}^{n} \rightarrow \mathbb{R}$ that are nondecreasing in every coordinate and satisfy $h_{1}(X), h_{2}(X) \in L^{2}(\Omega)$.

It is easy to see that $h_{1}$ and $h_{2}$ could instead be assumed to be nonincreasing in every coordinate: One simply replaces $\left(h_{1}, h_{2}\right)$ by $\left(-h_{1},-h_{2}\right)$. Other simple variations abound. Esary, Proschan, and Walkup [20] prove the more interesting result that $X$ is associated iff (A.1) holds for all $h_{1}, h_{2} \in C_{b}\left(\mathbb{R}^{n}\right)$ that are nondecreasing coordinatewise. ${ }^{7}$ This and the dominated convergence theorem together imply the following simple criterion for association; see also Pitt [35].

Lemma A.2. $X=\left(X_{1}, \ldots, X_{n}\right)$ is associated iff (A.1) holds for all $h_{1}, h_{2} \in C_{c}^{\infty}\left(\mathbb{R}^{n}\right)$ such that $\partial_{i} h_{j} \geq 0$ for every $i=1,2$ and $j=1, \ldots, n .^{8}$

The notion of association has a natural extension to random processes. Here is one.

Definition A.3. A random field $\Phi=\{\Phi(t, x)\}_{t>0, x \in \mathbb{R}^{d}}$ is associated if $\left(\Phi\left(t_{1}, x_{1}\right), \ldots, \Phi\left(t_{n}, x_{n}\right)\right)$ is associated for every $t_{1}, \ldots, t_{n}>0$ and $x_{1}, \ldots, x_{n} \in \mathbb{R}^{d}$.

The following is the principal result of this appendix.

\footnotetext{
${ }^{7}$ Recall that $C_{b}\left(\mathbb{R}^{n}\right)$ denotes the collection of all real functions on $\mathbb{R}^{n}$ that are bounded and continuous.

${ }^{8}$ Recall that the subscript " $c$ " in $C_{c}^{\infty}$ refers to functions of compact support.
} 
Theorem A.4. Let $u$ be the solution to (1.1) with $u(0, \cdot)$ being a nonnegative measure such that $\left(u(0, \cdot) * \boldsymbol{p}_{t}\right)<\infty$ for all $t>0$. Under Dalang's condition (1.5), if $\sigma \circ u$ a.s. does not change signs; that is,

$$
\mathrm{P}\{\sigma(u(t, x)) \sigma(u(s, y)) \geq 0\}=1 \quad \text { for every } s, t>0 \text { and } x, y \in \mathbb{R}^{d},
$$

then, $u$ is associated.

Theorem A.4 reduces to simpler results in two important special cases that we present in the following two examples.

Example A.5. Condition (A.2) holds tautologically when $\sigma$ is a constant, say $\sigma \equiv \sigma_{0} \in \mathbb{R}$. In that case, it is not hard to see that the solution to (1.1) is a Gaussian random field with covariance function,

$$
\operatorname{Cov}\left[u(t, x), u\left(t^{\prime}, x^{\prime}\right)\right]=\sigma_{0}^{2} \int_{0}^{t}\left(\boldsymbol{p}_{2 s} * f\right)\left(x-x^{\prime}\right) \mathrm{d} s .
$$

Because this quantity is $\geq 0$ for every $t, t^{\prime}>0$ and $x, x^{\prime} \in \mathbb{R}^{d}$, Pitt's characterization of Gaussian random vectors [35] immediately implies the association of $u$ when $\sigma$ is a constant.

Example A.6. If $\sigma(u)=u, d=1$, and $f=\delta_{0}$, then the sign condition (A.2) holds simply because $u(t, x) \geq 0$ a.s. In this case, Corwin and Quastel [14, Proposition 1] have observed that $u$ is associated; see also Corwin and Ghosal [13, Proposition 1.9]. Strictly speaking, the statements of the latter two results are weaker that the association of $u$. But the proof of Proposition 1.9 of Corwin and Quastel (ibid.) goes through unhindered to imply association. Indeed, Corwin and Quastel note the said association from the well-known fact that all exclusion processes are associated (the FKG inequality for i.i.d. random variables).

The preceding examples are proved using two different "direct hands-on" methods. The general case is quite a bit more involved. In order to avoid writing a lengthy proof, we merely outline the proof, with detailed pointers to the requisite literature and preceding results of the present paper.

Outline of the proof of Theorem A.4. By considering $-\sigma$ in place of $\sigma$ if need be, we may - and will - assume without loss in generality that

$$
\sigma(u(t, x)) \geq 0 \quad \text { a.s. for every }(t, x) \in(0, \infty) \times \mathbb{R}^{d} .
$$

We begin by rehashing through the proof of Theorem 3.2, and recall that the Malliavin derivative of $u(t, x)$ a.s. solves

$$
D_{s, z} u(t, x)=\boldsymbol{p}_{t-s}(x-z) \sigma(u(s, z))+\int_{(s, t) \times \mathbb{R}^{d}} \boldsymbol{p}_{t-r}(x-y) \sigma^{\prime}(u(r, y)) D_{s, z} u(r, y) \eta(\mathrm{d} r \mathrm{~d} y),
$$

for almost all $(s, z) \in(0, t) \times \mathbb{R}^{d}$. And, of course, $D_{s, z} u(t, x)=0$ a.s. for a.e. $(s, z) \in(t, \infty) \times \mathbb{R}^{d}$. Note that the right-hand side of (A.3) is in fact a modification of $D_{s, z} u(t, x)$. Therefore, we can and will without loss of generality - redefine $D_{s, z} u(t, x)$ so that (A.3) is an almost-sure identity for every $(s, z) \in(0, t) \times \mathbb{R}^{d}$, and that $D_{s, z} u(t, x):=0$ whenever $s \geq t$. It is not hard to deduce from (A.3), and arguments involving continuity in probability, that the use of this particular modification does not change the law of the Malliavin derivative. However, it makes the following discussion simpler as we can avoid worrying about null sets.

We aim to prove that, under Dalang's condition (1.5) alone,

$$
D_{s, z} u(t, x) \geq 0 \quad \text { a.s. for every }(t, x) \in(0, \infty) \times \mathbb{R}^{d} .
$$


Once this is proved, one can appeal to the Clark-Ocone formula (3.3) to conclude the theorem; see the proof of Theorem 4.6 for a similar argument.

Choose and fix $(s, z) \in(0, \infty) \times \mathbb{R}^{d}$, and consider a space-time random field $\Phi_{s, z}$ defined via

$$
\Phi_{s, z}(t, x):=D_{s, z} u(s+t, z+x) \quad \text { for all } t \geq 0 \text { and } x \in \mathbb{R}^{d} .
$$

Our claim (A.4) is equivalent to the a.s.-nonnegativity of $\Phi_{s, z}(t, x)$.

Observe (similarly to what we did in the proof of Theorem 3.2), that $\Phi_{s, z}$ solves

$$
\Phi_{s, z}(t, x)=\boldsymbol{p}_{t}(z+x) \sigma(u(s, z))+\int_{(0, t) \times \mathbb{R}^{d}} \boldsymbol{p}_{t-r}(x-y) H_{s, z}(t, y) \Phi_{s, z}(r, y) \eta_{s, z}(\mathrm{~d} r \mathrm{~d} y),
$$

for $(t, x) \in(0, \infty) \times \mathbb{R}^{d}$, where $\eta_{s, z}$ is the same shifted Gaussian noise that arose in the course of the proof of Theorem 3.2, and

$$
H_{s, z}(t, y)=\sigma^{\prime}(u(s+t, z+y)) \text {, }
$$

also as in the proof of Theorem 3.2. In other words, conditional on the sigma-algebra $\mathcal{F}_{s}$, the random field $\Phi_{s, z}$ solves the SPDE,

$$
\partial_{t} \Phi_{s, z}=\frac{1}{2} \Delta \Phi_{s, z}+\Phi_{s, z} H_{s, z} \eta_{s, z} \quad \text { subject to } \Phi_{s, z}(0, x)=\sigma(u(s, z)) \delta_{0}(x) .
$$

This is a standard Walsh-type SPDE. In order to understand why this is the case, note first that $u(s, z)$ is $\mathcal{F}_{s}$-measurable and $\eta_{s, z}$ is independent of $\mathcal{F}_{s}$; see also the proof of Lemma 3.2. In order to complete the assertion about (A.5) being a standard Walsh-type SPDE we note that $M_{t}(A):=\int_{(0, t) \times A} H_{s, z}(r, y) \eta_{s, z}(\mathrm{~d} r \mathrm{~d} y)$ defines a martingale measure in the sense of Walsh. Hence, we view (A.5) conditionally on $\mathcal{F}_{s}$ to see that it is a reformulation of the Walsh SPDE,

$$
\partial_{t} \Phi_{s, z}=\frac{1}{2} \Delta \Phi_{s, z}+\Phi_{s, z} \dot{M}_{t}(x) \quad \text { subject to } \quad \Phi_{s, z}(0, x)=\sigma(u(s, z)) \delta_{0}(x),
$$

also viewed conditionally on $\mathcal{F}_{s}$. In fact, we can interpret (A.5) as a parabolic Anderson model with respect to the martingale measure $M$. Because of (1.11), and since $\sigma \in \operatorname{Lip,~} M$ is in fact a worthy martingale measure because $H_{s, z}$ is bounded; see Walsh [38].

For every $\varepsilon \in(0,1)$ let $\eta_{s, z}^{\varepsilon}$ denote the Gaussian noise

$$
\eta_{s, z}^{\varepsilon}(t, x) \mathrm{d} t:=\int_{\mathbb{R}^{d}} \boldsymbol{p}_{\varepsilon}(x-y) \eta_{s, z}(\mathrm{~d} t \mathrm{~d} y) .
$$

The spatial correlation measure $f_{\varepsilon}$ of $\eta_{s, z}^{\varepsilon}$ is in fact a function [not just a measure] and is given by $f_{\varepsilon}=\boldsymbol{p}_{2 \varepsilon} * f$. Because $f$ is a finite measure, it follows that $f_{\varepsilon}$ satisfies the extended Dalang condition (1.6) with $\alpha=1$. Let $\Phi_{s, z}^{\varepsilon}$ denote the unique solution to the SPDE,

$$
\partial_{t} \Phi_{s, z}^{\varepsilon}=\frac{1}{2} \Delta \Phi_{s, z}^{\varepsilon}+\left(\sigma^{\prime} \circ u\right) \Phi_{s, z}^{\varepsilon} \eta_{s, z}^{\varepsilon} \quad \text { subject to } \quad \Phi_{s, z}(0, x)=\sigma(u(s, z)) \delta_{0}(x) .
$$

This is the same SPDE as (A.5), but with respect to the mollified version $\eta_{s, z}^{\varepsilon}$ of the Gaussian noise $\eta_{s, z}$ in place of $\eta_{s, z}$. Because $f_{\varepsilon}$ satisfies (1.6) for some $\alpha>0$, Theorem 1.6 of Chen and Huang [5] [applied conditionally on $\mathcal{F}_{s}$ ] implies that, if $H_{s, z}$ were a constant [that is, $\sigma(u) \propto u$ ], then $\Phi_{s, z}^{\varepsilon}(t, x)>0$ a.s. for every $t>0$ and $x \in \mathbb{R}^{d}$. Careful scrutiny of the proof of Theorem 1.6 of [5], using the boundedness of $H_{s, z}$, yields that $\Phi_{s, z}^{\varepsilon}>0$ a.s. in all cases; see also Theorem 1.8 of Chen and Huang [6]. Finally, we let $\varepsilon \downarrow 0$ and appeal to Theorem 1.9 of Chen and Huang [5] to see that $\lim _{\varepsilon \rightarrow 0} \Phi_{s, z}^{\varepsilon}(t, x)=\Phi_{s, z}(t, x)$ in $L^{2}(\Omega)$ for all $(t, x) \in(0, \infty) \times \mathbb{R}^{d}$. These facts, together imply $\Phi_{s, z}(t, x) \geq 0$ a.s. for all $(t, x) \in(0, \infty) \times \mathbb{R}^{d}$, which is another way to write (A.4). This concludes the proof. 


\section{References}

[1] Bradley, Richard C. (2007). Introduction to Strong Mixing Conditions, Vol. 1. Kendrick Press, Heber City, Utah.

[2] Burkholder, D. L., Davis, B. J., and Gundy, R. F. (1972). Integral inequalities for convex functions of operators on martingales. In: Proceedings of the Sixth Berkeley Symposium on Mathematical Statistics and Probability II, 223-240, University of California Press, Berkeley, California.

[3] Carlen, Eric and Paul Krée (1991). $L^{p}$ estimates on iterated stochastic integrals. Ann. Probab. 19(1) $354-368$.

[4] Chen, Le, Yaozhong Hu and David Nualart (2016) Regularity and strict positivity of densities for the nonlinear stochastic heat equation. arXiv:1611.03909. To appear in Mem. Amer. Math. Soc.

[5] Chen, Le, Jingyu Huang (2019). Comparison principle for stochastic heat equation on $\mathbb{R}^{d}$. Ann. Probab. 47, no. 2, 98-1035.

[6] Chen, Le, Jingyu Huang (2019). Regularity and strict positivity of densities for the stochastic heat equation on $\mathbb{R}^{d}$. Preprint available at https://arxiv.org/abs/1902.02382.

[7] Chen, Le, Davar Khoshnevisan, David Nualart, and Fei Pu (2019). Spatial ergodicity for SPDEs via Poincaré-type inequalities. Preprint available at https://arxiv.org/abs/1907.11553.

[8] Chen, Le, Davar Khoshnevisan, David Nualart, and Fei Pu (2019). Poincaré inequality, and central limit theorems for parabolic stochastic partial differential equations. Preprint available at https://arxiv.org/abs/1912.01482.

[9] Chen, Le, Davar Khoshnevisan, David Nualart, and Fei Pu (2020). A CLT for dependent random variables, with an application to an infinite system of interacting diffusion processes. Preprint available at https://arxiv.org/abs/2005.05827.

[10] Chen, Le and Kunwoo Kim (2019). Nonlinear stochastic heat equation driven by spatially colored noise: moments and intermittency. Acta Math. Sci. (English Ed.) 39, no. 3: 645-668.

[11] Daniel Conus, Mathew Joseph and Davar Khshnevisan (2012). Correlation-length bounds, and estimates for intermittent islands in parabolic SPDEs. Electron. J. Probab. 17, no. 102, 15pp.

[12] Cover, Thomas M. and Joy A. Thomas (2006). Elements of Information Theory, 2nd edition, WilleyInterscience.

[13] Ivan Corwin and Promit Ghosal (2018). KPZ equation tails for general initial data. Preprint available at https://arxiv.org/abs/1810.07129.

[14] Ivan Corwin and Jeremy Quastel (2013). Crossover distributions at the edge of the rarefaction fan. Ann. Probab. 41, no. 3A, 1243-1314

[15] Dalang, Robert C. (1999). Extending the martingale measure stochastic integral with applications to spatially homogeneous s.p.d.e.'s. Electron. J. Probab. 4(6), 29 pp.

[16] Deuschel, Jean-Dominique (1988). Central limit theorem for an infinite lattice system of interacting diffusion processes (1988). Ann. Probab. 16(2) 700-716.

[17] Dym, H. and H. P. McKean (1976). Gaussian Processes, Function Theory, and the Inverse Spectral Problem. Academic Press. New York.

[18] Federer, Herbert (1969). Geometric Measure Theory. Die Grundlehren der mathematischen Wissenschaften 153 Springer-Verlag, Berlin-Heidelberg-New York. 
[19] Doob, J. L. (1990). Stochastic Processes. Reprint of the 1953 original. John Wiley \& Sons, Inc., New York, viii+654.

[20] Esary, J. D., F. Proschan, and D. W. Walkup (1967). Association of random variables with applications. Ann. Math. Statist. 38(5) 1466-1474.

[21] Foondun, Mohammud and Davar Khoshnevisan (2013). On the stochastic heat equation with spatiallycolored random forcing. Trans. Amer. Math. Soc. 365(1) 409-458.

[22] Helson, Henry and Donald Sarason (1967). Past and future. Math. Scand. 21 5-16 (1968).

[23] Huang, Jingyu, Khoa Lê, and David Nualart (2017). Large time asymptotics for the parabolic Anderson model driven by space and time correlated noise. Stoch. Partial Differ. Equ. Anal. Comput. 5(4) 614-651.

[24] Huang, Jingyu, David Nualart, and Lauri Viitasaari (2018). A central limit theorem for the stochastic heat equation. Preprint available at https://arxiv.org/abs/1810.09492.

[25] Jingyu Hunag, David Nualart, Lauri Viitasaari, and Guangqu Zheng (2020). Gaussian fluctuations for the stochastic heat equation with colored noise. Stoch PDE: Anal Comp 8 402-421.

[26] Kardar, K., Parisi, G., Zhang, Y. Z. (1986). Dynamic scaling of growing interfaces. Phys. Rev. Lett. 56 889-892.

[27] Maruyama, Gisirō (1949). The harmonic analysis of stationary stochastic processes. Mem. Faculty Sci. Kyushu Univ. Ser. A. 4 45-106.

[28] Newman, Charles M. (1983). A general central limit theorem for FKG systems. Comm. Math. Phys. $9175-80$.

[29] Newman, C. M. and A. L. Wright (1981). An invariance principle for certain dependent sequences. Ann. Probab. 9(6) 671-675.

[30] Nourdin, Ivan and Peccati, Giovanni (2009). Stein's method on wiener chaos. Probab. Theory Related Fields, 1451 75-118.

[31] Nourdin, Ivan and Peccati, Giovanni (2012). Normal approximations with Malliavin calculus. From Stein's method to universality. Cambridge University Press, Cambridge, UK..

[32] Nualart, David (2006). The Malliavin Calculus and Related Topics. Springer, New York.

[33] Nualart, David and Nualart, Eulàlia (2018). An introduction to Malliavin calculus. Cambridge University Press, Cambridge, UK.

[34] Nualart, David and Zheng, Guangqu (2020). Averaging Gaussian functionals. Electron J. Probab. 25, paper no. 48, 54 pp.

[35] Pitt, Loren (1982). Positively correlated normal variables are associated. Ann. Probab. 10(2) 496-499.

[36] Prakasa Rao, B. L. S. (2012). Associated Sequences, Demimartingales and Nonparametric Inference. Birkhäuser/Springer, Basel, xii+272 pp.

[37] Sanz-Solé, Marta and Mònica Sarrà (2002). Hölder continuity for the stochastic heat equation with spatially correlated noise. In: Seminar on Stochastic Analysis, Random Fields and Applications, III (Ascona, 1999), 259-268, Progr. Probab., 52, Birkhäuser, Basel.

[38] Walsh, John B. (1986). An Introduction to Stochastic Partial Differential Equations. Ècole d'été de probabilités de Saint-Flour, XIV-1984, 265-439, In: Lecture Notes in Math. 1180, Springer, Berlin. 